\title{
Risk characterisation of chemicals in food and diet
}

Citation for published version (APA):

Renwick, A. G., Barlow, S. M., Hertz-Picciotto, I., Boobis, A. R., Dybing, E., Ädler, L., Eisenbrand, G., Greig, J. B., Kleiner, J., Lambe, J., Müller, D. J., Smith, M. R., Tritscher, A., Tuijtelaars, S., van den Brandt, P. A., Walker, R., \& Kroes, R. (2003). Risk characterisation of chemicals in food and diet. Food and Chemical Toxicology, 41(9), 1211-1271. https://doi.org/10.1016/S0278-6915(03)00064-4

Document status and date:

Published: 01/01/2003

DOI:

10.1016/S0278-6915(03)00064-4

Document Version:

Publisher's PDF, also known as Version of record

\section{Please check the document version of this publication:}

- A submitted manuscript is the version of the article upon submission and before peer-review. There can be important differences between the submitted version and the official published version of record.

People interested in the research are advised to contact the author for the final version of the publication, or visit the DOI to the publisher's website.

- The final author version and the galley proof are versions of the publication after peer review.

- The final published version features the final layout of the paper including the volume, issue and page numbers.

Link to publication

\footnotetext{
General rights rights.

- You may freely distribute the URL identifying the publication in the public portal. please follow below link for the End User Agreement:

www.umlib.nl/taverne-license

Take down policy

If you believe that this document breaches copyright please contact us at:

repository@maastrichtuniversity.nl

providing details and we will investigate your claim.
}

Copyright and moral rights for the publications made accessible in the public portal are retained by the authors and/or other copyright owners and it is a condition of accessing publications that users recognise and abide by the legal requirements associated with these

- Users may download and print one copy of any publication from the public portal for the purpose of private study or research.

- You may not further distribute the material or use it for any profit-making activity or commercial gain

If the publication is distributed under the terms of Article $25 \mathrm{fa}$ of the Dutch Copyright Act, indicated by the "Taverne" license above, 


\title{
Risk characterisation of chemicals in food and diet ${ }^{\text {is }}$
}

\author{
A.G. Renwick ${ }^{\mathrm{a}}$, S.M. Barlow ${ }^{\mathrm{b}}$ I. Hertz-Picciotto ${ }^{\mathrm{c}}$, A.R. Boobis ${ }^{\mathrm{d}}$, E. Dybing ${ }^{\mathrm{e}}$, L. Edler $^{\mathrm{f}}$, \\ G. Eisenbrand ${ }^{\mathrm{g}}$, J.B. Greig ${ }^{\mathrm{h}}$, J. Kleiner ${ }^{\mathrm{i}, *}$, J. Lambe ${ }^{\mathrm{j}}$, D.J.G. Müller ${ }^{\mathrm{k}}$, M.R. Smith ${ }^{1}$, \\ A. Tritscher ${ }^{\mathrm{m}}$, S. Tuijtelaarsi ${ }^{\mathrm{i}}$, P.A. van den Brandt ${ }^{\mathrm{n}}$, R. Walker ${ }^{\mathrm{o}}$, R. Kroes ${ }^{\mathrm{p}}{ }^{\dagger \dagger}$ \\ ${ }^{a}$ University of Southampton, Clinical Pharmacology Group, School of Medicine, Biomedical Sciences Building, \\ Bassett Crescent East, Southampton SO16 7PX, UK \\ ${ }^{\mathrm{b}} \mathrm{MRC}$ Institute for Environment and Health, University of Leicester, 94 Regent Road, Leicester LE1 7DD, UK \\ ${ }^{\mathrm{c}}$ University of California, Davis, Department of Epidemiology and Preventive Medicine, TB-168, One Shields Avenue, Davis, CA 95616, USA \\ ${ }^{\mathrm{d}}$ Imperial College, Section on Clinical Pharmacology, Division of Medicine, Hammersmith Campus, Ducane Road, London W12 0NN, UK \\ ${ }^{\mathrm{e}}$ Norwegian Institute of Public Health, Division of Environmental Medicine, PO Box 4404 Nydalen, N-0403 Oslo, Norway \\ ${ }^{\mathrm{f}}$ Deutsches Krebsforschungszentrum, (German Cancer Research Center), Biostatistics Unit C060, PO Box 1019 49, D-69009 Heidelberg, Germany \\ ${ }^{\mathrm{g}}$ University of Kaiserslautern, Department of Chemistry, Food Chemistry \& Environmental Toxicology, PO Box 3049, D-67653 Kaiserslautern, Germany \\ ${ }^{\mathrm{h}}$ Independent Consultant, $U K$ \\ ${ }^{\text {i } I L S I ~ E u r o p e, ~ A v e n u e ~ E . ~ M o u n i e r ~} 83$ Box 6, B-1200 Brussels, Belgium \\ ${ }^{\mathrm{j} I n s t i t u t e ~ o f ~ E u r o p e a n ~ F o o d ~ S t u d i e s, ~ B i o t e c h n o l o g y ~ I n s t i t u t e, ~ T r i n i t y ~ C o l l e g e, ~ I R L-D u b l i n ~ 2, ~ I r e l a n d ~}$ \\ ${ }^{\mathrm{k}}$ Procter \& Gamble Service GmbH, Sulzbacher Str. 40, D-65824 Schwalbach am Taunus, Germany \\ ${ }^{1}$ Unilever Health Institute, Unilever Research Vlaardingen, Olivier van Noortlaan 120, 3133 AT Vlaardingen, The Netherlands \\ ${ }^{\mathrm{m}}$ Nestlé Research Centre, Vers-Chez-Les-Blanc, PO Box 44, CH-1000 Lausanne 26, Switzerland \\ ${ }^{\mathrm{n}}$ Maastricht University, Department of Epidemiology, P.O. Box 616, NL-6200 MD Maastricht, The Netherlands \\ ${ }^{\circ}$ University of Surrey, School of Biomedical and Life Sciences, Guildford GU2 $5 X H$, Surrey, UK \\ ${ }^{\mathrm{p} U t r e c h t ~ U n i v e r s i t y, ~ I n s t i t u t e ~ f o r ~ R i s k ~ A s s e s s m e n t ~ S c i e n c e s ~(I R A S), ~ F a c u l t y ~ o f ~ V e t e r i n a r y ~ M e d i c i n e, ~ Y a l e l a a n ~ 2, ~ P O ~ B o x ~} 80176$,
} NL-3508 TD Utrecht, The Netherlands

Accepted 2 February 2003

\section{Contents}

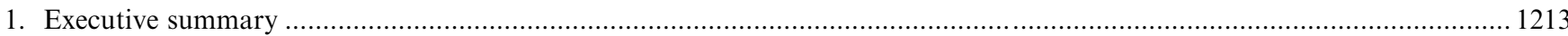

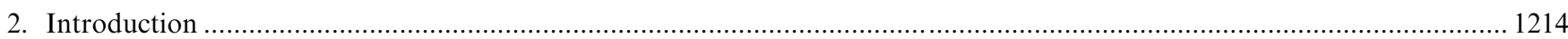

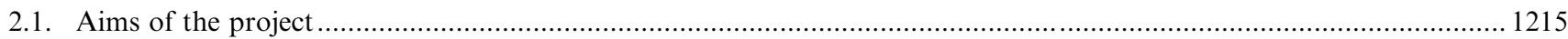

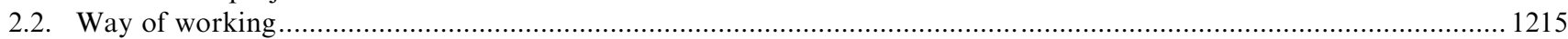

2.3. Risk characterisation - the last step in the risk assessment process .......................................................... 1215

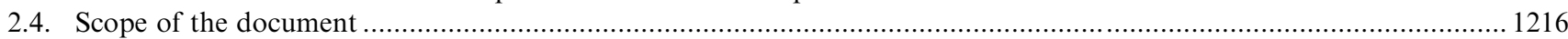

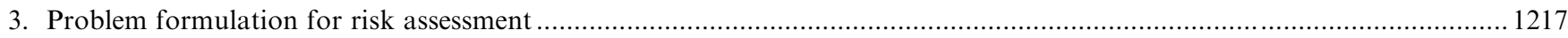

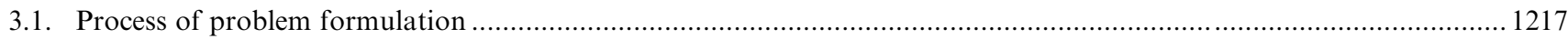

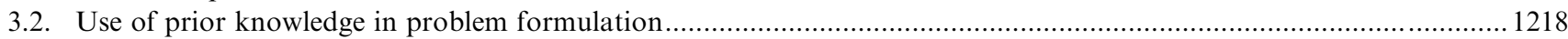

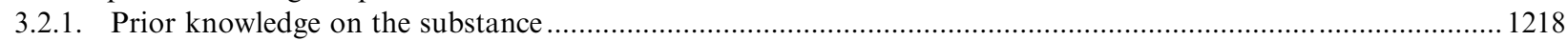

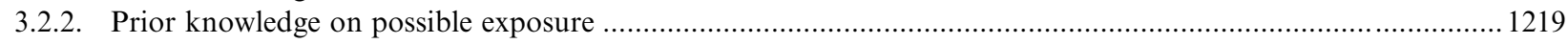

3.2.3. Prior knowledge on possible biological effect(s) of the substance .................................................... 1219

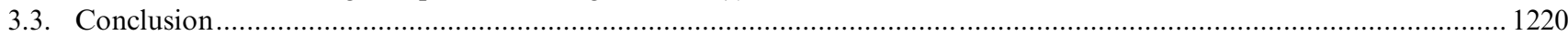

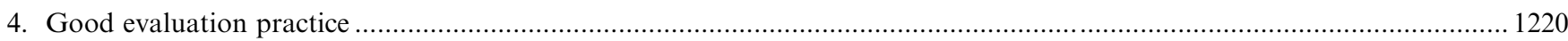

is The views expressed in this article are those of the authors and do not necessarily reflect the views of their employers.

* Corresponding author. Tel.: +32-2-771-00-14; fax: + 32-2-762-00-44.

E-mail address: jkleiner@ilsieurope.be (J. Kleiner).

$\dagger$ Chair of Individual Theme Group on Risk Characterisation. 


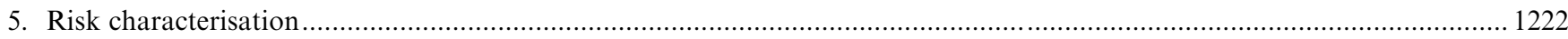

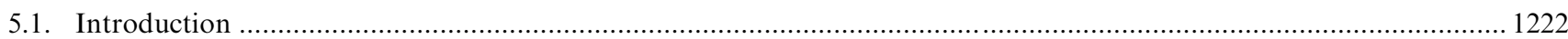

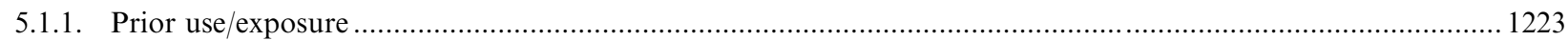

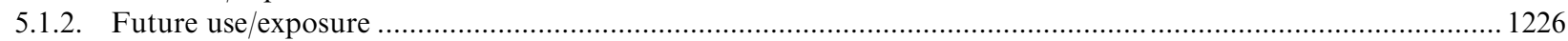

5.2. Integrating exposure assessment and hazard characterisation into risk characterisation ....................................... 1226

5.2.1. Matching the exposure assessment with the hazard characterisation .................................................... 1226

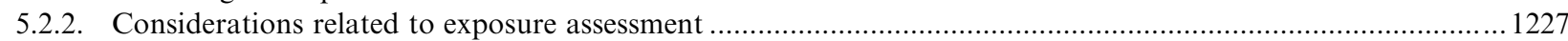

5.2.3. Matching the duration of intake measurement to the toxicity of concern ................................................. 12229

5.2.4. The use of biomarkers to define the relationship between external and internal dose, and to bridge the gap

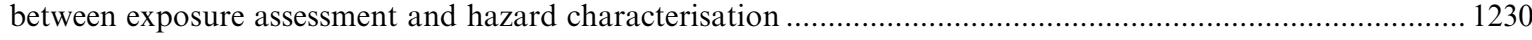

5.2.5. The influence of the nature of the hazard and hazard characterisation on risk characterisation....................... 1231

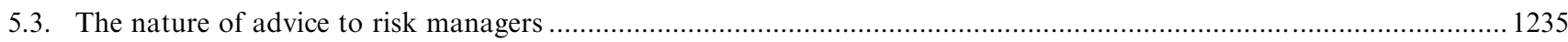

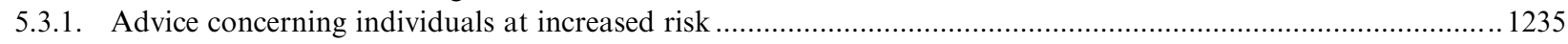

5.3.2. Advice related to simultaneous exposure to different chemicals in food .................................................. 1237

5.3.3. Advice related to different duration and patterns of human exposure......................................................... 1240

5.3.4. Advice when human exposure exceeds the health-based guidance value .................................................... 1242

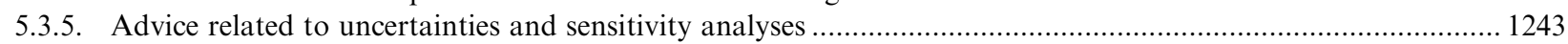

5.3.6. Advice relating to very low risks for irreversible hazards such as cancer............................................. 1247

5.3.7. Advice when data are sparse but indicate serious hazard ..................................................................... 1247

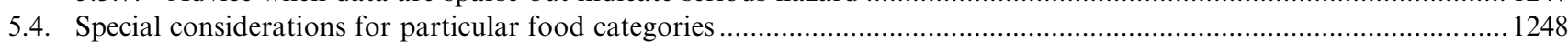

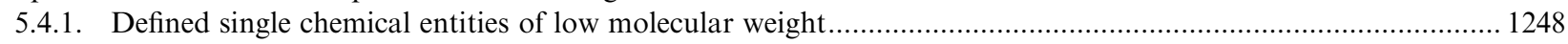

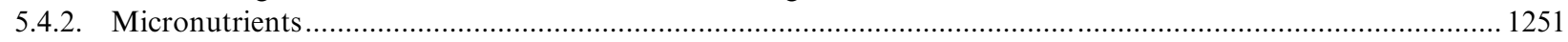

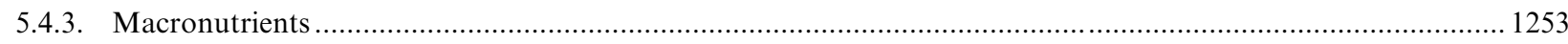

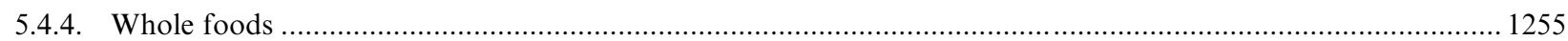

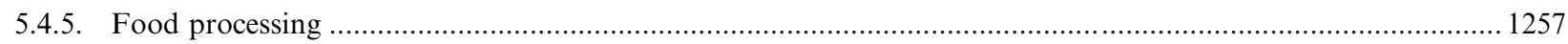

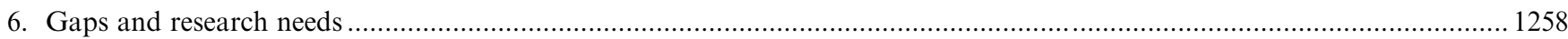

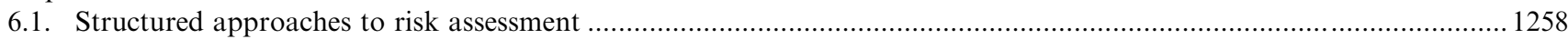

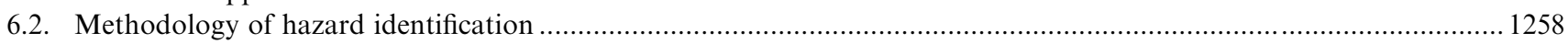

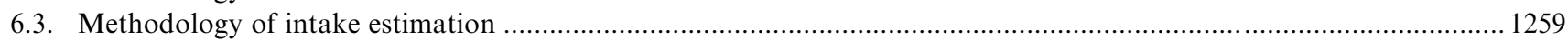

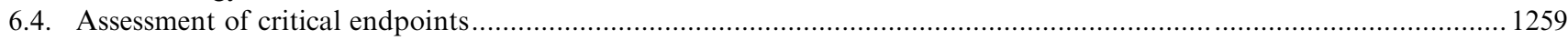

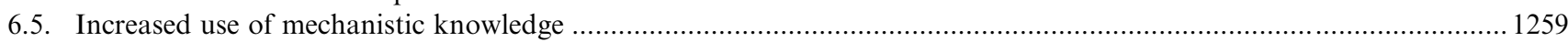

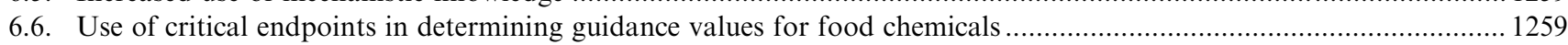

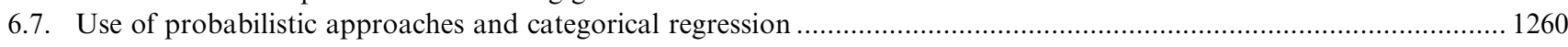

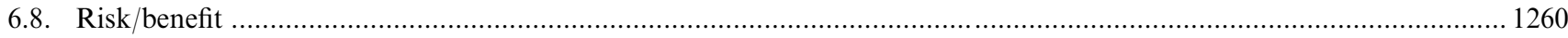

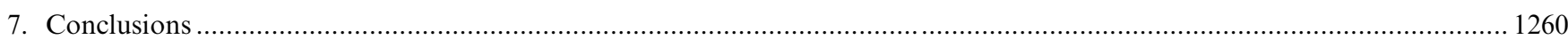

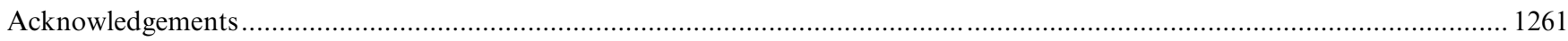

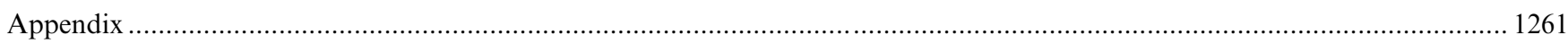

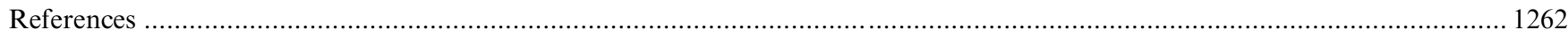

Abbreviations: 2,3,7,8-TCDD, 2,3,7,8-tetrachlorodibenzo-p-dioxin; ACNFP, Advisory Committee on Novel Foods and Processes (of the UK); ADI, Acceptable Daily Intake; ALARA, as low as reasonably achievable; ALARP, as low as reasonably practicable; ARfD, acute reference dose; AUC, area under the plasma concentration time curve; BMD, benchmark dose; COT, Committee on Toxicity of Chemicals in Food, Consumer Products and the Environment (of the UK); CSAF, chemical-specific adjustment factor; CYP1A2, CYP2D6, CYP2C19, cytochrome P-450 variants; DRI, dietary reference intake; EC, European Commission; EM, extensive metaboliser; EU, European Union; FAO, Food and Agriculture Organization of the United Nations; FDA, Food and Drug Administration (of the USA); FOSIE, Food Safety in Europe; GCP, Good Clinical Practice; GLP, Good Laboratory Practice; ILSI, International Life Sciences Institute; IPCS, International Programme on Chemical Safety; ITG, Individual Theme Groups; JECFA, Joint FAO/WHO Expert Committee on Food Additives; JMPR, Joint Meeting on Pesticide Residues (of the FAO and WHO); LOAEL, Lowest Observed Adverse Effect Level; LOQ, limit of quantification; MOE, margin of exposure; MOS, margin of safety; MRL, maximum residue level; NAEL, No Adverse Effect Level; NOAEL, No Observed Adverse Effect Level; OECD, Organisation for Economic Cooperation and Development; PCB, polychlorinated biphenyl; PCDD, polychlorinated dibenzo-p-dioxin; PCDF, polychlorinated dibenzofurans; PM, poor metaboliser; PRI, population reference intake; RfD, Reference Dose; SCF, Scientific Committee on Food (of the EC); STMR, supervised trials median residue level; TD50, the dose giving a 50\% tumour incidence in an experimental study; TDI, Tolerable Daily Intake; TEF, toxic equivalency factor; UK, United Kingdom; UL, upper intake level; USA, United States of America; VSD, virtually safe dose; WHO, World Health Organization. 


\section{Executive summary}

This report presents a review of risk characterisation, the final step in risk assessment of exposures to food chemicals. The report is the second publication of the project "Food Safety in Europe: Risk Assessment of Chemicals in the Food and Diet (FOSIE)". The science underpinning the hazard identification, hazard characterisation and exposure assessment steps has been published in a previous report (Food Safety in Europe, 2002). Risk characterisation is the stage of risk assessment that integrates information from exposure assessment and hazard characterisation into advice suitable for use in decision-making. The focus of this review is primarily on risk characterisation of low molecular weight chemicals, but consideration is also given to micronutrients and nutritional supplements, macronutrients and whole foods. Problem formulation, as discussed here, is a preliminary step in risk assessment that considers whether an assessment is needed, who should be involved in the process and the further risk management, and how the information will provide the necessary support for risk management. In this step an evaluation is made of whether data are available and what level of resources are needed, as well as the timeline for completing the assessment. The report describes good evaluation practice as an organisational process and the necessary condition under which risk assessment of chemicals should be planned, performed, scrutinised and reported. The outcome of risk characterisation may be quantitative estimates of risks, if any, associated with different levels of exposure, or advice on particular levels of exposure that would be without appreciable risk to health, e.g. a guidance value such as an acceptable daily intake (ADI). It should be recognised that risk characterisation often is an iterative and evolving process.

Historically, different approaches have been adopted for the risk characterisation of threshold and nonthreshold effects. The hazard characterisation for threshold effects involves the derivation of a level of exposure at or below which there would be no appreciable risk to health if the chemical were to be consumed daily throughout life. A guidance value such as the ADI, is derived from the no-observed-adverse-effectlevel (NOAEL) or other starting point, such as the benchmark dose (BMD), by the use of an uncertainty or adjustment factor. In contrast, for non-threshold effects a quantitative hazard estimate can be calculated by extrapolation, usually in a linear fashion, from an observed incidence within the experimental doseresponse range to a given low incidence at a low dose. This traditional approach is based on the assumption that there may not be a threshold dose for effects involving genotoxicity. Alternatively, for compounds that are genotoxic, advice may be given that the exposure should be reduced to as low as reasonably achievable (ALARA) or practicable (ALARP). When a NOAEL can be derived from a study in humans, this would be utilised in the derivation of guidance values or advice. However, there may be uncertainties related to the possible role of confounders and the precision of both the incidence and exposure data. Individuals may be at an increased risk because of their greater exposure or their greater sensitivity. Risk characterisation should include information not only on the general population, but also on any subpopulation considered to be potentially susceptible.

Risk characterisation considers both individuals with average exposures and those with high exposures. High exposure may be related to life stage, cultural practices and/or qualitative and/or quantitative food preferences. Inter-individual differences in toxicokinetics are an important source of variability in response. This may arise from differences in genetic constitution or environmental influences including diet, nutritional status, physiological status such as pregnancy, as well as pathophysiological states. Studies undertaken for hazard identification and characterisation investigate a substance in isolation, and not in combination with other substances to which humans may be exposed at the same time. It is recognised that food represents an extremely complex mixture of substances. In general, the available data indicate that interactions between chemicals in food are unlikely to be a significant problem for health. However, attention needs to be focused during risk characterisation on substances that share a common mode of action. The patterns of human exposure to chemicals in food may be chronic (usually lowlevel), short-term (often at higher levels) or chronic lowlevel with occasional high intakes. This may necessitate the development of guidance values for acute exposures (the acute reference dose, ARfD) based on shorter-term studies, in addition to an ADI-value usually based on chronic studies. The possibility of increased risks of chronic adverse effects associated with long-term lowlevel exposure, combined with occasional peak exposures has generally been handled by averaging such exposures. The significance of intakes above the ADI is difficult to assess. Consideration in this respect has to be given to the nature of the effect, the magnitude of the excessive intake, as well as the duration of excessive intake, in relation to the half-life of the compound in the body and the associated body burden. An intake above the ADI may not necessarily be associated with significant adverse health outcomes since the ADI usually is based on chronic intake and incorporates a safety margin. However, an intake above the ADI would have the effect of eroding the safety margin by the ratio of the ADI to the predicted excess intake. Alternative approaches to assessment of the significance of intakes above the guidance value are provided by 
categorical regression analysis and probabilistic methods. For non-threshold effects, such as for some cancers, that have undergone risk characterisation by the use of quantitative low-dose hazard extrapolation, any increase in risk with increased exposure can be readily interpreted using the same mathematical model.

The narrative that accompanies the risk characterisation should explain the strengths and limitations of the data. When risk characterisation is based on animal data, the validity of such data needs to be affirmed and reported. Also, uncertainties associated with the extrapolation of data from studies in animals to predict human risk should be presented. Uncertainty can be expressed numerically when intake assessment and hazard characterisation are based on mathematical calculations and/or empirical distributions. Such numerical analyses can also be subject to sensitivity analyses, to test the contribution of different aspects of the database to overall uncertainty. Knowledge regarding the influence of human genetic polymorphisms on toxic responses is advancing rapidly. This has led to increasing concern that the default uncertainty factor may not provide adequate protection in the case of certain polymorphisms. The methods used for risk characterisation of low molecular weight chemicals are applicable in many respects to micronutrients. However, there are some unique aspects, the most obvious ones being that some intake is essential for life and the margins between essential intakes and toxic intakes may, for a number of micronutrients, be small. Since both deficiency and excess of a micronutrient can cause health problems, two guidance values for a micronutrient may be expressed. The setting of a tolerable upper intake level (UL) includes consideration of what does not cause physiological perturbations as well as consideration of the probability of an adverse effect occurring at some specified level of exposure. Macronutrients, such as dietary lipids, proteins and carbohydrates, may be present in the food/diet in substantial amounts. Consideration needs to be given in hazard characterisation of macronutrients to tolerance and to toxicological and nutritional impact. Hazard characterisation using animal studies may not be possible because the addition of bulky macroingredients to experimental diets, in amounts that are exaggerated relative to the human diet, may render such diets unpalatable and/or cause nutritional imbalance. Because of this, the role of human trials and observational studies are widely viewed as particularly important for macronutrients, addressing toxicokinetics, nutritional issues and tolerance. Observational epidemiological studies may also help to identify adverse effects. As for micronutrients, there may need to be more than one guidance value for a macronutrient. In certain instances, a margin of safety approach may be preferable, when it is not possible in animal studies to exaggerate the dosage sufficiently to accommodate the usual uncertainty factors. This project also addresses hazard and risk characterisation related to whole foods, except those based on GM technology, which is dealt with in another European Union (EU) project, ENTRANSFOOD. Whole foods may be foods currently on the market or novel foods for which approval is being sought. There is as yet no world-wide consensus on the most appropriate approaches to hazard and risk characterisation of whole foods, other than to recommend that a case-by-case consideration and evaluation is needed. The initial approach to novel foods requires consideration of the extent to which the novel food differs from any traditional counterparts, or other related products, and hence whether it can be considered as safe as traditional counterparts/related products (the principle of substantial equivalence). As for macronutrients, epidemiological data identifying adverse effects, including allergic reactions, may also exist. Human trials on whole foods, including novel foods, will only be performed when no serious adverse effects are expected. The report ends with a discussion of knowledge gaps in the area of risk characterisation of exposures to chemicals in food and diet, and presents a prioritised list of research needs.

\section{Introduction}

In response to the need for clarification and improvement of the risk assessment process, the International Life Sciences Institute-European branch (ILSI Europe) elaborated a project proposal for a European Concerted Action to examine critically and to develop further the methodology of risk assessment.

The analysis of risk from the consumption of food is a complex and emotive issue that attracts much attention. The process of risk analysis is divided into three main elements (risk assessment, risk management and risk communication). In this document the focus is on risk assessment as a scientific evaluation process. Consideration of the processes of risk management, risk communication and risk perception did not form part of this project. It goes without saying, however, that it is of great importance that all stakeholders involved in the risk analysis process communicate extensively concerning the different elements. In this document, communication is identified as being of particular relevance in 'problem formulation', a preliminary step in risk assessment that considers and defines the questions, issues and the plan of the process for analysing and characterising the risk. In addition, following completion of the risk assessment, the risk assessors have the responsibility to communicate the estimated risks and their meaning clearly, including the assumptions made and the uncertainties underlying the numerical determinations. 
This document reviews the risk characterisation of chemicals in food. The science underpinning the first three steps of the risk assessment process (hazard identification, hazard characterisation and exposure assessment) has been published in a previous report (Food Safety in Europe, 2002).

\subsection{Aims of the project}

These have been outlined in detail previously (Smith, 2002) and will be reviewed only briefly here.

The project "Food Safety in Europe: Risk Assessment of Chemicals in the Food and Diet" (Acronym: FOSIE) is an EU Fifth Framework Programme Concerted Action funded by the European Commission, Quality of Life and Management of Living Resources Programme, Key Action 1 on Food, Nutrition and Health.

The aims of the project can be briefly summarised as:

- to explore means of improving the principles applied to, and scientific basis of, risk assessment with respect to food additives and contaminants, micronutrients and nutritional supplements, macronutrients and whole foods,

- to consider possible interactions between individual chemicals and effects of the food matrix,

- to identify the gaps in knowledge that might lead to differences in interpretation of toxicological and exposure data, and the research needs to reduce these,

- to determine the nature and level of testing needed for safety evaluation relevant to the nature of the chemical, level of use/occurrence in the diet and human exposure,

- to add a European contribution to international initiatives to harmonise principles, terminology and methodology for risk assessment,

- to contribute towards a consensus on risk assessment issues that is scientifically transparent and justifiable,

- to assist risk managers in developing appropriate, defensible food standards that adequately protect the safety of the consumer while allowing for innovation in food production and processing.

There is general agreement on the stages involved in risk assessment, though much of the work in this area originates from safety assessment of chemicals other than those found in foods. There is guidance for, and considerable experience with, the risk assessment of low molecular weight chemicals in foods, such as food additives and pesticides (World Health Organisation, 1987, 1990, 1994) and procedures are continually reviewed and updated. However, there is less experience with some other categories of food chemicals (i.e. micronutrients and nutritional supplements, macro- nutrients and whole foods). Guidance is still needed on how the risk assessment should be performed in practice, on the requirements of each stage of the process, and on the overall integration of the data obtained in each step.

\subsection{Way of working}

In the FOSIE project, individual theme groups (ITGs) were formed to assess the current state of the art, to establish the science base for new risk assessment methodologies and to identify gaps in current knowledge and the research needs to fill these gaps. The ITGs were designed to address all the steps involved in risk assessment. However, it was recognised that such a restrictive separation should not limit the coverage of the essential science, and the content of the ITG reports is, in some cases, much broader than inferred by their titles. Six ITGs were organised to consider the first three steps of the risk assessment process as follows:

- hazard identification by methods of animal-based toxicology,

- methods of in vitro toxicology,

- hazard characterisation of chemicals in food and diet: dose-response, mechanisms and extrapolation issues,

- mathematical modelling and quantitative methods,

- assessment of intake from the diet,

- the contribution of epidemiology.

The reports from these six groups were published in a separate issue of Food and Chemical Toxicology (Food Safety in Europe, 2002).

A final ITG was convened to consider risk characterisation and was composed of recognised experts in the field. They began their work by reviewing the information from previous ITGs. Individual sections were then prepared on the essential elements of risk characterisation and group meetings were organised to review progress and revise the drafts. The risk characterisation sections were reviewed and amended at the third plenary meeting of the FOSIE project (with additional invitations made to risk assessment experts and some risk managers who had not participated in the project). The contents of the sections following this introduction provide an integrated, comprehensive and critical appraisal of the processes used for the characterisation and quantification of risk.

\subsection{Risk characterisation - the last step in the risk assessment process}

Risk characterisation has been defined as follows: (European Commission, Scientific Steering Committee, 2000): "The quantitative or semi-quantitative estimate, 
including attendant uncertainties, of the probability of occurrence and severity of adverse effect(s)/event(s) in a given population under defined conditions based on hazard identification, hazard characterisation and exposure assessment".

The risk assessment process is shown schematically in Fig. 1 as being a branched linear process culminating in the final integrative step of risk characterisation. In reality it is an iterative process where the analysis carried out has to fit the data that are currently available. On occasions there is the need to characterise risk in data-sparse situations and this will lead to the development of estimates of risk that are subject to considerable uncertainty; any uncertainties should be characterised and, where possible, quantified. If an estimate associated with high uncertainty is sufficient for risk management purposes then generation of additional data may not be necessary. However, if further refinement of the risk assessment is required then additional iterations of the process with additional data generation will be needed, with a concomitant decrease in the degree of uncertainty.

\subsection{Scope of the document}

This paper is targeted principally at risk assessors, although the information will also be of value to those involved in risk management and risk communication.

The document is organised to reflect the way that risk characterisation is carried out, beginning with the issue of problem formulation and working through the data needed to the final step of risk characterisation and the guidance values and/or advice for risk managers. Finally there is a review of future challenges with recommendations on gaps and research needs. It is the identification of the research needs and the subsequent co-ordination of work to address them that will lead to stimulation of methodological and other improvements in the process.

It is clear that there is a widely accepted and reasonably mature system for the risk characterisation of low molecular weight chemicals and this document inevitably focuses on these. However, consideration is also given to the applicability of the risk characterisation process to other categories. The four food categories considered were:

- chemicals of low molecular weight, or "low relative molecular mass", e.g. food additives, pesticides, contaminants,

- micronutrients and nutritional supplements, e.g. vitamins and minerals,

- macronutrients, e.g. proteins, lipids and carbohydrates,

- whole foods, e.g. new varieties of food plants or exotic fruits.

Novel foods have not been covered as a separate category, as this is a regulatory term relating to the degree of previous consumption of a food in a defined population.

Food processing is considered across all of the four food categories when relevant.

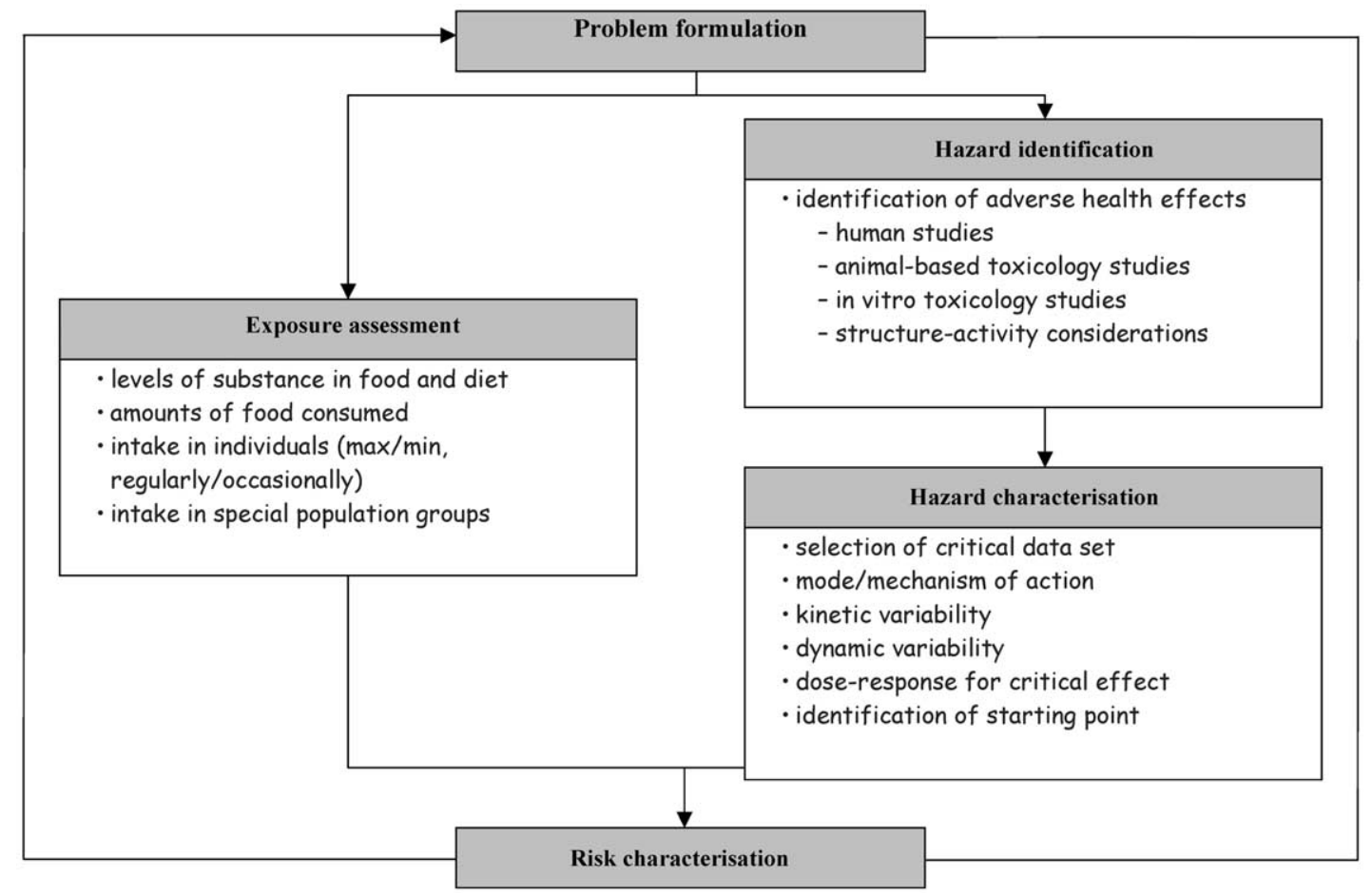

Fig. 1. The risk assessment paradigm. 
Others topics not covered in the document are:

- risk characterisation of the nutritional impact of macronutrients in the diet, for example in causing obesity. This is appropriate for dietary guidelines produced by many national and international authorities but outside the scope of this review;

- risk characterisation of genetically modified organisms and derived ingredients in food. A separate EU-funded thematic network called ENTRANSFOOD specifically covers this topic;

- risk characterisation of animal feed is only indirectly covered with consideration of components that may enter the human food chain;

- risk characterisation of microbiological risks for food;

- nutritional benefits of foods, these are also subject to a EU-funded project entitled PASSCLAIM (QLK1-2000-00086, http://europe. ilsi.org/passclaim);

- considerations of risk versus benefit.

\section{Problem formulation for risk assessment}

Problem formulation is the initial step in the whole risk assessment process. Although the focus of this paper is on risk characterisation, the final step of the risk assessment process, problem formulation is dealt with here in detail since risk characterisation needs to be in a form relevant to the problem identified initially. The outcome of problem formulation is an analysis plan with detailed questions for the risk assessor, on which the risk characterisation process has to focus. Ideally, problem formulation should be considered as an iterative process involving dialogue with all stakeholders, i.e. risk assessors, risk managers, manufacturers or producers, consumers, and it can develop as the risk assessment evolves.

\subsection{Process of problem formulation}

Problem formulation is the process by which the issues and questions are defined and the plan for analysing and characterising risk is developed. The process can be initiated by an individual outside the scientific and risk assessment community bringing a problem to public attention.

A clear formulation of the problem is critical for ensuring a useful and relevant end product of the risk assessment process. The first step to problem formulation is a planning dialogue that clarifies the management goals, the purpose and scope of the assessment and the resources available to conduct the assessment. The planning dialogue is an iterative process that considers:
- whether a risk assessment is needed,

- who should be involved in the risk assessment and risk management processes,

- how the assessment will provide the information necessary to support the risk management decision,

- whether data are available to embark on an evaluation of risks,

- what level of resources are available, and

- what is the timeline for completing the assessment.

Specific information to be gathered includes:

- a detailed inventory of prior knowledge,

- which individuals/population are the focus for the risk assessment,

- which (geographical) areas are to be covered, or which consumer setting,

- which route(s) of exposure are relevant, and

- which health endpoints should be considered.

Risk managers, risk assessors, and all stakeholders (from the initial producer/grower to the final consumer) bring valuable and often different perspectives to assessment planning. As an initial activity in problem formulation, information concerning potential exposure, known or suspected hazards, observed or hypothesised effects, potential risks, and modifying factors is collected. For existing food chemicals, e.g. those requiring re-assessment, prior knowledge (see Section 2.2 ) is of great importance. For new substances, the systematic collection of prior knowledge on any related substances may aid in the planning of a testing and research strategy.

At this stage, it may become clear that a detailed risk assessment is not necessary, for example when the exposures are at levels too low to be of concern (Kroes et al., 2000; International Life Sciences Institute, 2000a). The decision not to proceed with a full assessment will need to be communicated clearly to all interested parties.

If a risk assessment is deemed necessary, then feasibility must be addressed. Problem formulation should be as explicit as possible and should generally include considerations of relevance (including societal values), applicability (to the exposure scenario), and utility (to provide useful advice to risk managers). Existing data and the range of health endpoints can serve to identify quantifiable indicators or processes (measures of exposure, measures of effect, toxicokinetic or toxicodynamic data) to be used for the risk characterisation.

The process should undergo rigorous review by risk managers, scientific peers, and other stakeholders to ensure that all concerns have been addressed, and that the assessment will yield a scientifically sound and 
credible characterisation of risk. The methods to be used to evaluate the risk are ideally described in full in an analysis plan, which is the final product of problem formulation. Such analysis plans should indicate the information necessary for both risk assessors and risk managers to be satisfied that the assessment will provide the kind and quality of information necessary to make appropriate management decisions. In practice, such plans could require modification if new data emerge during the risk assessment process. The key point of the analysis plan is that it defines clearly the question(s) that the risk assessment has to address.

\subsection{Use of prior knowledge in problem formulation}

The collection and evaluation of key elements of prior knowledge, mainly regarding possible human exposure and possible hazards, are required for problem formulation and the subsequent decision (a) as to whether a risk assessment is necessary, and (b) whether further specific tests or studies could enhance the risk assessment. The initial step of the risk assessment process is the complete and systematic collection of all available information on a compound occurring in food. For each of the categories of chemicals in food and diet, as defined in Section 2.4, different types of information may be the most relevant.

A methodical approach to the collection of prior knowledge can be the first step in improvement of the current risk assessment process. In particular, the evaluation of all available information can bring into focus gaps in knowledge and be used to target further testing, population research, and exposure assessment studies or surveys, where needed. It can also assist planning to avoid duplication of effort and to reduce animal use. However, it is recognised that in some cases, where proprietary information is involved, access to prior knowledge may be precluded. Finally, the data compilation step can help in prioritising compounds and their reaction or degradation products for risk assessment. These considerations are frequently neglected; it is therefore recommended that systematic attention be given to them.

The following general topics are addressed in this context:

- prior knowledge on the substance,

- prior knowledge on exposure to the substance,

- prior knowledge on possible biological effect(s) of the substance.

It is important that, when collecting and evaluating data, as far as possible its reliability and relevance are assessed. For biological effects the strength of association should be taken into consideration and also its biological plausibility.

\subsubsection{Prior knowledge on the substance}

3.2.1.1. Origin of the substance: The first information should clarify whether the substance is of natural or synthetic origin. The substance can either be a defined chemical entity or a mixture of compounds, e.g. plant extracts.

If it is of natural origin, the source of the substance needs to be sufficiently characterised (for example, exact genus, species and subspecies for a plant bearing a toxin, anthropogenic or geological origins for a food contaminant such as arsenic, etc.). Agricultural practices should be considered as these may affect the levels of natural toxicants and contaminants, possibly also of nutrients. For micro-organisms used in the production of the substance a clear microbiological specification is necessary (including species, taxonomy, origin, etc.).

If the substance originates from a chemical or other form of production process, these have to be adequately described in order to obtain a clear specification of the substance and identify steps of the production process relevant to risk assessment.

Some substances may originate by formation during a food production process or during storage or preparation. In these circumstances it is important to collect available information on the physical or environmental conditions and the chemistry leading to the formation of the substance, as well as information on stages of the process at which its formation might be controlled.

3.2.1.2. History of use and consumption: In the case of a substance of natural origin, the history of human use (for example of a herbal product) or the extent of consumption or exposure via non-oral routes should be described. For substances of synthetic origin, the history of the synthesis or production process should be described, as well as its role in the production of foods or food components, to determine if the process per se raises novel concerns. Information on current and past use of the substance, including both food and non-food use(s), should be collected.

3.2.1.3. Chemical identity, characterisation and specification of the substance: The complete name of the substance, if possible according to official nomenclature, as well as all known alternative names or synonyms should be listed. Available analytical information should be evaluated to determine if it is sufficiently reliable to establish chemical identity, and/or to characterise the substance, including by-products and impurities. This is equally valid for synthetic and for natural compounds. For complex mixtures, such as plant extracts, a reliable and reproducible analytical fingerprint that sufficiently characterises the mixture should be available. The analytical information should relate to what is present in foods when the food is produced on a commercial scale, or when it is prepared in the home by consumers. On 
the basis of this information, appropriate specifications should be developed for identity as well as chemical and microbiological purity. All specifications need to be appropriate to ensure that the evaluated product is representative of that consumed, or alternatively, that any differences can be reliably taken into account.

\subsubsection{Effect of processing}

On the substance: qualitative and quantitative knowledge of the influence of food processing on the substance is needed to adequately address whether processing results in a significant change of chemical/ physicochemical identity and/or chemical and microbiological specification as compared to the non-processed counterpart.

On whole food: the presence of a novel substance in a food could modify the effects of a subsequent food production process on the quality and safety of the basic food constituents, for example a flour improving agent could deplete vitamins during dough processing. In some cases possible interactions between the substances, the food and the process may be deduced from knowledge of the chemical characteristics of the substance.

\subsubsection{Effect of transport and storage on the substance:} Information is necessary on the stability of the substance itself or as it occurs in food. In cases where defined storage conditions are necessary for the substance to remain stable, information is needed on these conditions and on the time periods over which the substance is stable and complies with its specification. The same is true for specific transport conditions if these are potentially influential.

\subsubsection{Prior knowledge on possible exposure}

The intended use or purpose of the substance or its occurrence as a contaminant or natural toxicant has to be defined clearly, additionally all existing qualitative and quantitative information on current and predicted exposures needs to be collected. For the oral exposure route, food and water are of relevance. Consideration of other possible routes of exposure, including non-food uses, and inclusion of these in the development of exposure estimates should be an essential component of problem formulation. As far as possible, data should include information about the amount consumed per person as well as information on the pattern of exposure for an individual (i.e. occasional, intermittent or regular consumption) and the pattern of exposure between individuals (i.e. variation across population groups). Another important consideration is whether predicted future exposure differs significantly from current exposure. The possible impact of a change in exposure has to be evaluated, for example consideration of all subgroups of the population when a substance becomes more widely consumed.
3.2.3. Prior knowledge on possible biological effect (s) of the substance

3.2.3.1. Qualitative aspects: All available information on possible adverse effects of the substance itself, its byproducts or impurities, or its contaminants should be considered. The available data should be assessed to determine whether one or several types of effects have been observed, and to identify those that are of health concern and of possible relevance to humans. Sources of such data are in vivo and in vitro toxicology studies, mechanistic studies, observational epidemiology studies (e.g. after occupational exposure) and randomised trials. The origin of the substance has to be considered, as well as the possibility that the source and/or the substance derived from it can contain compounds potentially detrimental to human health (including allergens). Data would be needed also on potentially adverse effects on human health from process-related changes in the substance or in the food containing it. Also of interest is any knowledge on interaction(s) of the substance with nutrients or toxicants in the diet, medicines, or other substances to which humans are exposed.

3.2.3.2. Quantitative aspects: For all of the above considerations, the availability of quantitative information needs to be examined. Where multiple effects have been observed, it may be useful to divide the health outcomes into those that are well-established and those for which data are suggestive but possibly more limited, more controversial or in need of greater scrutiny.

3.2.3.3. Predicted effects: If the substance is sufficiently described and characterised, but insufficient information is available on known effects, considerations can be given to the prediction of possible effects based on similar known and evaluated substances (e.g. structureactivity relationships). These comparisons can be done on a qualitative and a quantitative level, depending on available information and the degree of similarity to known substances.

Key features which should be sought in assembling data on prior knowledge are summarised in Fig. 2.

\begin{tabular}{|l|}
\hline 1. Prior knowledge on the substance: \\
origin of the substance \\
history of use and consumption \\
chemical identity, characterisation and specification \\
effect of processing on substance and on whole food \\
effect of transport and storage \\
2. Prior knowledge on exposure \\
3. Prior knowledge on possible biological effect(s) \\
qualitative aspects \\
quantitative aspects \\
predicted effects
\end{tabular}

Fig. 2. The systematic collection of prior knowledge. 


\subsection{Conclusion}

Problem formulation is the initial step in the risk assessment process and it is here that the stages of the assessment are defined and the plan for analysis and characterisation of the risk is developed. It is an iterative process requiring dialogue between stake holders. During problem formulation the collection and evaluation of prior knowledge is crucial in answering basic questions about human exposure and possible hazard and thereby allowing the determination as to whether a risk assessment is needed and whether it is possible. Detailed collection and evaluation of all available information may also help to prioritise and direct risk assessment, as well as to target research on exposure or health effects from chemicals in food. The output of problem formulation should be a detailed analysis plan in which the needs of the risk manager are made explicit at this early stage, as these are critical in defining the scope of work.

For either existing or new substances, the process of problem formulation can be described in the following scheme, Fig. 3.

\section{Good evaluation practice}

Good evaluation practice (GEP) defines the organisational process and the conditions under which risk assessments of chemicals should be planned, performed, scrutinised and reported. There are already guidelines as to how chemicals should be investigated in order to assess their potential to harm humans (Organisation for Economic Co-operation and Development, 2000). In addition, good laboratory practice (GLP) procedures (Commission of the European Communities, 1987, 1988) set out how such studies should be monitored and controlled for quality assurance purposes. GEP is intended to be complementary to these earlier processes and should increase the confidence of the public and the scientific community in the outcomes of risk assessment. Although not formally established, proposals for GEP have been published for consultation (Malmfors et al., 2001) and this section draws on the consultation document.

There are a number of steps of the evaluation process for chemicals that should be considered in the context of GEP. An important initial step is the procedure for forming the group that is to carry out the risk assessment. The majority of risk assessments are undertaken either for national or international organisations or within industry. However, these groups may have no formal existence beyond their meetings, or may have a need to bring in special expertise. It is therefore important that the procedures for the establishment of the group and for the co-option of additional expertise are defined. Information concerning the persons conducting the evaluation, such as their experience and affiliation, should be recorded. In particular, any possible conflicts of interest between members of the committee or staff of the organisation requesting the evaluation and the producer of the chemical/food in question must be recorded and made part of the public record. Similar records should be developed and retained within an industrial organisation for purposes of due diligence. Because risk characterisation requires extensive expertise in widely different areas it is likely that those involved will have gained their experience in relation to subjects, compounds or areas of work which may be under discussion. Therefore apparent conflicts of interest are probably inevitable in the establishment of groups undertaking risk characterisation and it is essential, for the purposes of ensuring the transparency of the process, that all those involved declare any possible conflict of interest.

A further stage in the evaluation is the technical process of identifying, collecting and categorising data and verifying their compliance with established guidelines or criteria. The importance of specifying the details of literature surveys, e.g. the databases scanned and search strategies employed, has been emphasised (Joint Expert Committee on Food Additives, 2000). Independent assessment of the quality of published papers retrieved during literature searches is important, notwithstanding the fact that most will have undergone peer review prior to acceptance for publication, because the primary aim of the study may not have been related to risk assessment. However, this assessment may be difficult, since journal publications generally contain fewer details than original study reports. Unpublished detailed study reports may be submitted to the group by industry; there is a need to recognise that although these may not have undergone independent external peer review, quality assurance is usually provided for modern studies by compliance with GLP. Such reports may have been submitted under conditions of commercial confidence; this must be respected but, if the data are from a healthrelated study, the organisation requesting the risk assessment should insist on the right to publish an abstract and analysis/review of the study. In such circumstances the study sponsor should be encouraged to place the study in the public domain but, in any event, the reviewing organisation should retain a copy of the report. Particular care is needed in the use of meeting abstracts, since the conclusions may change if the studies are published subsequently in full, peer-reviewed papers. Nevertheless, the conclusions from an abstract may have a role in the instigation of problem formulation by acting as a primary alert to a potential new hazard from food chemicals. It is also important to recognise the biases that may occur: firstly, towards publication of positive studies and non-publication of negative studies and, secondly, towards ignoring studies 
published in languages other than those familiar to the risk assessment group.

The quality of data from laboratory studies is also important. Considerations include whether studies have been conducted using internationally agreed protocols, according to GLP, with full reporting and appropriate statistical evaluation. Study reports should contain sufficient information to allow the reviewer preparing the risk characterisation to independently assess the validity of the results. It is also the responsibility of the reviewer to assess whether a study is scientifically appropriate to address the issue in hand. A well-conducted and wellreported study may be of little relevance to the problem formulation; nevertheless, an older study not performed to GLP may be scientifically sound and appropriate to the risk assessment. When reviewing human trials it is appropriate to consider assessing the compliance of the study protocol and the planning and conduct of the trial with the guidelines of Good Clinical Practice (GCP) as adopted by the United States Food and Drug Administration (Food and Drug Administration, 1997). A need of guidelines for Good Epidemiological Practice has
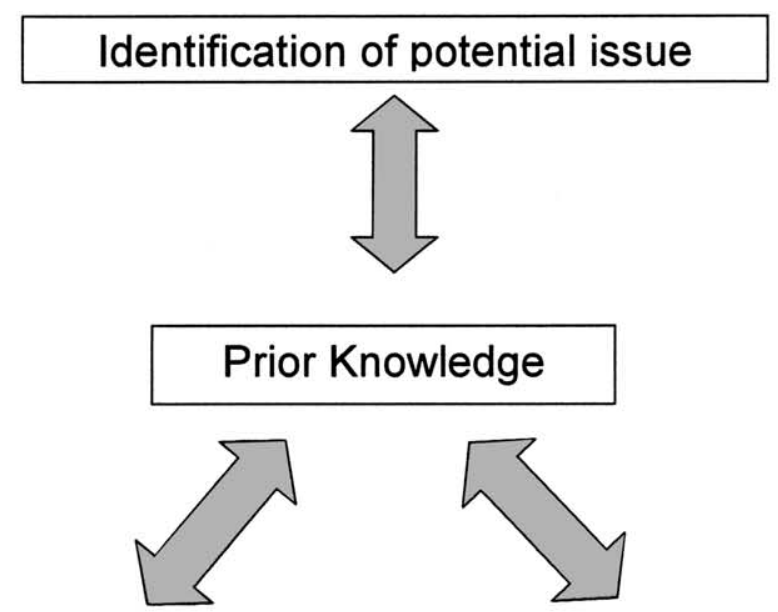

Prior Knowledge

Is there exposure?
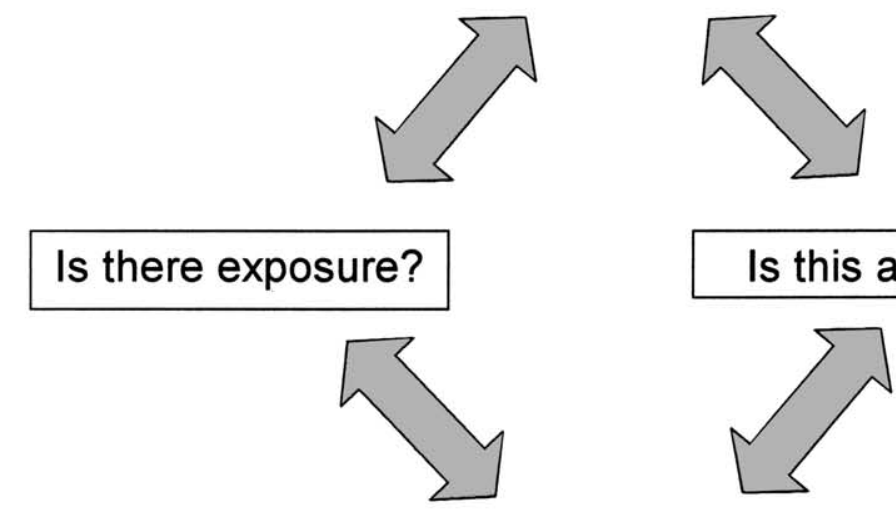

\section{Is Risk Assessment Necessary? \\ 2. Is Risk Assessment Feasible?}
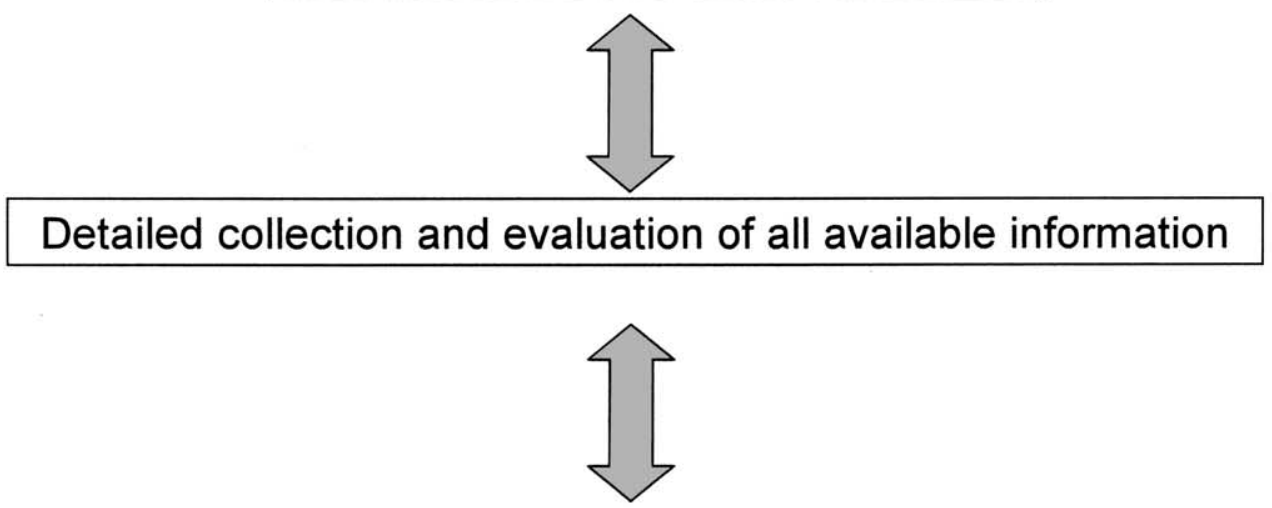

\section{Analysis plan}

Fig. 3. Problem formulation for risk assessment is an iterative process requiring a dialogue between stakeholders. 
been suggested (Baldwin and Hoover, 1991; Shore et al., 1992), as has their possible application to the publication of the results of epidemiology studies (Rushton, 2000). However, despite the publication of suggested guidelines (German Professional Society of Epidemiology, 2000), the matter has not progressed far.

In reviewing the available data it is important that its consistency should be considered. Thus, although a risk assessment might rely heavily on data from animal studies it may be that epidemiological data can be used to test the assumptions underlying extrapolation from animals to humans. Similarly, if the dose-response relationships from epidemiological studies are used for risk characterisation, in vitro research or in vivo studies in animals may provide a better understanding of the mode of action of the toxicant (Goldman, 2001; HertzPicciotto, 1995). Further, the biological plausibility of the selection of a critical effect from an animal study may be improved if there is evidence from other studies, either in another species or of shorter duration in the same species, which demonstrate either a similar effect or other changes which are consistent with the known biology of the critical effect.

The scrutiny and interpretation of data involve expert judgement. Since the exercise of expert judgement is a process in which scientific discipline and expertise, experience in risk assessment, affiliation, and national or international views are involved, it is not surprising that different evaluators sometimes reach different conclusions. To ensure that the judgmental part of the evaluation procedure is transparent, it is important not only that the evaluation is described in detail, but also that some indication is given of the process, including any factors which may influence its outcome. For those organisations conducting risk assessment on a regular basis, general procedures on how to perform the activities involved in the evaluation procedure should be described in writing in the form of standard evaluation procedures. It should also be the task of management to ensure compliance with the principles of GEP when evaluations are conducted and described. Preferably the organisation should use a documented quality assurance programme to ensure proper execution of the process.

Ideally, an evaluation should follow a systematic plan, in which the purpose of the evaluation is made clear to all involved, the time plan for the evaluation is set out and the evaluation methods to be used and the nature and extent of reporting of results are all agreed. The written outcome of the evaluation should include a description of the purpose of the evaluation, the methods used, the results, their interpretation and conclusions, together with a clear description of any uncertainties in the evaluation. The publication of clear written evaluations with detailed explanations for the decisions taken should minimise any criticisms of bias.
The risk assessment process is complex and the International Programme on Chemical Safety is in the process of developing recommendations about the harmonisation of procedures that are used. The ultimate aim of GEP is to improve the quality, reliability and transparency of evaluations in the risk assessment process. Although it is still in its infancy, GEP should mature in a systematic way in the coming decade and become as recognised and accepted as GLP and GCP.

\section{Risk characterisation}

\subsection{Introduction}

Risk characterisation is the stage of risk assessment that integrates information from exposure assessment and hazard characterisation into advice suitable for use in decision-making or risk management. The advice to risk managers may take the form of a quantitative estimate of the magnitude of any risk associated with different levels of exposure, such as the possible health consequences of adopting different regulatory limits in food (see for example aflatoxins; Joint Expert Committee on Food Additives, 1999). Alternatively, it can take the form of advice on particular levels of exposure that would be without significant adverse health effects (for example, setting a guidance value such as an ADI).

As data on exposure and hazard characterisation are collected and/or generated, new issues may be identified that were not recognised initially as a part of problem formulation. For example, new data revealing an increase in exposure may affect the need for data on hazard, and vice versa. In consequence, risk characterisation is often an iterative and evolving process, see Fig. 4.

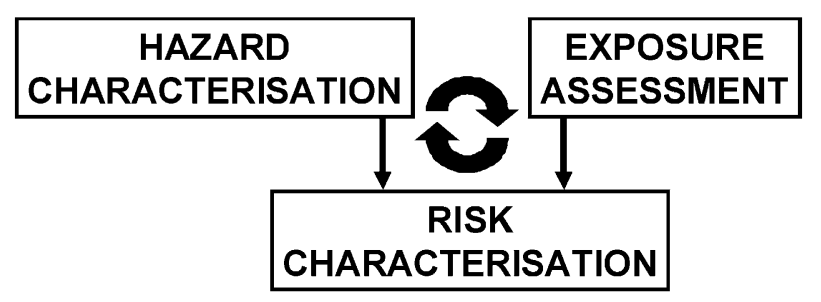

Is the exposure sufficient to warrant full hazard characterisation?

Is the hazard relevant to the exposed individuals?

Do the hazard characterisation data match the human exposure?

Integration of new data on hazard identification or characterisation

Integration of new data on increased or altered pattern of uses

Integration of new data on the nature of the distribution within food

Fig. 4. The iterative nature of risk characterisation, with examples of questions and information that can affect the outcome. 
An early step in developing the database for risk characterisation should be the inclusion of any prior knowledge on the chemical itself or related chemicals. This might include information on the nature of the chemical or substance, its specification, structural alerts for toxic actions, and knowledge of any structure-activity relationships with other, related chemicals. When prior knowledge, such as data on structural analogues, raises the possibility of a particular hazard, it is important to ensure that there are relevant and adequate data to address the issue (e.g. data on genotoxicity and carcinogenicity for chemicals with structural alerts for these endpoints, or immunotoxicity data for proteins, or human data in cases where problems with gastrointestinal tolerance may be anticipated). The use of prior knowledge has been discussed in more detail previously (see Section 3.2. above and Barlow et al., 2002).

Once all the available data have been gathered, consideration needs to be given to:

- whether exposure is sufficient to warrant a detailed risk characterisation and if so,

- whether there are sufficient data to make a thorough and meaningful appraisal.

The complexity of risk characterisation stems from the fact that conclusions may have to be reached in the absence of complete information. First, there is variability in the data that are used, both for exposure and effects, whether they derive from experimental or epidemiological studies. Secondly, there is uncertainty due to lack of complete knowledge concerning the parameters that govern the events observed in experimental and epidemiological studies, in particular, the fundamental underlying mechanisms of action. Identification and, where possible, quantification of variability and uncertainty are now regarded as important elements in risk characterisation. Because risk characterisation is an iterative process, see Fig. 4, the nature of the advice to risk managers may become more refined as the quality of the available exposure and hazard characterisation data increases. Risk characterisation is a complex process, with different approaches adopted for different types of hazard, as indicated in Fig. 5.

For adequate hazard characterisation the data should cover all the potentially relevant life-stages and toxicological endpoints, should enable the critical endpoint(s) to be identified, and should have sufficient dose-response information to establish dose levels with and without adverse effects. In an ideal situation, both laboratory and human data will be available, together with reliable information on exposure (although this will not be possible for new compounds/substances). Considerable attention is given to the selection of the adverse effect most appropriate and relevant to the nature of human exposure. One of the most important steps is the identification and characterisation of the critical effect(s), i.e. the hazard that is of greatest concern, both qualitatively and especially quantitatively, because this will be the main focus for the risk characterisation.

Theoretically, an ideal approach to risk characterisation might be to use in vitro data and in vivo studies in animals and humans for the identification of possible hazards, and then to use data from experimental or epidemiological studies in humans to characterise the hazard(s) and define the dose-response relationship(s). Because epidemiological research is done under realworld conditions in humans, the data can be of direct relevance to risk assessment and human health. In reality, for both practical and ethical reasons, observational epidemiological data are available in only a few cases, and therefore hazard characterisation usually is based primarily on data from in vitro studies and studies in animals. Animal toxicological data and epidemiological data are complementary sources of information, each of which has both strengths and weaknesses.

Risk characterisation may be separated into two different types, based on whether humans are exposed to the material at the time of risk characterisation (prior use/exposure), or whether exposure is not permitted until after completion of the risk characterisation process (future use/exposure). This subdivision is frequently reflected in the nature and extent of the data that are available for both exposure estimation and for hazard characterisation. Table 1 presents a summary of the main differences, using, as examples, a contaminant for prior use/ exposure and a food additive for future use/exposure.

\subsubsection{Prior use/exposure}

When there is human exposure at the time of risk characterisation, intake or exposure assessment can be based on actual measurements rather than predictions using anticipated or proposed patterns of use. The measurement of actual intakes has been discussed extensively in an earlier FOSIE publication (Kroes et al., 2002). The methods suffer from various uncertainties that should be described, where relevant, as part of risk characterisation, including:

- the extent to which all relevant sources of exposure can be taken into account,

- adequacy of any sampling strategy with respect to foods,

- sensitivity, accuracy and precision of any analytical techniques,

- adequacy of intake information with regard to representation of population subgroups defined by life-stage, ethnicity, nutritional status or health status,

- whether there is likely to be under- or overreporting in food consumption data, 
- scenario(s) selected for estimating high consumption,

- use of short-term data to predict longer-term exposure.

For existing food chemicals, it is important to gather all available data on hazard characterisation, including information obtained by searching the open literature. Appropriate and clearly described search strategies should be used that are designed to identify relevant experimental laboratory studies, human case reports, epidemiological studies and exposure data. Critical assessment of the quality of the data in published literature is important (see Section 4), because many studies would not have been performed to GLP standards. In addition, many published human studies would have been designed to investigate endpoints such as nutritional benefits rather than hazards, and therefore a critical appraisal of their suitability for the planned risk characterisation is essential.

The data available for hazard characterisation of some food chemicals, for example with natural toxicants, micronutrients, macronutrients and whole foods (see Section 5.4 respectively), may be limited animal data with

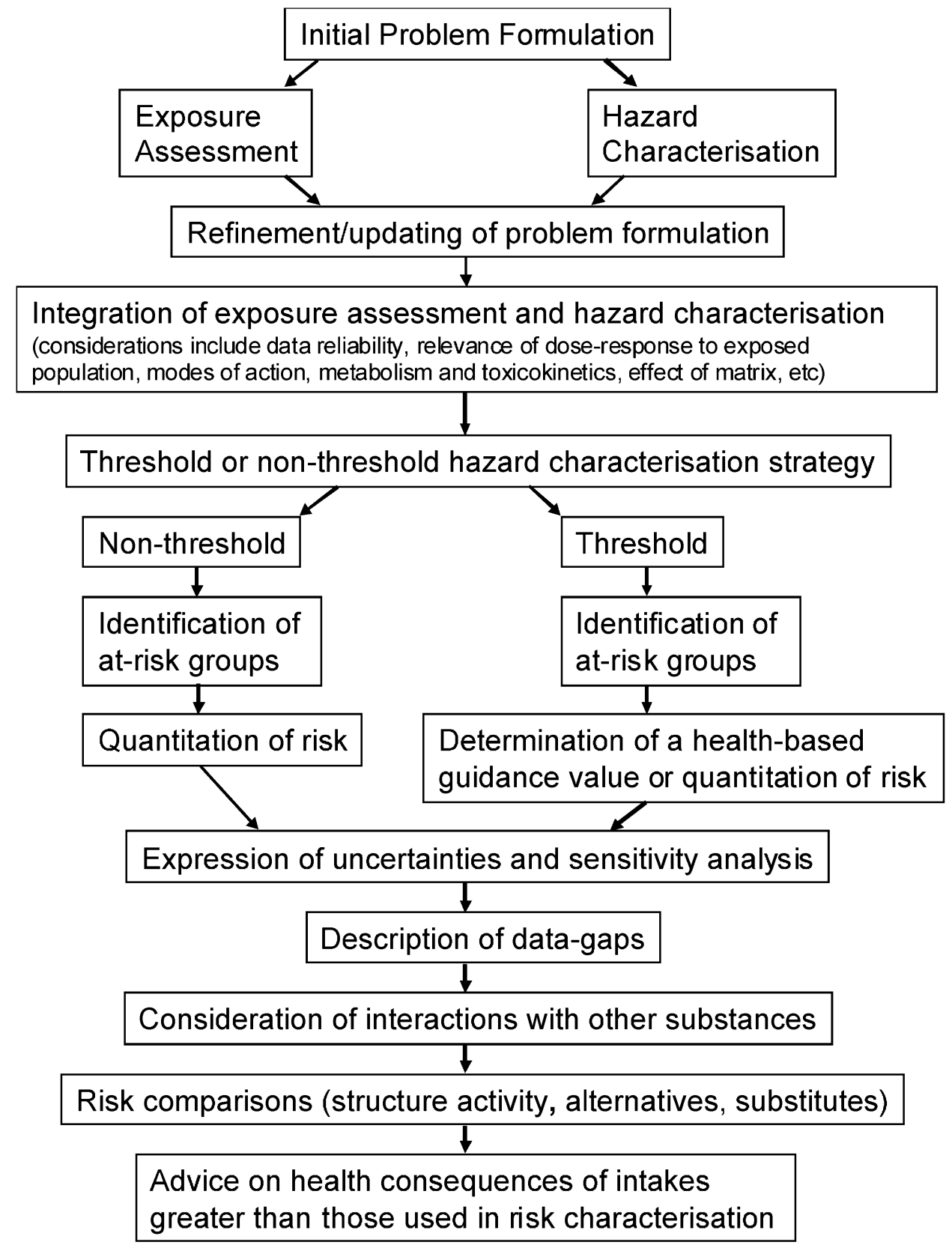

Fig. 5. Summary of the considerations that contribute to risk characterisation. 
some usable human data, including exposure data. For some inorganic contaminants, e.g. lead and cadmium, and organic contaminants, e.g. dioxins and polychlorinated biphenyls (PCBs), there are extensive laboratory and human epidemiological data, together with comprehensive exposure data.

In many cases risk characterisation may have to proceed in the absence of an adequate or complete data set. In such circumstances, and when the adverse effect is considered to show a threshold in the dose-response relationship (see below), additional uncertainty factors may be applied to the no observed adverse effect level/ lowest observed adverse effect level (NOAEL/LOAEL) to derive a health-based guidance value. Such factors are applied as part of hazard characterisation not risk management, because scientific expertise is needed to select the magnitude of the factor. Reviews of available toxicity databases have been used to derive the default uncertainty factors that may be applied to a LOAEL to allow for the absence of a NOAEL (usually 3 or 10) and to the NOAEL from a sub-chronic study to allow for the absence of a chronic NOAEL (usually 10) (Dourson and Stara, 1983; Dourson et al., 1996; Vermeire et al., 1999).

In contrast, the uncertainty factors that are used to allow for missing hazard identification data, for example the absence of reproductive or developmental toxicity data, or for the severity/adversity of an endpoint such as cancer (World Health Organization, 1994), are not scientifically based (Renwick, 1995). A more scientific approach to missing data would be to consider the probability that the NOAEL for the compound, based on other endpoints, would be adequate for the missing endpoint. This could be done by tabulating the NOAEL values for the missing endpoint for other compounds and determining the probability that the NOAEL for the missing endpoint for the compound under evaluation would be higher than the NOAEL that is based on other endpoints; an approach that is analogous to the threshold of toxicological concern (see Section 5.2.1.2). A number of databases, related to the development of thresholds of toxicological concern, have been developed for use in such analyses (Munro et al., 1996; Cheeseman et al., 1999; Kroes et al., 2000).

Table 1

Comparisons of the data available related to the extent of human exposure at the time of risk characterisation

\section{Prior use/exposure (e.g. for a contaminant)}

Exposure assessment

- Distribution within the food supply needs to be determined

- Concentrations in different foods need to be measured

- Concentrations in foods may show large variations

- Any unit-to-unit variability in the concentration present has to be allowed for when considering peak exposures and short-term effects

- Because the presence in food is not "permitted" and subject to prior approval, the total intakes may exceed the health-based guidance value; advice to risk managers may need to indicate the magnitude of any risk as well as the need for possible remedial measures

\section{Hazard characterisation}

- Many studies in animals are not performed to internationally accepted protocols or to GLP (good laboratory practice), and may have limited use for regulatory purposes

- Epidemiology data may be available for hazard identification and/or hazard characterisation

- Dose-response assessment may be based on the data from observational epidemiology studies or toxicity studies in experimental animals

- Uncertainty factors may be necessary if the population studied epidemiologically does not include all subgroups

- Additional uncertainty factors are often necessary to allow for deficiencies in the database
Future use/exposure (e.g. for a food additive or pesticide)

\section{Exposure assessment}

- Distribution within the food supply is defined by proposed uses

- Concentrations in different foods are based on technological need and are specified in the application for approval

- The maximum concentrations (or residue levels for a pesticide) in different foods are known prior to approval

- Intake estimates use the planned proposed maximum permitted concentrations or residue levels combined with food intake data to estimate a realistic worst-case intake; the planned maximum concentrations and/or food uses would not (all) be permitted if they resulted in an intake above the health-based guidance value

- Because the presence in food is "permitted" subject to prior approval, the total intakes should not exceed the health-based guidance value; if post-launch monitoring indicates excessive total intakes above the guidance value, the profile of approval could be revised.

\section{Hazard characterisation}

- Hazard identification and/or hazard characterisation depend on in vitro studies and in vivo investigations in test animals performed to internationally accepted protocols and GLP

- Human trial data, from experimental studies can assess tolerability but not safety; metabolism-toxicokinetic data can facilitate inter-species extrapolation

- Dose-response assessment is usually based on toxicology studies in experimental animals

- Uncertainty factors related to species differences and human variability are normally required

- Additional uncertainty factors to allow for deficiencies in the database are not normally used, as the applicant will be requested to supply any missing data prior to approval 
Because the individual uncertainty factors refer to unrelated events, they are multiplied. The composite uncertainty factor assumes that each area of uncertainty is independent; the use of large uncertainty factors may result in risk characterisation that could be highly conservative. The application of very high overall uncertainty factors $(>10,000)$ to databases that have multiple deficiencies is considered by many to render a risk characterisation meaningless (World Health Organization, 1994).

The most reliable examples of risk characterisation associated with prior exposure are probably when there is re-appraisal of an approved compound, such as an additive or pesticide, for which there is an extensive history of use, combined with a comprehensive hazard characterisation database.

\subsubsection{Future use/exposure}

Usually in the case of chemicals intentionally developed for agricultural or food uses (additives, flavourings, processing aids, pesticides, food contact materials), hazard characterisation must rely on comprehensive laboratory data from in vitro studies and in vivo studies in experimental animals because only limited human data are likely to be available. Intake estimates have to be based on predictions related to the proposed or anticipated uses, rather than intakes measured under real life conditions.

There are well-established procedures for situations in which problem formulation and risk characterisation precede human exposure. In the case of new food chemicals that require authorisation prior to marketing, the company wishing to market the chemical is usually required to supply most of the information for risk assessment. Dossiers submitted to regulatory authorities will normally contain extensive data on hazard identification and characterisation that can be used for risk characterisation. Intake and exposure estimates have to be based on the expected concentrations in different foods and the anticipated intakes of the foods for which approval is sought.

Regulatory authorities generally have powers to request submission of missing or additional data to clarify any issues that are not resolved by the available information. When pre-marketing approval is required, as for additives or pesticides, there is an increasing tendency for advisory bodies to withhold approval of a new substance until all the necessary data are available, rather than give temporary approval with the utilisation of additional uncertainty factors. In some cases, a temporary ADI or a temporary tolerable daily intake (TDI) may be allocated when there are uncertainties associated with the hazard characterisation, which are minor in extent and would not affect risk characterisation over the relatively short time period required to generate and evaluate appropriate data. Under such circumstances an extra uncertainty factor of 2 is usually applied to give a larger overall uncertainty factor for deriving the guidance value (see threshold approaches below).

\subsection{Integrating exposure assessment and hazard characterisation into risk characterisation}

Integration of the exposure assessment (based on the exposure scenarios that were the basis for problem formulation) with hazard characterisation needs to consider a number of key aspects, the information for which may evolve as the data are generated and/or collected.

\subsubsection{Matching the exposure assessment with the hazard characterisation}

5.2.1.1. How much hazard information is needed? The extent of hazard identification and hazard characterisation data needed for risk characterisation depends on the nature of the issues raised in problem formulation, and the anticipated human exposure. For example, it may not be necessary to have a comprehensive database in cases where there is a low potential hazard, as predicted by structure-activity relationships with structural analogues, and exposure is anticipated to be very low. This consideration forms the basis of tiered approaches to toxicity testing, in which the amount of hazard identification and hazard characterisation data required by regulatory bodies may increase as anticipated exposure increases. This pragmatic approach recognises the conflict between the desirability of having a comprehensive hazard characterisation database and the desirability of minimising unnecessary studies in animals and humans, as well as avoiding the use of scarce research resources for exposures of low priority: or, to put it more simply, risk characterisation processes attempt to recognise the difference between "need to know" and "nice to know".

\subsubsection{Threshold of toxicological concern: Biological} considerations and empirical evidence are consistent with the majority of chemically-induced adverse effects having levels of intake or exposure below which no lasting disturbances of physiological or biochemical function are observed. This has led to the development of the concept of a "threshold of toxicological concern", which represents a level of human exposure below which it can be considered there are no significant risks to health, even in the absence of data on the compound under evaluation (Kroes et al., 2000; ILSI, 2000a). Where such an approach is adopted there is no need for information on hazard identification and hazard characterisation, providing that predicted or actual exposures are below the threshold value. In the dietary field, these principles have been applied to the safety evaluation of flavouring substances and food packaging substances. For instance, the safety 
evaluation of flavouring substances by the Joint FAO/ WHO expert committee on food additives (JECFA) is based on a decision tree that takes into account chemical structure (Cramer et al., 1978), predicted metabolism and estimated exposure (Joint Expert Committee on Food Additives, 1995; Munro et al., 1996). A flavouring substance on which there are no actual toxicity data can be considered to be of no safety concern, providing that the intake is below the appropriate threshold of toxicological concern that is incorporated into the decision tree. The adoption of such an approach for this category of food chemicals is only considered legitimate because of the generally very low exposures to flavouring substances.

Additional consideration needs to be given when structure-activity considerations suggest the possibility of an effect such as genotoxicity, for which there may not be a threshold in the dose-response relationship (see below, and Edler et al., 2002). This possibility has been taken into account in the risk characterisation of another group of food chemicals with potentially low exposures, food packaging migrants. A "threshold of regulation" is used by the US food and drug administration (FDA) to review components of food contact materials with low exposures. The threshold is set at a dietary concentration giving a daily intake of $1.5 \mu \mathrm{g}$ per person. Below this threshold the FDA requires no specific toxicity testing and performs only an abbreviated safety assessment (Food and Drug Administration, 1995). The FDA threshold of regulation was supported by an analysis of the potencies of chemicals that had been studied in chronic cancer bioassays in rodents. A highly conservative, linear low-dose extrapolation from the $\mathrm{TD}_{50}$ (the dose giving a $50 \%$ tumour incidence in the experimental study) was used to derive the dietary concentration predicted to be associated with a risk of cancer of one in a million or less (Food and Drug Administration, 1993). The concentration which gives a human daily intake of $1.5 \mu \mathrm{g}$ per person was estimated to be $0.5 \mu \mathrm{g}$ per $\mathrm{kg}$ of diet. Above the threshold of regulation, a tiered approach to toxicity testing of food packaging substances is used by the FDA, and a similar tiered approach is used by the EC Scientific Committee on Food (Scientific Committee on Food, 2000a).

An extension of the threshold of toxicological concern concept took into account the probability of an unstudied chemical being a carcinogen (Munro, 1990), and was used to derive a threshold of $1.5 \mu \mathrm{g}$ per person per day. This threshold was incorporated into the JECFA decision tree for the safety evaluation of flavouring substances, and applied, for example, to the safety evaluation of allyl 2-furoate (Joint Expert Committee on Food Additives, 1999). Further possible refinements to allow application of the approach to a wider range of food chemicals are currently under discussion in an ILSI Europe Expert Group.
Chemical-specific data on both exposure and hazard are necessary when the actual, or predicted, human intake exceeds the threshold of toxicological concern, or is deemed to be significant for some other reason, such as structure-activity considerations.

\subsubsection{Considerations related to exposure assessment}

Intake issues important in risk characterisation are summarised below; a comprehensive account is given in an earlier FOSIE publication (Kroes et al., 2002).

\subsubsection{Intake estimates in relation to intake patterns:} The reliability of risk characterisation is dependent on the quality of the exposure data relevant to the issues that were identified in problem formulation. As exposure/intake data are collected they may reveal additional issues, such as the existence of highly exposed subgroups of the population, which need to be taken into account in risk characterisation.

Initial estimates of human exposure may need to be refined following hazard identification and characterisation. A number of important questions relating to intake patterns should be considered, e.g.:

- Are foods containing the substance consumed regularly or only occasionally?

- Does intermittent exposure justify the calculation of mean intakes over time, or should risk characterisation be based on intakes during the periods of actual consumption?

- Is intake relatively constant, allowing averaging of intake levels, or is there regular intake combined with occasional acute peaks, which may have health relevance?

- Is high consumption limited to specific lifestages, e.g. infant foods, or are relevant products consumed in the context of targeted diets, for example the intake of products containing phytosterols to lower blood cholesterol?

There are two types of information necessary for the generation of intake data suitable for risk characterisation. Firstly, determination of the consumption of the foods that may contain the chemical and, secondly, the concentrations of the chemical that may be present in the different foods. The intake estimate for a chemical or ingredient arising from ingestion of any single food item is the product of the concentration of the chemical/ ingredient in the food and the amount of the food consumed. The total intake for an individual from food is the sum of intakes from all relevant foods.

An additional consideration is whether all relevant sources of exposure have been identified, i.e. not only the foods expected to contain the component in question, but also any non-food sources of exposure. These include drinking water, consumer products, occupational sources 
and general environmental sources. For some chemicals, assembling all of the data needed to estimate total exposure may be difficult.

\subsubsection{Measurement of the intakes of foods that may contain the chemical}

Prior use/exposure: There is a hierarchy of methods for estimating intake, which offers increasing reliability and relevance, but at the cost of increasing complexity and expense. Food consumption and chemical intakes can be estimated at the supranational level (e.g. the intake of flavours within Europe based on annual production statistics), at the national level (e.g. national per capita estimates), for a household (e.g. market basket surveys) or for the individual (e.g. a food diary).

Food consumption estimated at a national level is based on food balance sheets integrating production, import/export, changes in stocks, and use at all levels in agriculture, industry and trade. This approach can give only an approximation of the intake, but this may be sufficient for risk characterisation when the potential exposures and possible risks are very low.

Household survey data are more reliable and more relevant. They are based on the total purchases of foods by a household, taking into account unused amounts (e.g. processing losses or spoilage). They do not provide an estimate of consumption by individual household members. So-called "market basket" studies are surveys that model the purchase and consumption habits of the population in general and/or subgroups defined by age or sex. They can be based on generic foods or alternatively on the most commonly consumed items in individual food categories.

The most reliable and useful data for risk characterisation come from dietary surveys in individuals. The simplest method is a recall study, in which the types and amounts of foods consumed during the last one, or several days, or the frequency of foods consumed in the past are recorded. Clearly, such data become less precise the longer the period of retrospective assessment. Prospective diary studies documenting the actual consumption during the observation period are more precise. A combination of dietary recall plus a food diary may be the most pragmatic approach (Lambe et al., 2000).

A particularly reliable but intensive method for assessing the intake of contaminants is by collection of a duplicate diet, where the same amounts and types of food consumed are also collected and subsequently analysed for the contaminant. Such studies can integrate both consumption and compositional data for the particular individual and life-stage or lifestyle.

A common problem for risk characterisation is that food intake surveys may not cover the full range of potentially exposed population subgroups. For example, infants or the elderly may not be represented.
Similarly, ethnic minorities whose dietary habits vary substantially from the main ethnic group may be inadequately sampled in the studies on which the intake information is based. Finally, intake by persons with medical conditions, some of which may affect food consumption may not be well characterised in typical food surveys. Even for well-sampled populations, consumption measurements suffer from inaccurate reporting: there is often under-reporting based on total food energy measurements, with a reporting bias in favour of "healthy" foods, such as fruits and vegetables, and against "unhealthy" products like alcoholic beverages or sweets. These uncertainties need to be described in the advice given to risk managers.

Future use/exposure. For substances that undergo risk characterisation before they are permitted to be used, such as food additives, veterinary medicines and pesticides, the potential intake has to be estimated based on the proposed uses. This has to take into account the different food types that could contain the substance and the concentrations that would arise from the technological use of the substance in the food or, for a pesticide or veterinary medicine, the concentration that could be present as a residue in food.

For food additives, simple and highly-conservative, "budget" methods can be used as an initial screen to determine if more sophisticated analyses are necessary (Kroes et al., 2002). For example, the maximum theoretically possible intake can be calculated if it is assumed that the additive will be present in all foods for which use is requested at the maximum intended concentration and using the maximum likely consumptions of relevant food categories. The results of this 'worst case' method determine whether more reliable estimations are necessary. If the intakes calculated by this method are below the ADI, undertaking more complex, but realistic calculations would be unnecessary. More realistic intake estimates are necessary for additives where the intakes predicted by simple conservative methods are close to or exceed the ADI, or where there may be subgroups within the population with high predicted intakes. Such calculations should aim to predict the intake by individuals with both average and high consumption of food chemicals.

Risk characterisation of agricultural pesticides is undertaken in a manner analogous to that of food additives. The maximum residue level (MRL) permitted on crops is based on the results of field trials conducted under the proposed conditions of maximum agricultural use; food containing residues at or below the respective MRLs are considered to be toxicologically acceptable for chronic intake (World Health Organization, 1997). More recently, however, it has been recognised that the use of the MRL in intake calculations is overly conservative for chronic risk assessment, and now the supervised trials median residue levels (STMR) are used. 
The STMR is the median residue level measured in a food crop treated with a pesticide according to good agricultural practice, at the maximum permitted use levels. The predicted daily intakes are compared with the ADI, and the estimated short-term intakes are compared with the ARfD (Section 5.3.3.1). As for food additives the requested uses of the compound would not be approved if the predicted intakes by individuals with high consumption of relevant foods were above the ADI. For residues of veterinary drugs, the MRLs in animal tissues are calculated by dividing the ADI by the total amounts of animal products that would be consumed by a high consumer.

The projected "market share" is not taken into account in the exposure estimates for additives because risk characterisation focuses on the possible risks to individuals, and some individuals may preferentially select foods containing the compound. Determinations of the intakes of pesticides, from residues on foods following agricultural use, have not taken into account the extent of use, or market share, of different pesticides used for treating the same pest. Because the residues occur randomly within the food supply, some recent assessments of the data from surveillance programs have used a probabilistic approach to determine the acceptability of residue levels.

In the case of exposure assessments for chemicals requiring pre-marketing approval, the pre-market estimates of consumption of foods containing the compound may be uncertain. The use of conservative assumptions at this stage to compensate for limited knowledge often leads to significant overestimation of exposure. Post-launch data collection, both on real-life use levels and actual consumption patterns, can help to increase the accuracy of the exposure assessments that were used in the initial risk characterisation. Real-life data obtained after marketing to correct these initial assumptions may indicate that it would be possible to remove some restrictions on use, but this may trigger the need for a revised characterisation of risk. Conversely, in the case of higher individual exposures than those initially predicted, perhaps due to a greater than expected success of a food/substance with consumers, restrictions on use could become necessary.

In some cases the risk characterisation considers only a very restricted number of specific uses, because the available toxicity data on the compound are sufficient to allow restricted food use only and a generally applicable guidance value, such as an ADI is not appropriate. An example of this is quinine, which is approved only for use in tonic water, and the projected intake can be matched closely to the available hazard characterisation data (Joint Expert Committee on Food Additives, 1993). A disadvantage of this form of advice is that any application for an additional use would require a reevaluation and a new risk characterisation.
5.2.2.3. Measurement of the concentrations of the chemical in foods: The concentrations of contaminants and other compounds that are found to be present in food need to be determined, prior to the risk characterisation process, by the collection of samples of relevant foods and their analysis for the compound. Attention needs to be paid to whether the sampling strategy and analytical techniques employed are adequate with respect to anticipated concentrations and frequency of occurrence of the substance in question. These aspects have been reviewed by Kroes and colleagues (2002).

An important problem that can influence exposure assessment of contaminants, and may lead to an unnecessarily conservative risk characterisation, relates to how data for samples that did not contain a measurable concentration are included in the exposure estimation. The failure to detect a measurable concentration, i.e. a "non-detect" result, means that the actual concentration could be anywhere between zero and the limit of quantification (LOQ). Different intake estimates will be obtained depending on whether it is assumed that all non-detects are zero, or $50 \%$ of the LOQ, or equal to the LOQ. This potential error in exposure assessment and therefore risk characterisation is especially important when the substance could occur in a wide range of different foods, but measurable concentrations are only rarely detected.

The concentration of a substance measured in a food is not always a true indication of what is available for absorption and uptake from the gastrointestinal tract. For example, substances that are tightly bound to the food matrix may not be released within the gut lumen or absorbed, whereas in other cases the food matrix may contain precursors of the compound that are converted to the compound during digestion and metabolism of the matrix, but are not detected by chemical analysis of the food for the compound per se. These difficulties can be resolved by suitably designed biomarker studies (see Section 5.2.4).

For compounds that undergo risk characterisation prior to approval, the intended use levels and concentrations in food (for an additive), or the anticipated residue levels (for a pesticide) are essential information; the associated risk characterisation, for example whether or not the projected intakes are below the ADI/ ARfD, relates to those uses and concentrations.

\subsubsection{Matching the duration of intake measurement to the toxicity of concern}

When a critical effect has been identified, the intake measurement should be appropriate for that endpoint. In most cases, the critical effect identified in hazard characterisation using animal experiments is observed following sub-chronic or chronic administration of the compound, whereas practicable intake estimations tend to be of short duration. In consequence, there is usually 
a mismatch when these two sets of information are integrated during risk characterisation.

Intake estimates based on dietary recall and/or food diaries are usually of relatively short duration $(24 \mathrm{~h}$ to 7 days) and, because of variability over time, tend to overestimate the long term intake by those who consume the substance and to underestimate the proportion of the population with intermittent intake (some of whom would be classified as consumers in a study of longer duration). Dietary history methods and food frequency methods are aimed at estimating long-term consumption patterns, but do not provide reliable estimates of individual intake. Short duration surveys emphasise extreme consumption based on contents in individual meals or due to seasonal effects: these peaks may be relevant when acute effects are of concern, but need to be put into perspective when long-term average intakes are the type of exposure relevant for the critical endpoint.

The NOAEL for an effect having a threshold in the dose-response relationship (see below) is usually lower after chronic administration than after short-term treatment; in consequence any mismatch during risk characterisation due to the use of short-term intake data and chronic hazard characterisation will normally lead to conservative advice. While this is not a problem if the resulting exposure estimates are below the human guidance value (e.g. an ADI), it may lead to unnecessary concern if risk characterisation indicates that the guidance value appears to have been exceeded. Recent developments in hazard characterisation have attempted to correct this anomaly by determination of a shortterm guidance value (ARfD) more relevant to the duration of high intake. This is discussed in detail below, under Section 5.3.3.1.

\subsubsection{The use of biomarkers to define the relationship between external and internal dose, and to bridge the gap between exposure assessment and hazard characterisation}

Biomarkers are biologically relevant measurements that can relate to intake (such as urinary excretion data), internal exposure (such as urinary excretion or plasma concentration data), effect (such as serum transaminases) or susceptibility (such as a genetic polymorphism). Such measurements can be used at different levels in risk characterisation, for example simply as a measure of exposure, or as a way of refining the considerations of interspecies difference and/or human variability in hazard characterisation. Ideally biomarkers would be used as a way of integrating the internal dose in potentially susceptible human subgroups under hazard characterisation with the internal dose measured during exposure assessment. Such an approach would be extremely data intensive and in reality is only likely to be possible for a small number of substances of the greatest concern to human health.
Biomarkers can provide important information for application of the "parallelogram approach". In this, data on in vitro biomarkers of the critical effect for the test species are compared with the in vivo response data for the test species, the difference is used to extrapolate from in vitro data for human preparations to the likely in vivo response in humans (see Eisenbrand et al., 2002).

Usually the food component itself or an appropriately defined metabolite serves as the biomarker of exposure. An understanding of the relationship between the biomarker and the fate of the substance in the body is essential, as is information on the dose-biomarker relationship. Biomarkers of exposure are usually intended to provide specific information about internal as opposed to external exposure. An advantage of biomarkers of exposure is that they take account of factors that may affect the bioavailability of a substance. They are usually specific to the particular chemical under evaluation, although in some cases the biomarker may be common to a number of different chemicals sharing common metabolic or biochemical properties. Many organophosphate pesticides, for example, share common urinary alkyl phosphate metabolites that have been used as biomarkers of exposure, but it should be noted that this may not reflect the differing potencies of organophosphates, so cannot be used as a biomarker of effect.

Biomarkers of exposure require validation before the magnitude of the biomarker in an individual can be converted with any degree of confidence into a level of exposure that can be compared with the guidance value/risk level. Validation should take into account the time course for defined exposures in relation to the biological sampling for the biomarker measurement. Ideally the dose-biomarker relationship should be ascertained and the impact of inter-individual variation on the dose-biomarker relationship should be characterised.

Biomarkers can also provide a useful bridge between exposure assessment and hazard characterisation in cases where other components present in the food matrix affect the bioavailability and hence the systemic dose of the substance under consideration. Examples include the enhancement of iron absorption by meat and ascorbic acid and retardation of iron absorption by milk or phytate (Scientific Committee for Food, 1993), or the faster absorption of vitamin A when given as a supplement compared with its absorption from liver (Buss et al., 1994). Thus, the presence and concentrations of other components known to enhance or retard absorption of the substance under consideration may need to be determined. More complex than understanding the possible interactions between two or several identified components can be the understanding of the impact of the overall food matrix, the number and spacing of meals and the nutritional status of the 
individual on the 'internal dose' and its effects. Such data, however, are rarely available. Biomarkers may also help where there are differences in bioavailability of the chemical when present in the human diet, compared with the conditions in the studies in humans or in animals that were used for hazard characterisation (e.g. studies in which the substance is given in solution by gavage); these can create problems of interpretation when these aspects are brought together in risk characterisation.

Some biomarkers of exposure that provide useful measurements related to the internal dose can also be used in hazard characterisation, for example to define interspecies differences in internal dose. Another use of biomarkers of internal dose can be to allow the rational use of hazard characterisation data that have been derived using a non-oral route of administration. The extent to which data following exposure by a non-oral route can be extrapolated to oral intake requires careful consideration, and non-oral data would normally only be used in the absence of adequate hazard characterisation data following oral dosage. However, for some substances, such as contaminants, non-oral data may be all that is available for some effects. It may therefore be necessary to derive a systemic guidance value for exposure to a substance via the food supply from non-oral data. Biomarkers of systemic exposure after oral and non-oral routes can be used for route-to-route correction, because they allow for differences in absorption or bioavailability. An alternative approach under hazard characterisation is the use of a physiologically based toxicokinetic (PBTK) model to predict the internal dose for each different route.

The use of biomarkers of response or effect in studies in healthy volunteers can give valuable information, providing, because of ethical considerations, that the response is both non-adverse and reversible. Biomarkers of early events should be related to, and ideally critical for, the development of the adverse effect (hazard) that is the basis for the risk assessment (Renwick and Walton, 2001). In vivo animal experimentation is an important step in the validation of such early event biomarkers in relation to the generation of the ultimate adverse effect. For example, measurement of the inhibition of plasma cholinesterase represents a biomarker for total exposure to organophosphate and carbamate pesticides, whereas inhibition of erythrocyte acetylcholinesterase is considered a biomarker of effect. It is important that the biomarker response occurs at similar or lower doses than would the adverse effect: such relationships can be addressed in special studies in animals.

\subsubsection{The influence of the nature of the hazard and hazard characterisation on risk characterisation} 5.2.5.1. Quality of the hazard database: The reliability of risk characterisation is dependent on the quality of the hazard database relevant to the issues raised in problem formulation.

Hazards may be identified from human studies, animal-based toxicology and in vitro toxicology, as well as being inferred by structure-activity considerations. Hazard characterisation has to address two main aspects for each endpoint:

- qualitative considerations of the importance and relevance of the health endpoints for humans, and

- quantitative considerations of the dose-response relationship and its application to the human population.

These aspects have been addressed in more detail in earlier FOSIE publications (Barlow et al., 2002; Dybing et al., 2002; Edler et al., 2002).

As experimental and epidemiological data are collected they may reveal additional issues, such as highly sensitive life-stages These need to be taken into account and may even become the focus of the advice to risk managers.

The importance of human data in hazard identification is well recognised. There are many advantages to using human data, for example the outcomes are those that humans will experience, the populations under study are usually exposed by the relevant route, namely ingestion, and the chemical exposures occur at concentrations present in the food supply. For these reasons such data usually take precedence over animal data. For some agents, hazards have been identified by epidemiological studies before they were detected in laboratory animals. The contribution of epidemiology to risk assessment has been reviewed in more detail in an earlier FOSIE publication (van den Brandt et al., 2002). However, human epidemiology findings are not always suitable for risk characterisation. The accuracy and precision of the exposure data in observational epidemiology studies are not always optimal, and the results can be affected by bias and confounding. In addition available epidemiology data may not be adequate, because adverse effects identified in animal studies were not investigated, or the duration of exposure, the life-stage exposed or the length of follow-up were insufficient for the hazard to be expressed. Therefore, although human data can provide the most secure advice, it has to be appreciated by both risk managers and all stakeholders that in most cases risk characterisation has to be based on other types of data, mostly from in vivo studies in animals.

Animal models continue to be the main system for hazard identification of low molecular weight food chemicals, see Barlow and colleagues (2002) for more extensive discussion. The advantages of in vivo studies in animals, compared with in vitro studies, are that a 
whole organism, with its inter-related metabolic, circulatory, hormonal, neural and immunological functions and organ systems intact, is the most appropriate model for humans, the dosage can be exaggerated compared with human exposures in order to increase sensitivity, and the substance can be administered under welldefined experimental conditions by the oral route.

Once all adverse effects associated with a substance have been identified, there is a need to determine which of these effects, usually termed the critical effect(s), might be most relevant to the predicted human exposure. This decision is based on the sensitivity of the target organ(s) and the relevance of the hazard to anticipated exposed populations. In addition, the hazard characterisation process needs to take into account the fact that some hazards, such as genotoxicity, are evaluated by low-dose extrapolation while other hazards may be characterised by the application of uncertainty factors (see below). It is important in risk characterisation to ensure that the advice to risk managers focuses on the effect(s) that could be produced at the lowest human exposures.

Historically, different approaches have been adopted for the risk characterisation of threshold and nonthreshold effects (Edler et al., 2002). In most cases, the approach for both begins with an analysis of the available dose-response data for the critical effect. This may or may not involve curve fitting, but the goal is to derive a value close to the bottom end of the observed doseresponse curve (the starting point for any extrapolation beyond the observable response range). Where the doseresponse data cover current human exposure levels from food then the data can be used without extrapolation outside the observed range. The aim is to predict risk in the range of human exposures, or to predict an exposure level considered to represent negligible risk.

\subsubsection{Threshold approaches in hazard characterisa-} tion: The hazard characterisation approach for threshold effects involves the derivation of a health-based guidance value, i.e. a level of exposure at or below which there would be no significant health risk if consumed daily throughout life, for example the ADI, TDI, or reference dose (RfD) or, over the period of one day or one meal, the ARfD. However this approach provides little advice to risk managers about any risks above the guidance value.

From a biological perspective a practical threshold in the dose-response relationship would represent a certain dose, or range of doses, above which there are substantial or biologically relevant changes that would affect the health of the organism and which would be considered adverse. Below this dose range, no biologically significant effects are expected due to homeostatic control processes. The presence of a threshold cannot be proven from experimental data, since the absence of a detectable effect at low doses could be either because the dose is below a threshold in the dose-response, or because the response is below the level that can be detected by the test system. In consequence, conclusions about the existence, or not, of a threshold are based on biological plausibility and expert judgement of the underlying biology of the test system.

All NOAELs, or other measures of potency/sensitivity (see Edler et al., 2002), from animal and other experimental studies are listed for consideration. The most sensitive of the relevant adverse effects, i.e. that occurring at the lowest dose relevant to the route and duration of exposure, becomes the focus for detailed consideration under hazard characterisation and is termed the "critical effect". The rationale for focusing on this effect derives from the assumption that any risk to humans from other effects detected at higher doses in animals would also occur at higher doses in humans and therefore a guidance value based on the critical effect will cover other adverse effects. The rationale for the selection of the critical effect should be described clearly as part of the risk characterisation, including supporting evidence on biological plausibility, the shape of the dose-response curve and the precision of the NOAEL. Where an effect that has been detected at low intakes is excluded because it is not considered adverse, or is not considered relevant to the risk characterisation that is being undertaken, this should be stated clearly, together with all information supporting this conclusion.

The different starting points that are developed (e.g. NOAEL, LOAEL or BMD) are used as indicative of an intake in the experimental study (animal or human) that would be close to the real biological threshold. Their strengths and weaknesses have been reviewed in an earlier FOSIE publication (Edler et al., 2002).

When a NOAEL can be derived from a study in humans that has shown relevant effects at higher doses, then this would be utilised in the derivation of guidance values or advice. The main issues regarding uncertainty in a NOAEL derived from an observational epidemiological study are the possible role of confounders and the precision of both the incidence and exposure data. For rare outcomes, only large studies with substantial numbers of persons would be suitable to identify an exposure-outcome relationship. The quality of the exposure data and estimates need to be scrutinised, to determine the confidence with which any dose-response relationship can be constructed. Uncertainty may be reduced if the results of several similar or related highquality epidemiological studies are combined in a metaanalysis to provide a reliable and statistically precise indication of the relationship between dose and effect.

The main problems with the use of either the NOAEL or the LOAEL are that these values are critically dependent on the study design, dose selection, group size and the precision with which the investigation is 
performed, and they are influenced little by the shape of the overall dose-response relationship. These criticisms can be avoided by the use of the BMD, advantages of which are that the full dose-response data are used to derive the value, and that use of the lower confidence interval will "reward" well-conducted studies, that have narrow confidence intervals, with a higher BMD. Although there is often no significant numerical difference between a NOAEL and a BMD, the former allows an easier or more facile explanation, because the starting point is a "safe" intake under the conditions of the experiment, whereas the BMD is estimated to be associated with a response. In reality, this is a difference in perception; because of its limitations the NOAEL does not necessarily represent an intake with zero risk of adverse effects under the study conditions, but simply an intake with no observed adverse effects. The outcomes of risk characterisations based on NOAELs are not greatly different from those based on BMDs that represent the lower confidence interval on the dosage giving a 5 or $10 \%$ response.

The starting point from the dose-response data, for example the NOAEL or BMD, is converted into a health-based guidance value, such as an ADI, by the use of uncertainty factors, which are applied to the external dose expressed on a $\mathrm{mg} / \mathrm{kg}$ body weight basis. The uncertainty factors that have been used for the past 40 years were developed by the FDA and adopted by the JECFA and are now accepted for the risk assessment of threshold effects. The usual default factors are 10 -fold for species differences (when the NOAEL is derived from a study in animals) and a further 10-fold to allow for human variation. These factors are a part of hazard characterisation, and are supported by scientific data. There have been a number of reviews of the use of the default uncertainty factors (see Edler et al., 2002), and questions raised about the appropriateness of using the same default factors, irrespective of the test species and the metabolic fate and effects of the compound. Chemical-specific data on toxicokinetics, physiologically-based toxicokinetic modelling, or mode of action can be used to replace part of the default factor with appropriate chemical-specific adjustment factors (CSAFs) (Renwick, 1993; World Health Organization, 1994; Edler et al., 2002), however, there have been few examples so far where appropriate data have been available.

When different adverse effects are considered during hazard characterisation, the different possible guidance values would be affected by both the sensitivity of the organism to each effect, as indicated by the NOAELs, LOAELs or BMDs, and the size of the uncertainty factor that would be applied to each effect. It is therefore necessary to take both the sensitivity and the uncertainty factor into account in an iterative fashion before finally selecting an overall critical effect for deriving a guidance value and formulating advice to risk managers. If a standard default uncertainty factor is applied to all effects then the critical effect would be that with the lowest NOAEL. Potential guidance values obtained using the different combinations of possible adverse effects and their respective uncertainty factors need to be compared and the most appropriate guidance value (usually the lowest of those meeting the relevant criteria) adopted. This is particularly important when both human and animal data are available, because the overall uncertainty factor applied to human data will usually be lower than that applied to data obtained from studies in experimental animals. As an example, consider a chemical that, in adequately conducted studies, produces histopathological effects on a target organ such as the heart in the rat, with a NOAEL of $1 \mathrm{mg} / \mathrm{kg}$ body weight per day and also produces mild neurobehavioural effects in human volunteers, with a NOAEL of $0.3 \mathrm{mg} / \mathrm{kg}$ body weight per day. Obviously, the human study could not provide comparable information on potential effects of the chemical on the target organ identified in the rat. A potential guidance value based on the critical effect in the human study, with a NOAEL of $0.3 \mathrm{mg} / \mathrm{kg}$ body weight per day and a default uncertainty factor of 10 -fold, would be $0.03 \mathrm{mg}$ / $\mathrm{kg}$ body weight per day. However, if the NOAEL from the rat study were used, with a default uncertainty factor of 100 -fold, this would give a potential guidance value of $0.01 \mathrm{mg} / \mathrm{kg}$ body weight per day. As there is no assurance that humans would not be sensitive to the cardiac effect observed in the rat, the guidance value would be established at $0.01 \mathrm{mg} / \mathrm{kg}$ body weight per day. Under such circumstances it is important that the advice to risk managers explains clearly why data from a study in rats has been used in preference to data from a human study.

\subsubsection{Non-threshold approaches in hazard character-} isation: The traditional position has been to consider that there may not be a threshold dose for effects involving genotoxicity. Thus, adverse health effects originating from genotoxic events, such as heritable disorders and some cancers, are generally assessed differently from non-genotoxic responses.

Three non-threshold approaches have been used historically in the formulation of advice for such effects:

- quantification of the risk at different levels of human exposure,

- ranking the hazards from different compounds producing similar hazards, but without quantification of the risk at human exposure levels, and

- recommendation that the exposure should be minimised, without any further use of either the exposure estimation or hazard characterisation. 
For non-threshold effects the starting point is usually a fixed response (e.g. a certain percentage tumour incidence) within but towards the lower end of the doseresponse curve, that is derived by fitting a mathematical model to the data (Lovell and Thomas, 1996; Dybing et al., 1997; Sanner et al., 2001; Van Landingham et al., 2001). The fixed response can be used directly to produce advice that allows comparisons to be made between different compounds producing the same response in the same species and strain of animals. Alternatively it can be the starting point for low-dose hazard characterisation, down to a fixed response such as a lifetime risk of one in a million or one in one hundred thousand (see Edler et al., 2002).

An important distinction between animal and epidemiological studies, which is particularly relevant to the detection of carcinogens, is that a 2-year rodent study yields estimates of lifetime risk at each dose level. In contrast, most epidemiological studies are partial-lifetime studies and do not involve sufficiently long follow-up to measure lifetime risks directly.

For non-threshold effects, the quantitative risk estimate can be calculated by extrapolation from a given incidence in experimental studies, usually about 10 or $25 \%$, down to a low predicted lifetime risk, such as one in hundred thousand $\left(10^{-5}\right)$ or one in a million $\left(10^{-6}\right)$. The selection of the starting point for extrapolation from the animal data (for example the external dose giving a $5 \%$ response) can be derived by modelling the dose-response data in the experimental range (Edler et al., 2002). In some risk assessments the external dose has been converted to a human equivalent target organ dose of the proximate carcinogen by the use of a PBTK model. However, such scientific sophistication has little impact on the resulting risk estimate, which is largely determined by the mathematical low-dose extrapolation model used. While determination of the starting point and selection of the mathematical model are part of hazard and risk characterisation, the final output is a quantitative estimate of risk, e.g. the intake associated with an increased lifetime risk of developing cancer of 1 in $10^{6}$ - sometimes termed the virtually safe dose (VSD). The acceptability of the estimated risk requires a risk management or societal view.

Because this method provides a quantitative estimate of the risks associated with specified exposures, it is essential that the limitations and uncertainties are clearly described under the risk characterisation advice that is given to risk managers. There are considerable uncertainties associated with the risk estimates derived by low-dose extrapolation of animal data down to a lifetime risk of 1 in $10^{5}$ or 1 in $10^{6}$. The dose-response relationships for biochemical and pathological changes over such a range are never known, and therefore default assumptions are necessary. Historically a number of mathematical models have been proposed for low-dose extrapolation (Lovell and Thomas, 1996), but these can give widely differing risk estimates, and it is rarely known if the mathematical model selected reflects the underlying biological processes for a particular substance. The debate on this issue continues (see for example Lutz, 1998; Zito, 2001). The simplest and most readily applicable assumption is that there is a direct linear relationship between dose and biological response. This approach is being used increasingly because it is robust and readily applicable to a wide variety of databases. However, it is increasingly questionable given our developing understanding and knowledge about cytoprotective mechanisms and DNA repair processes which could reverse any low-level genotoxic effects. Non-linearity in the dose-response relationship could arise at the high doses used in the animal studies due to saturation of a detoxication or cytoprotective process (which would result in a steeper doseresponse relationship at high doses) or saturation of a bioactivation process (which would reduce the slope at high doses). Non-linearity can also arise from interactions with other factors not present in experimental conditions, but occurring widely in the human realworld situation. Synergistic effects could increase the slope at all or only some dose levels; antagonistic effects could have the opposite effect, reducing the slope at all or only some dose levels. The uncertainties arising from the mathematical extrapolation process are considerably greater than the errors or uncertainties in the estimation of the starting point, and it is important that the limitations and assumptions are provided as part of risk characterisation.

Despite these uncertainties, the advice to risk managers from low-dose extrapolation can appear to offer mathematical precision. For example, hazard characterisation may give an upper bound risk estimate of 1 in $10^{6}$ for a daily intake of $3 \mathrm{mg}$; in reality, the risk estimate associated with a daily intake of $3 \mathrm{mg}$ could be from zero to 1 in $10^{6}$, but both risk managers and other stakeholders tend to interpret the upper bound of the risk estimate as if it were the central tendency. The advice from risk characterisation should be sufficiently transparent that risk management decisions can take into account the imprecision and conservatism built into the estimation of risk.

A form of advice that avoids the uncertainties inherent in low dose extrapolation, but which can be useful to risk managers in the prioritisation of problems, is to give a value that can be used to rank a series of compounds that exhibit the same biological properties. For this approach the starting point can be the direct comparison of the potency of chemicals sharing common hazards, e.g. are both genotoxic and carcinogenic. As a part of risk characterisation the ratio of the human exposure to a common point on the dose-response relationship can be used to indicate the relative (but not 
absolute) magnitude of the risk for that substance compared with others that share similar properties, i.e. an exposure-potency index. Although low dose extrapolation is not an explicit part of such an approach to risk characterisation, the use of such information by risk managers requires an assumption that there is no difference between compounds in the linearity, or the extent of any non-linearity, in extrapolating from the relative potency estimated from the animal study, down to the predicted human exposure considered by the risk manager.

The other advice that is sometimes given to risk managers for compounds that show properties such as genotoxicity is that the exposure should be reduced to as low as reasonably achievable or practicable (ALARA or ALARP). This approach does not run the chance of over-interpreting the hazard characterisation data and leaves it to the risk manager to decide the level of reduction that may be technologically and financially practicable. However, it provides no practical advice to risk managers and the same advice would be given for compounds with high and low potencies. In contrast, an exposure-potency index or low-dose extrapolation, despite the limitations and assumptions, allows risk managers to prioritise problems.

\subsection{The nature of advice to risk managers}

The advice to risk managers may be quantitative or qualitative, dependent on problem formulation, data availability and the nature of the compound or substance.

Quantitative advice can be in one of the following forms:

- an estimate of the risks associate with different levels of exposure (e.g. for an unavoidable contaminant);

- a health-based guidance value (e.g. for an additive, pesticide, veterinary drug or other avoidable contaminant);

- as a ratio between the NOAEL in experimental or epidemiological studies, and the estimated amount of human intake/exposure, referred to as a "margin of exposure";

- as a ratio between of the NOAEL and the actual amount of human intake/exposure, referred to as a "margin of safety" or a "safety margin";

- as recommended minimum and maximum intakes (e.g. for vitamins, minerals and other nutrients).

Qualitative advice can be in the following forms:

- a classification of "ADI not specified" may be allocated (e.g. for an additive when its addition to food under good manufacturing practice does not need to be restricted, because of the absence of any evidence of toxicity even at high dose levels);

- approval may be use-specific, that is, the substance is considered safe in the context of the intake that is estimated from a certain specified use or uses (e.g. a vitamin, mineral or additive);

- to avoid certain foods (e.g. aflatoxin-contaminated nuts from certain sources);

- to avoid certain processes (e.g. use of ethylene oxide for sterilisation of spices);

- to modify production processes (e.g. use of a different production method for hydrolysed vegetable protein to avoid formation of chloropropanols);

- to minimise exposure to as low as reasonably achievable (e.g. for unavoidable toxic contaminants);

- to reduce intakes (e.g. of certain types of fat);

- to reduce or avoid intakes by certain sectors of the population (e.g. liver and vitamin A supplements by pregnant women).

Any advice to risk managers should clearly explain any key assumptions and the nature and magnitude of any uncertainties in the risk characterisation. This applies whether the assessment is based on epidemiology or animal data and whether the effect is considered to be a threshold or non-threshold effect. Where there might be a range of options for risk management, the advice should point out any scientific basis there might be for pursuing one option rather than another.

\subsubsection{Advice concerning individuals at increased risk}

Risk characterisation should include information not only on the general population, but also on any subpopulations considered to be potentially susceptible. "Potentially susceptible" in this context includes those who may be at greater risk of adverse effects because their particular dietary pattern results in greater intake, and those who may be at greater risk because their underlying physiological condition (e.g. life-stage or disease) or a specific genetic factor results in a greater internal dose or greater sensitivity.

If particular subgroups are at risk then advice should focus on what is known about exposure/intakes in those subgroups and identify any major information gaps. In cases where the risk assessment indicates that the general population or certain subgroups may exceed a health-based guidance value, then risk managers may need much more detailed information about the characteristics of the exposure/intake distribution. Specific advice may be recommended for subgroups of the population when those individuals can recognise that the advice relates to them (e.g. avoidance of aspartame 
for people with phenylketonuria, or reduction in consumption of liver and vitamin A-only supplements by pregnant women). Other potentially susceptible subgroups and individuals, who cannot be given usable and meaningful specific advice, should be regarded as part of the general population, and taken into account in the risk characterisation for the general population.

\subsubsection{Consideration of individuals with high intakes:} Risk characterisation considers both individuals with average exposures and those with high exposures. High exposure may be related to life-stage, cultural aspects and/or qualitative and/or quantitative food preferences. Definition of individuals with high consumption of food chemicals may be especially important when the hazard characterisation process identifies potentially sensitive populations that should be investigated in exposure studies, such as those that can be recognised by sex or age, or by pre-existing conditions (diabetes) or dietary habits (dieters, vegetarians, etc.).

The methods mainly used to determine individual intakes are based either on point estimates, "simple" distributions, or probabilistic analyses. Each of these approaches has its shortcomings but also its advantages.

Point estimates use single values for the amount of food consumed and the concentration in the food of the chemical in question. Simple distributions take account of variation in consumption of the relevant food items, but assume that the chemical in question is present in all foods at a defined maximum level (e.g. the maximum permitted level for a food additive). Point estimates and simple distributional approaches tend to use 'worst case' assumptions without taking into account the frequency of occurrence of a chemical, the variation in concentrations of the chemical in different foods, or the variation in food consumption, and therefore normally overestimate exposure.

Both "worst-case point estimates" and "worst-case simple distributions", which assume that individuals with high consumption of food chemicals not only eat high amounts of the relevant foods but also that they are always, or at least predominantly, exposed to products containing a high concentration of the substance in question, are clearly unrealistic. Continuous high consumption of high concentrations happens only rarely in practice, and for most substances, the individuals with high consumption of relevant foods are exposed to a normal distribution of concentrations of the chemical under assessment. Exceptions to this include dietary supplements and other foods with instructions for consumption, and the regular consumption of an unusually contaminated food supply. Point estimates and simple distributions that assume individuals with high consumption of relevant foods are predominantly exposed to the mean concentration of the substance in question will generally overcome this particular problem.

The definition of the intake by a "high consumer" is usually the intake at the 90th, 95th or 97.5th percentile of the intake distribution. The percentile selected is primarily a risk management decision and will be influenced by consideration of a number of issues. These include the length of the tail of the distribution curve, whether the tail comprises an identifiable subgroup (e.g. children or an ethnic group), and whether the critical effect used to derive the health-based guidance value is mild or severe in nature. The decision is taken on the understanding that health-based guidance values are intended for people within the normal range of cultures and lifestyles and that the definition of a "high consumer" would not normally be expected to include those with bizarre food preferences or habits. It should also be borne in mind that when higher percentiles are selected, the precision of the value is critically dependent on the size of the database from which it is derived.

A major difficulty arises in calculating the high consumer intake when a chemical is distributed widely in the food supply. The high intake from one food can be calculated as the concentration present multiplied by the consumption of that food by a high consumer. Theoretically the total intake for a high consumer could be calculated by the addition of the resulting high intakes for all relevant foods, but this would grossly overestimate possible intake, because the same individual would not be a high consumer for all relevant foods. This difficulty can be overcome by the use of real intake data based on food diaries, market basket methods or biomarkers of exposure, or by the use of probabilistic methods which allow for alternative selection choices. Where such approaches may not be possible, an alternative strategy is to place a limit on the number of foods from which high consumer intakes may be summed, for example two or three foods only, then adding to these the calculated average intakes from all remaining foods.

Probabilistic approaches model the distribution of both the consumption of the relevant foods and the presence and concentrations in the foods of the chemical to be assessed. Such approaches require sufficient measurements of the concentrations present in foods and the availability of food consumption databases to make the assessments meaningful. In many cases, the statutory requirements for monitoring do not allow characterisation of distributions in a statistically meaningful way. When scientifically sampled data are available, such models can be used to estimate the likelihood that a defined intake level for a substance will be exceeded. Providing that the available data are of sufficient quality, probabilistic approaches are potentially the most suitable methods to combine the concentrations of the substance to be assessed in the foods with the consumption of the foods that actually contain it, thereby 
providing a basis for realistic exposure assessment. However, these methods for the estimation of exposure require further refinement, including a quantitative measure of uncertainty.

When distinct groups of the population are identified as having high intakes, the risk characterisation usually focuses on that group(s). For example, infants may have higher intakes of a particular substance because breast milk or infant formula is the sole source of nutrition in the first few months of life. Similarly, consumption of juices and fruits can be high in young children. Another, and more general reason for higher intakes by infants and children, is because of their higher calorie intake relative to their body weight. In Europe and in the WHO advisory bodies, the higher potential intake is taken into account under exposure assessment and the higher potential target organ sensitivity under hazard characterisation, i.e. prior to their integration into risk characterisation. In the USA the Food Quality Protection Act requires the use of an extra 10-fold uncertainty factor for pesticides in the case of infants and children because of their higher intakes and greater potential susceptibility, unless there are comprehensive hazard characterisation data available for the relevant age group (Environmental Protection Agency, 1999a). From a European Union (EU) perspective the United States legislation applies the uncertainty factor as part of risk characterisation, in contrast to other uncertainty factors that are applied during hazard characterisation, as part of the derivation of the health-based guidance value. The formulation of advice to risk managers should take into account those groups with highest intakes and those predicted to have the greatest susceptibility, looking at the strengths, limitations and uncertainties inherent in both aspects.

\subsubsection{Consideration of individuals with high suscept-} ibility: The dose-response relationship from studies in small groups of animals or small groups of human volunteers would be unlikely to be representative of the relationship in the widely diverse human population. Inter-individual differences in the toxicokinetics are an important source of variability in response. Such variability may arise from differences in genetic constitution and environmental influences including diet, nutritional status, physiological states, such as pregnancy, and pathophysiological states. Data will only rarely be available to address human variability in toxicokinetics, but if so, the issue needs to be considered during hazard characterisation.

The default uncertainty factor of 10 for inter-individual human variability, which allows for both toxicokinetic and toxicodynamic differences, can be subdivided into kinetic (3.16 or $\left.10^{0.5}\right)$ and dynamic $(3.16$ or $10^{0.5}$ ) components (World Health Organization, 1994). There is wide inter-individual variation in polymorphic pathways of metabolism, for example CYP2D6, CYP2C19 and N-acetyl transferase-2 (Dorne et al., 2002 and 2003; Knudsen et al., 2001; Vineis et al., 2001). Differences between extensive metabolisers (EM) and poor metabolisers (PM) for substrates of CYP2D6 and CYP2C19 can greatly exceed the default uncertainty factor of 3.16 or $10^{0.5}$, which normally allows for human variability in kinetics (Dorne et al., 2002 and 2003). In the case of CYP2D6 the difference in internal dose between EM and PM subjects may exceed 30-fold if the substance is metabolised exclusively by CYP2D6, but the difference would be only about 2-fold if CYP2D6 was responsible for the elimination of $10 \%$ or less of the compound (Dorne et al., 2002). Replacement of the default factor of 3.16 by a CSAF (see Edler et al., 2002) for human variability requires selection of a percentage of the population that the CSAF would cover. This is primarily a risk management decision, and is analogous to selecting a point estimate, such as the 95 th or 97.5 th percentile on the intake distribution curve to represent a "high consumer". When a CSAF is calculated, the uncertainty related to the validity of the default for the population would be replaced by uncertainty for the individual, arising from human variability.

Increased susceptibility associated with different lifestages is taken into account as part of hazard characterisation by experimental studies in animals throughout different life-stages. Only rarely are there data directly addressing the issue of human variability in toxicodynamics, because of obvious ethical considerations, and because the aim of hazard characterisation usually is to establish exposures that would be without adverse effect in humans. However, knowledge of the mode of action of the chemical in animals combined with general physiological and biochemical understanding, and/or in vitro data on the chemical itself, could be used to assess likely human variability in toxicodynamics. Biomarker data from studies in humans could provide valuable information on variability in a reversible and/or a non-adverse response if measured in a controlled clinical study, or for irreversible effects if measured as part of an observational epidemiology study, although such data will be influenced by variability in exposure.

\subsubsection{Advice related to simultaneous exposure to different chemicals in food}

Studies undertaken for hazard identification and characterisation usually investigate the chemical in isolation, and not in combination with other substances to which humans may be exposed at the same time. However, food represents an extremely complex mixture of substances, which includes nutrient and non-nutrient chemicals that are an inherent part of the organism from which the food is derived, contaminants that occur 
due the environment in which the food was grown, chemicals that arise as a result of processing and heat treatment, as well as man made chemicals, such as food additives and pesticide residues.

\subsubsection{Ways in which chemicals may interact: In recent} years, public concern has focused on possible effects arising from exposure to multiple chemicals in foods, sometimes termed the "cocktail effect". Recent concerns were fuelled by publicity surrounding a publication (Arnold et al., 1996) that reported large synergistic interactions between combinations of xenoestrogens, the magnitude of which was such as to raise questions about the validity of risk characterisation based on data for single substances. However, the data could not be replicated by the original study authors nor by others (Ashby et al., 1997; Wade et al., 1997) and the report has since been withdrawn (McLachlan, 1997). However, because substances are investigated as single entities, and not in combination, this has raised a legitimate question that needs to be considered as part of risk characterisation. Most attention in the media has been on combined exposures to man-made substances, but risk characterisation needs to take a broader view and consider whether other substances in food could act in combination with the substance under evaluation to influence safety. Any interaction between the substance and the food matrix would be investigated under hazard characterisation, to determine if this affected either the absorption or bioavailability of the substance, or its potential for toxicity. Incorporation of any substance into the diet of test species should allow for both combined effects and interactions between the substance and the rest of the diet. Such interactions may represent a hazard of potential concern for humans, for example the impaired absorption of fat-soluble vitamins associated with the fat replacer, "Olestra", or the impaired absorption of minerals in the presence of dietary fibre. However, expert judgement is essential because incorporation of the test substance at high levels into the diet of test animals may perturb the animals' nutrition and produce effects that are irrelevant at the predicted or known human exposure levels.

In principle, chemicals present in food could exhibit combined effects, such as joint similar or joint dissimilar action, but these would not be described as interactions because one substance does not alter the activity of the other (see Dybing et al., 2002 for further explanation). Interactions arise when one chemical alters the degree and possibly also the nature of the potential effects of other individual food chemicals consumed around the same time. Combined intake can arise when the different substances are in the same food, in different foods consumed in the same meal, or even different foods consumed within a short time frame, such as a period of a few hours or days. The time-base that needs to be considered relates to the time over which the different chemicals may be present in the body and could produce a toxicokinetic interaction, and the duration of any possible biological effects, which could produce a toxicodynamic interaction. A toxicokinetic interaction would be where one chemical alters the kinetics of a second compound, for example by altering its absorption or by inhibiting its metabolism. A toxicodynamic interaction would be related to the mode of action, and could occur for example if one chemical interfered with a cytoprotective process thereby increasing sensitivity to another chemical.

Phenomena such as antagonism and synergism generally are seen only at exposure levels above the effect levels for the individual chemicals (Groten et al., 1997). There is little likelihood that such interactions would occur for the vast majority of man-made chemicals in food because risk characterisation, based on NOAELs and uncertainty factors, aims to ensure that the intake of each individual chemical would be without significant effects. However, in cases where chemicals have the same mode of action on a common target, then concentration addition applies, and effects could be produced, even when the concentrations of each individual chemical is below its no-effect level (Jonker et al., 1996; Tajima et al., 2002), particularly when there may be simultaneous exposure to a large number of chemicals that share a common adverse effect, such as xenoestrogenicity (Rajapakse et al., 2001). Therefore attention needs to be focused during risk characterisation on substances that share a common mode of action.

Toxicity studies have only rarely investigated mixtures of chemicals and there are problems of interpretation when adverse effects are detected. Although the available scientific studies on interactions between substances do not support the public concerns (Committee on Toxicity of Chemicals in Foods, 2002), there are a number of circumstances where combined exposure should be taken into account as part of risk characterisation. Risk characterisation of combinations of chemicals is based on an understanding of metabolic or mechanistic considerations, rather than on direct experimentation. This has been the approach adopted for food additives in the establishment of group ADIs and for some contaminants in the establishment of toxic equivalency factors for combining intakes of structural analogues.

5.3.2.2. Additives: A group ADI may be set where additives display similar toxic effects, providing that the additives are in the same range of toxic potency; the group ADI might be based on the average NOAEL or the lowest NOAEL of different members of the group (World Health Organization, 1987). Historically group ADIs have been set for compounds that are close structural analogues, or that share a common potentially toxic metabolite, such as the allyl esters. Additives that are not 
close structural analogues could show joint similar or joint dissimilar action dependent on mode of action and exposure level. As discussed above, joint dissimilar actions or toxicokinetic interactions would be unlikely, even in sensitive individuals providing that the intakes were below their respective ADI values. In contrast, joint similar action could result in dose addition and an increased risk, if a number of additives shared a common mode of action. Historically, this has not been considered systematically in the sequential setting of ADI values for different food chemicals by advisory bodies. A review of the adverse effects produced by approved food additives with numerical ADI values has concluded that this is not a significant concern (Groten et al., 2000).

5.3.2.3. Pesticides residues: The presence of multiple pesticide residues on food crops has raised public concern and has triggered work both in the USA and the UK to look at the potential effects of pesticide mixtures (Environmental Protection Agency, 2001 and 2002; Committee on Toxicity, 2002). Many of the reported interactions between pesticides have been of a toxicokinetic nature, arising from the high doses used in the interaction study. Toxicokinetic interactions can occur when compounds compete for the same enzyme or process, and are present in such concentrations that the process becomes saturated. Another type of interaction is when one compound increases the amounts of an enzyme (induction) and thereby increases the rate of metabolism of a second compound. This could be toxicologically important if the enzyme bioactivates the second compound, but again would occur only at doses high enough to induce the enzyme. While such interactions are possible in high dose animal studies they are very unlikely to occur at the much lower doses to which humans are exposed, for example arising from multiple pesticide residues on foods. The reason for this is that foreign compounds are usually metabolised by highcapacity enzymes and there is normally sufficient enzyme available to metabolise both substrates, as if the other one (or more) compounds were not present. The review by the Committee on Toxicity (2002) concluded that although several studies on mixtures of pesticides have reported synergistic interactions, most had been inadequately designed and that the interpretation of the data had been based on an incomplete understanding of the concepts. Nevertheless, there were data from a few studies, which used high concentrations, that supported either synergistic or antagonistic interactions.

Hitherto, combinations of multiple pesticides have not been explicitly considered on a routine basis, despite the fact that some, such as organophosphates and carbamates, share a common mode of action and a joint similar action is to be anticipated. The potential for combined effects to be produced from exposure to a mixture of pesticides that share a common mode of action (joint similar action) has stimulated recent initiatives to consider risks from both aggregate exposure and cumulative exposure (Environmental Protection Agency, 2001, 2002). The environmental protection agency has defined aggregate exposure and aggregate risk as the combined exposure/risk for a single compound via any route and from all sources, including foods, drinking water, consumer products, as well as occupational and environmental sources - air, soil, water, indoor surfaces, etc. (Environmental Protection Agency, 2001). In cases where there is exposure from other sources, it may be critical, for example, to determine the relative intake from foods for individuals who are also occupationally exposed to a substance in an industrial or agricultural setting. While for someone regularly handling a pesticide, the relative contribution from residues in foods may be comparatively low, it could still be relevant, either due to an incremental increase in total exposure, or due to differences in the relevance for risk of different routes of exposure (oral, dermal, inhalation). Co-exposure from other non-food consumer products, such as home and garden pesticides, cosmetics and household-products, may similarly be relevant.

Cumulative exposure and cumulative risk represent the exposure/risk from total exposure to substances with a common mechanism of toxicity, irrespective of their source or route of exposure (Environmental Protection Agency, 2002). Pesticides exhibiting anticholinesterase activity have now been subject to cumulative risk assessment by the Environmental Protection Agency (2002). In order to conduct such evaluations, the individual exposure to each component contributing to the cumulative exposure must be known and the estimate adjusted to allow for differences in relative potency before the exposures are summed. These analyses are complex and require the establishment of toxic equivalency factors (TEFs) for related compounds and the adoption of probabilistic exposure estimates, in order to avoid combining deterministic worst-case exposure estimates for each pesticide into a totally unrealistic risk characterisation.

5.3.2.4. Contaminants: Polychlorinated dibenzodioxins (PCDDs), dibenzofurans (PCDFs), and certain PCB congeners are ubiquitous contaminants which share a common mode of action. The potential for joint similar action is taken into account as part of risk characterisation by the use of toxic equivalency factors (TEFs). The health-based guidance value, derived under hazard characterisation is based on the extensive toxicity database on 2,3,7,8-tetrachlorodibenzo- $p$-dioxin $(2,3,7,8$ TCDD), which is the most toxic and extensively studied member. The toxic potency of each congener is calculated by comparison with 2,3,7,8-TCDD. Risk characterisation involves determination of the intake of each 
congener, which is multiplied by its TEF, and the combined intake of all individual compounds, expressed in 2,3,7,8-TCDD equivalents, is then compared with the health-based guidance value for the group (Scientific Committee on Food, 2000b). Whereas there are internationally established methods for combining the intakes of the 2,3,7,8-halogenated dibenzodioxins, dibenzofurans and related analogues based on the use of toxic-equivalency factors, similar approaches are still under discussion for other food chemicals that may share a common mechanism of toxicity, for example polycyclic aromatic hydrocarbons.

\subsubsection{Micronutrients: An area of food safety where} there is a potential for significant toxicokinetic interactions, but which has received limited consideration relates to high doses of minerals in food supplements. Unlike low-molecular-weight foreign compounds, which have received considerable attention, and which usually cross cell membranes by passive diffusion and are metabolised by enzymes with high-capacity and lowspecificity, vitamins and minerals show homeostatic mechanisms which are based on low-capacity transporters and enzymes, which may be involved in the homeostasis of more than one micronutrient. With such highspecificity and low-capacity processes, a larger than normal intake of one micronutrient might significantly affect the absorption or plasma and tissue levels of another nutrient.

An unusual possible interaction has come to light for one dietary component, beta-carotene. While beta-carotene is not thought to be a carcinogen, it has been identified as possibly associated with an increase in the risk of lung cancer in smokers taking it in the form of dietary supplements (Alpha-Tocopherol, Beta Carotene Cancer Prevention Study Group, 1994; Omenn et al., 1996). In the occupational field it is notable that occupational carcinogens, such as benzene and arsenic, have primarily been identified in populations with high levels of smoking, while the increases in risk among nonsmokers have been of lower magnitude. Whether the activity of food-associated co-carcinogens would be expressed primarily in smokers would be of interest to those designing food-related epidemiology studies and also to risk managers.

\subsubsection{Advice related to different duration and patterns of human exposure}

Guidance values generated during hazard characterisation are usually derived from chronic bioassays or multigeneration studies performed in animals, and therefore relate to chronic human exposure. Whilst the $\mathrm{ADI}$ or TDI is the most appropriate guidance value for food additives and contaminants that may be widespread and relatively constant constituents of the human diet, it is of less value where the exposure to the chemical is either episodic or highly variable and the compound shows acute toxicity.

The patterns of human exposure to chemicals in food may be chronic (usually low-level), short-term (often at higher levels) or chronic low-level with occasional higher intakes. Short-term studies often only show effects at higher doses than those producing effects during chronic treatment. In addition, the nature of the effect may differ qualitatively, for example changes during short-term treatment may be precursors of chronic effects or may be acute effects to which tolerance develops during chronic treatment. Therefore guidance values related to chronic, or lifetime exposure to the chemical, for example the ADI, may not be appropriate for risk characterisation for short-term intakes of a compound, such as a pesticide. Because the doses required to cause adverse effects following acute exposure are usually higher than those causing chronic toxicity, the acute-NOAEL and health-based guidance value for acute exposure, would be higher than a guidance value, such as an ADI, based on a chronicNOAEL.

5.3.3.1. Use of an acute reference dose: The need for an alternative form of hazard characterisation to relate to short-term human exposures arose primarily from the recognition of the variability in residue levels of pesticides within agricultural crops. Surveillance programmes generally measure the residue level in a composite sample of a crop, but it is now recognised that there can be significant variation from unit to unit (for example, carrot to carrot) within a particular batch of a commodity. Thus, there may be a short-term high intake over one day or even a single meal. This would give a short-term peak of internal dose, and could theoretically give an acute effect, but such high-level exposure would not occur repeatedly, and there would be no increase in the steady-state body burden that would normally be associated with exposure to that chemical. Whilst it is logical to compare the potential intake arising from average residue levels, i.e. that present in the composite sample, with the guidance value related to chronic exposure, i.e. the ADI, it would be overly conservative to compare the potential intake at the highest residue level in a particular unit (e.g. one carrot) with such a guidance value, because high-level exposure would not occur repeatedly (Renwick, 2002). This has been the stimulus for short-term guidance values and has given rise to the concept of the acute reference dose or ARfD.

The ARfD is used in risk characterisation of exposures that are predicted to be both short-term and for effects that occur following acute exposure and are predicted to be reversible, e.g. inhibition of acetylcholinesterase by organophosphate and carbamate pesticides. Several regulatory bodies (Pesticides Safety 
Directorate, 2001; Codex Committee on Pesticide Residues, 2001) have stated that the need for an ARfD will be considered for all pesticides in the future, and the estimated short-term intake of pesticide residues will be compared with the ARfD in order to interpret the possible risks associated with unit-to-unit variability in residue levels.

The ARfD can be derived in a manner analogous to the ADI, but based on short-term effects. Acute toxicity tests, as currently performed, are usually only of value for identifying severe acute effects at high dose levels; they are of little value in hazard characterisation for food chemicals and components because the studies are not usually designed to establish NOAELs related to short-term exposure. In most cases the ARfD is calculated by dividing the NOAEL for the critical effect in the short-term study by an appropriate uncertainty factor (usually the default value of 100). Where the effect is dependent on peak plasma concentration $\left(C_{\max }\right)$, rather than the total exposure, as estimated by the area under the plasma concentration time curve (AUC), consideration should be given to reducing the uncertainty factor, as there are often smaller interspecies differences in $C_{\max }$ than in AUC (Renwick, 2000).

Recommendations for appropriate toxicological studies have not been fully developed for the determination of ARfDs, and therefore the values calculated under hazard characterisation for different compounds may be associated with different types and extents of uncertainty. If suitable studies of short duration of exposure are not available it may be necessary to use longer-term studies to derive an ARfD. Under these circumstances, hazard characterisation needs to consider the biological plausibility of the effect occurring after short-term exposure (defined as over $24 \mathrm{~h}$ ). For example, haemolysis observed in a 28 -day study would be considered a possible acute effect in the absence of information to the contrary, whereas fibrotic damage in an organ would not. In some cases, specific studies may need to be undertaken to characterise potential acute effects. The use of a specific study protocol would be of value in cases where there is concern over short-term, high level exposures. Suitable experimental protocols, which involve acute, sub-lethal exposure of test animals followed by detailed observational, biochemical and histopathological follow-up for 1-2 weeks are currently under discussion.

Risk characterisation may indicate the need for ARfDs for compounds other than pesticides if the exposure data show short-term or episodic peaks. Reasons for high levels of intake may differ with the type of food chemical. For example the intake of an additive can be influenced by brand selection, the intake of a macronutrient can be influenced by individual food preferences, and the intake of a contaminant will depend on how widely it is distributed within the food supply and whether individuals consume locally grown or nationally produced foods. In some cases, short-term peaks for exposure may be intentional on the part of the consumer, for example a very high intake of micronutrients from high dose supplements may be taken to prevent or treat a short-term condition, such as the use of vitamin $\mathrm{C}$ to prevent a cold.

Neither the ADI nor the ARfD address the possibly increased risks of chronic adverse effects associated with long-term low-level exposure, combined with occasional peak exposures. Any increase in risk has generally been handled by averaging the long-term low-level exposure combined with the occasional peak exposure. Such averaging makes the assumption that peak exposure is not important and exposure averaged over the lifetime is the major determinant of risk. Peak exposures on top of low-level continuous exposure could be particularly relevant to some hazards and some life-stages, for example exposure during embryonic development, and this is taken into account in setting the ARfD. This possibility emphasises the importance of integrating hazard characterisation and the exposure scenario within risk characterisation.

5.3.3.2. Compounds with very long half-lives: Although considerable attention has focussed recently on the problems of high level but short-term exposure and the development of ARfDs, a similar mismatch may occur under risk characterisation when an ADI is used for compounds with very long half-lives. Compounds such as 2,3,7,8-TCDD accumulate in the body and chronic toxicity correlates with the body burden at steady-state. Periods of above average dietary intake that represent only a small fraction of the half-life may not significantly affect body burden (this is discussed further in Section 5.3.4), and therefore both hazard characterisation and exposure assessment should relate to longer, toxicologically relevant, periods. The half-life of 2,3,7,8TCDD in humans is about 7.6 years and, in recognition of the need for risk characterisation to consider longterm average intakes, the fifty-seventh meeting of the JECFA established a provisional tolerable monthly intake of $70 \mathrm{pg} / \mathrm{kg}$ body weight (World Health Organization, 2002). In contrast, the SCF established a tolerable weekly intake of $14 \mathrm{pg} / \mathrm{kg}$ body weight, (Scientific Committee on Foods, 2001), while the UK committee on toxicity of chemicals in food, consumer products and the environment (COT) calculated a tolerable daily intake of $2 \mathrm{pg} / \mathrm{kg}$ body weight (Committee on Toxicity of Chemicals in Food, Consumer Products and the Environment, 2001). The hazard characterisation was similar in all three cases, and the apparent discrepancies arise from the time base over which the exposure was to be expressed, with the JECFA taking a long-term view, the European commission's scientific committee on food (EC SCF) using the usual period for a contaminant that 
accumulates, and the COT using a daily basis because that is the way that intakes are usually expressed. The COT considered that a TDI would be more transparent, and then, for clarification, indicated that occasional exceedance of the TDI would not be expected to result in harmful effects. These different outputs from hazard characterisation need to be interpreted under risk characterisation in relation to intakes averaged over a long period of time, i.e. weeks or months. Each method of expressing the guidance value needs to be interpreted carefully, and adequate advice is an essential part of both hazard characterisation and risk characterisation. In reality, apart from intake by pregnant women and by babies during breast feeding, the issue for dioxins is not short-term high level exposures, but the fact that the long-term intakes by a significant proportion of the population exceed the health-based guidance value. Dioxins illustrate the need for some quantitative form of hazard characterisation for threshold effects to give an estimate of the risk at intakes above the health-based guidance value (see next section).

\subsubsection{Advice when human exposure exceeds the health-based guidance value}

Health-based guidance values (such as the ADI for threshold effects or the dose associated with a calculated low lifetime risk such as one in $10^{6}$ for non-threshold effects) are estimates of intakes that could be considered to be without significant adverse health effects. Therefore, by definition, intakes that exceed such guidance values cannot necessarily be assumed to be without adverse health effects and there is a need to provide advice to risk managers, firstly on whether there are likely to be any increased risks to health and, if there are, the possible nature and magnitude of any increase.

When considering this issue the JECFA stated "Because in most cases, data are extrapolated from lifetime animal studies, the ADI relates to life-time use and provides a margin of safety large enough for toxicologists not to be particularly concerned about short-term use at exposure levels exceeding the ADI, providing that the average intake over longer periods of time does not exceed it." (World Health Organization, 1987). The JECFA did not give further details and clearly the duration of excessive exposure would need to consider the toxicokinetics of the compound and the mechanism or mode of action. The significance of a period of intake above the ADI is difficult to assess, and depends on both the magnitude and the duration of excessive intake, and whether toxicity is associated with the peak concentration or the average concentration. For chronic toxicity, the duration of excessive intake has to be considered in relation to the half-life of the compound and the associated body burden at steady-state. In the case of 2,3,7,8-TCDD, which has a very long half-life (see discussion above), a short period of high dietary intake may not produce a major change in body burden (Renwick, 1999b; Vrijens et al., 2002; World Health Organisation, 2002). Similarly, a short period of intake in relation to the time-course for toxicity may be insufficient to produce any effect, even if continued exposure at the same dosage would result in toxicological changes. This would be applicable, for example, to a shortterm intake of a high level of cadmium from food. Interpretation is more complex in the case of dioxins, where there is potential for both short-term and longterm effects, and emphasises the importance of considering the exposure in relation to the hazard; a short period of high exposure to $2,3,7,8$-TCDD is not likely to be significant when the hazard of concern is cancer, but could be a significant issue where the hazard relates to embryofoetal exposure, because short-term high levels of maternal exposure could produce short-term high concentrations in the embryo or foetus at a critical period of development.

5.3.4.1. Non-threshold effects: For non-threshold effects, such as cancer, that have undergone risk characterisation by the use of quantitative, low-dose risk extrapolation, any increase in risk with increase in dose can be readily interpreted using the same mathematical model. Because all models approximate to a linear relationship at the low doses that would be relevant to human exposures, a twofold increase in the exposure would result in a twofold increase in calculated risk. Thus, if the intake were twice that giving a predicted lifetime risk of 1 in $10^{6}$, then the predicted lifetime risk would be 2 in $10^{6}$. Although this analysis provides the same apparent precision as the original calculation of the virtually safe dose, it is subject to the same approximations and potential errors.

5.3.4.2. Threshold effects: For threshold effects, the risk above the guidance value cannot be estimated by a simple extension of the procedure adopted to calculate the guidance value. There are two aspects to be taken into account when considering intakes above the ADI:

- the relationship between the NOAEL, or other starting point, and the true biological threshold for the critical effect that is used as the basis for setting the guidance value and

- the use of uncertainty factors to allow for possible species differences and inter-individual variability.

A continued intake above the ADI would have the effect of eroding the safety margin by the ratio of the ADI to the predicted excess intake (Renwick, 1999a). Advice given to risk managers could relate to the proportion of the population that would exceed the ADI (based on predicted intake data), the extent to which the 
ADI can be exceeded without any likely risk (for example based on the difference between the NOAEL and the likely position of the true threshold), and the duration of elevated intake considered necessary to produce an increase in risk (International Life Sciences Institute, 1999a, 1999b).

The advice should be related not only to the critical effect but also to the possibility of a risk of other effects. The latter would require a re-evaluation of the hazard database to identify effects other than the critical effect, which occurred at doses just above the NOAEL/ LOAEL. Alternative approaches to the assessment of the significance of intakes above the guidance value are provided by categorical regression analysis and probabilistic methods (see Edler et al., 2002). In categorical regression analysis toxicity data from multiple studies can be combined to give an overall picture of the doseadverse effect response data. A disadvantage of this approach, especially when combining multiple studies, is that considerable toxicological judgement is needed to rate the severity of different adverse effects reported in different toxicological investigations in order to give a uniform categorisation system. In addition, because different effects and data for different species may be combined in the same regression analysis, the interpretation of the output in terms of meaningful advice to risk managers can be very difficult. A better way to address the risks at intakes above the guidance value is the use of probabilistic methods, in which point estimates for the NOAEL and the uncertainty factor(s) are replaced by distributions (see Edler et al., 2002). Such approaches require an extensive hazard characterisation database, and even then involve assumptions that are similar in nature to simpler considerations of the doseresponse for the critical effect (see above).

Special consideration may need to be given to exceedance of the guidance value by particular groups. The guidance value for long-term exposure (e.g. ADI, TDI) is defined with respect to the dose that is likely to be without harmful effect even if continuous exposure occurs over a lifetime. Specific life-stages, such as infancy and pregnancy (see Section 5.3.1.2), that have a limited duration, are usually considered as part of hazard characterisation and the ADI/TDI/RfD would normally be calculated such that all life-stages are covered. In these circumstances, provided exposure is such that the health-based guidance value is not exceeded, even during critical life-stages, there is no cause for concern.

In this context, it is worth noting that setting an ADI on a life-stage that is particularly sensitive may result in a value that provides a very wide margin of safety for other life-stages. Therefore exceeding the guidance value only during less sensitive life-stages could be without any health implications. Equally there may be situations where exposure averaged over a lifetime does not exceed the guidance value, but does exceed the guidance value during a specific life-stage. This situation can apply in the case of exposure of infants to lipid-soluble contaminants via breast milk (e.g. 2,3,7,8-TCDD and other organohalogen compounds). In these cases advice to the risk manager requires very careful weighing of risks and benefits.

\subsubsection{Advice related to uncertainties and sensitivity analyses}

The nature of the database on which any risk characterisation is based is a key consideration. The range, relevance and quality of the individual laboratory and human studies and any information on exposure will all have a bearing on the confidence with which the risk characterisation may be applied to humans. Also these will influence the degree of uncertainty associated with any numerical risk characterisation. The narrative that accompanies the risk characterisation should explain the strengths and limitations of the hazard characterisation and intake data.

Uncertainty can be expressed numerically when intake assessment and hazard characterisation are based on mathematical calculations. Such numerical analyses can also be subject to sensitivity analyses, to test the contribution of different aspects of the database to the overall uncertainty. Sensitivity analyses can be useful in formulating advice for risk managers, and also in identifying those parts of the database that would benefit most from refinement and improvement. In most cases the risk characterisation of food chemicals involves threshold effects, the determination of a starting point for hazard characterisation, such as the NOAEL, and the application of default uncertainty factors to allow for aspects such as species differences and human variability. Neither the NOAEL, nor the default uncertainty factor is readily amenable to quantitative estimations of uncertainty, or to sensitivity analysis. The uncertainty that arises from aspects of study design, such as dose spacing, sample size and biological variability on the BMD, could be assessed as part of a sensitivity analysis. An assessment of the relative contributions from various sources of uncertainty is important in communications with the risk manager.

5.3.5.1. Uncertainties related to exposure/intake data: In epidemiology studies, the quality of the exposure assessment is contingent on the method used for quantification and the extent to which information on individual variation is obtained and utilised. Shortcomings in the quality of exposure assessment are a common source of uncertainty in epidemiology-based risk characterisation. Major uncertainties arise if exposures cannot be assessed over the time period that is relevant for the specific outcome. For example, for genotoxic compounds, any risk of cancer may be influenced by 
exposures that occurred prior to the period of intake assessment, and any change in intake prior to the induction of the tumour would influence the resulting risk characterisation. In contrast, recent intakes would normally be important for adverse pregnancy outcomes, because the critical window for risk is only a few weeks or months, assuming the compound is not persistent. However, if the compound is not excreted rapidly it may continue to pose a risk for future pregnancies, as was the case for the Yu Cheng incident in Taiwan, and the Yusho incident in Japan, where cooking oil was contaminated with PCBs (Schantz, 1996).

Many problems can be overcome if reliable biomarkers of exposure are available. A recent example is a risk assessment undertaken in the USA for $2,3,7,8$ TCDD based on a cohort study of workers in 8 chemical plants (Steenland et al., 2001). Serum 2,3,7,8-TCDD levels were measured and the workers were followed up for cancer mortality. Excess lifetime risk through age 75 was calculated using the relative risks associated with serum 2,3,7,8-TCDD levels that were found in their dose-response analysis, and applying these to background US all-cancer mortality rates. The excess risk of dying of cancer associated with a 2,3,7,8-TCDD intake of $1.0 \mathrm{pg} / \mathrm{kg}$ body weight per day was calculated to be $0.05-0.9 \%$ above a background lifetime risk of cancer death of $12.4 \%$ in the USA (Steenland et al., 2001). The results were consistent with those obtained from another cohort that had measured serum 2,3,7,8-TCDD levels (Becher et al., 1998), thereby increasing confidence in the association.

For compounds for which there is no intake prior to approval, the uncertainties related to exposure at the time of approval relate to the validity of the predicted intake estimations. For this reason, initial assumptions tend to be conservative and may considerably overestimate the actual intake occurring after the substance is approved.

\subsubsection{Uncertainties related to hazard characterisation:}

The most usual starting point for risk characterisation of threshold effects based solely on animal data is the NOAEL. The precision of the NOAEL from an animal study depends on three variables related to the quality of the study:

- the sensitivity of the toxicological endpoint and the methods used to measure it,

- the group size studied, and

- the increment between doses.

The selection of the NOAEL is usually based on a statistical consideration of the response data in the different treatment groups, although the decision may be affected by non-statistical considerations, for example the shape of the dose-response curve at higher doses and mechanistic data. The validity of the derived NOAEL in representing adequately the true no-adverseeffect level (NAEL or threshold) is a function of both the precision of the NOAEL, determined by the quality of the data, and also the steepness of the dose-response relationship. For the same size study and dose spacing, a steep dose-response curve is usually associated with narrower confidence intervals of dose estimates corresponding to prescribed effect levels than is a shallow curve, and this will affect the confidence with which the experimental NOAEL can be considered to be a reliable surrogate for the true NAEL. Although the accuracy of the NOAEL will be greater with a steeper doseresponse relationship, proximity of the NOAEL to an effect level will also be greater, so that these two will tend to balance out in relation to the confidence in the overall risk characterisation. A disadvantage of using the NOAEL as a starting point for formulating advice to risk managers is that it is not possible to quantify the degree of variability and uncertainty that may be present. All that can be said is that:

- with good toxicological methods, recommended group sizes and narrow increments between dose levels the NOAEL should be close to the true NAEL,

- NOAELs from studies utilising poor toxicological methods and/or small group sizes may be higher than the true NAEL,

- studies using wide increments between dose levels may produce NOAELs that are considerably lower than the true NAEL.

In practice, systematic characterisation of these uncertainties is not routinely undertaken.

The problems of the undefined precision in the NOAEL are reduced if a mathematical model is fitted to the dose-response data in order to derive a more robust starting point, such as the BMD. The selection of the lower $95 \%$ confidence limit on the dose giving a certain level of response, such as a 5 or $10 \%$ response allows for uncertainties associated with the dose-response data. However, to date BMDs have not been used greatly for food chemicals, and where they have been used it has not been standard practice to provide information on the associated uncertainty to risk managers as part of risk characterisation.

The starting point for risk characterisation of nonthreshold effects based solely on animal data is usually a fixed level of response, within or close to the lower end of the experimental dose range. The numbers of animals per dose group, the background rate for the tumour type under consideration, the animal survival rate at termination of the experiment and the quality of the pathological examinations will all have a bearing on the uncertainty associated with the response rate. Using a 
lower confidence limit on the level of response, rather than the actual level of response, allows for some of these uncertainties. For example, the 95\% lower confidence limit on a dose giving a $10 \%$ extra risk of tumours, adjusted for background tumour rate, in an animal study has been used as the starting point for dose-response extrapolation (Environmental Protection Agency, 1999b).

\subsection{Uncertainties related to epidemiological} data: The key characteristics for an epidemiological study to be useful for the purpose of risk characterisation are valid methods that ensure that bias is small, precision is high, and exposure is measured accurately. Because epidemiology combines aspects of human exposure and hazard characterisation, it is important to describe the extent to which unmeasured confounders could be contributing to the reported association, as well as the magnitude of errors likely in the exposure estimate.

In epidemiological research, it is common to include sensitivity analyses that evaluate the impact of various assumptions, such as model form, linearity of the doseresponse relationship, choice of cut-points for exposure categorisation, data transformation, the presence of interactions with other factors (e.g. age, gender, nutritional status, occupational exposures) on the multiplicative or additive scale, and inclusion/exclusion of extreme values.

Unknown confounders may represent an important source of uncertainty, because by definition they would not have been measured in the study, and cannot be taken into account in data analysis. Both unknown confounders, and the methods of correction for known confounders are of greatest concern when the effect size is modest, e.g. there is less than twofold increase in relative risk across the full range of exposure within the study. For strong effects (relative risk $>3$ ), unknown confounders are less likely to explain the association completely, as they would have to be strongly associated with both the exposure of interest, and with the outcome. Unknown confounders are of greater concern when the health outcome has not been well studied previously in relation to other possible causes, since prior investigations would usually have identified any strong confounding factors.

Risk characterisation is usually presented as the relative risk derived from comparing two exposure levels or different exposure conditions, for example duration. The relative risk is the ratio of the risk (or rate) of an outcome in the exposed group(s) to the risk (or rate) of the outcome in the unexposed group in a specified time period. For cohort studies, the differences in actual or absolute risk or rate, known as the risk difference or attributable risk can be estimated, as well as the relative risk. Since risk characterisation aims to provide risk managers with information about additional disease burden due to a specific exposure, it is more informative if relative risks are converted to added risk, i.e. risk difference or attributable risk. An example of this is given by the recent meta-analysis of the influence of the consumption of alcoholic beverages on breast cancer in women (Collaborative Group on Hormonal Factors in Breast Cancer, 2002). The relative risk was shown to increase by $7.1 \%$ for each additional $10 \mathrm{~g}$ per day of alcohol intake; based on this value and assuming that the association was causal, it was calculated that alcohol would be responsible for $4 \%$ of the breast cancer in developed countries.

Although epidemiological studies do not directly measure lifetime risks in the way that animal studies can, the lifetime risk can be estimated from less-than-lifetime studies in humans (Hertz-Picciotto and Holtzman, 1989; Hertz-Picciotto and $\mathrm{Hu}, 1994)$. If age-specific data on the background rates of disease are available, any measure of association obtained from epidemiological studies with less than lifetime follow-up (i.e. partial lifetime follow-up) can be used to estimate lifetime risk (van den Brandt et al., 2002), even if age-specific relative risks have not been calculated. However, significant uncertainties may arise in the risk characterisation if important life-stages are not studied in the epidemiology investigation. The risk assessment of 2,3,7,8-TCDD performed by Steenland and colleagues (2001, see above) was based on data for workers in 8 chemical plants. An important uncertainty in the risk characterisation was whether the calculated excess lifetime risk was appropriate for all life-stages including those not part of the epidemiological database. Risk characterisations based on worker cohorts would not be appropriate for neonates, infants or children, if these groups showed different susceptibilities and dose-response relationships.

5.3.5.2.2. Uncertainties related to human trial data: Investigations in humans, such as clinical trials, can provide a good basis for risk characterisation because exposure can be controlled and precisely defined, and sensitive methods of response assessment can be used. However, the applicability of such studies is limited by obvious and necessary ethical considerations. Uncertainties arise because of the normally limited numbers of subjects, their restricted age range, the short duration of administration of the food/chemical, and the shortterm follow-up for effects. Uncertainty factors are normally applied as part of hazard characterisation, if the study population is not fully representative of the exposed population.

Biomarkers of response or of effect, that have been validated in human trials, and then applied to epidemiology studies and/or animal studies can greatly reduce the uncertainties associated with hazard characterisation. 
5.3.5.2.3. Uncertainties related to animal data: Although there are uncertainties associated with the use of data from studies in animals to predict human risk, in some cases animal data will comprise the majority of hazard identification and characterisation data available (see Table 1 above). There is a wide consensus in the scientific community that data from regulatory toxicology studies in animals are valid. This consensus is based on:

- the general interspecies similarities in physiological and pathological processes, which are being substantiated by genomic analyses,

- known examples of chemicals (mainly drawn from the areas of food contaminants and human drugs) where toxicity in animals and humans is qualitatively, if not quantitatively similar, and

- the long experience that animal-based risk assessments have served to protect public health well to date.

Risk characterisation should affirm the validity of animal data in advice to both risk managers and other stakeholders including the public.

When formulating advice to risk managers, the possibility needs to be borne in mind that a hazard relevant to human health could have been missed if the usual test species were insensitive. In addition, some acute adverse effects from foods may manifest themselves in humans in ways that are not assessable or not readily monitored in animals, such as allergies or headaches. Identification of an allergenic hazard of new proteins, for example those introduced via novel foods, relies mainly on theoretical considerations by comparing its structure with those of known human allergens.

In vitro studies using animal and human tissues have the potential to provide information that can reduce uncertainty. For example, they can be used to define differences in target organ sensitivity or to provide metabolic data that can be incorporated into a PBTK model. The criteria for the use of in vitro data to define species differences in response have been the subject of an International Programme on Chemical Safety harmonisation project (2001a).

\subsection{Uncertainties related to human variability:} There may be wide interindividual variability in both the fate of substances in the body and their effects at a cellular level. In contrast to therapeutic drugs and therapeutic responses, there are very few data that define the variability within humans in response to exposure to potentially toxic chemicals, including those in foods. Increased susceptibility may arise from differences in toxicokinetic or toxicodynamic processes. Incorporation of human variability in both toxicokinetics and toxicodynamics will become increasingly important as risk assessors move towards the use of CSAFs and probabilistic approaches to risk characterisation.

Groups in the population may show a heightened susceptibility because of genetic differences, pattern of exposure, life-stage or, in the case of allergy, prior exposure to the compound. Furthermore people with chronic diseases/nutritional deficiencies and those on long-term medication may be particularly susceptible. The difference between normal human variability and susceptible groups is that the former is a continuum, whilst the latter represent subjects who show identifiable characteristics that clearly differentiate them from the rest of the population. This can influence the nature of risk characterisation; the advice to risk managers may relate to specific subgroups with greater susceptibility providing that the individuals at increased risk can recognise for themselves that they represent part of the subgroup.

Genetic diversity. It is increasingly recognised that there is a wide genetic diversity in many of the enzymes involved in the inactivation and bioactivation of foreign chemicals, with many of the pathways in humans having bimodal or trimodal distributions indicative of polymorphism. It is probable that cellular processes involved in the generation of an adverse effect will also show similar wide inter-individual variability due to genetic differences in the nature of the enzyme, the cell component, or the extent of expression of the cellular target. Initiatives, such as the SNP Consortium, are rapidly increasing the number of polymorphisms known to be present within the human genome. An important need for future risk assessment will be an understanding of the potential impact of such genetic differences on the activation, inactivation and effects of chemicals in the human body.

Knowledge on the influence of human genetic polymorphisms on toxic responses is advancing rapidly (see earlier discussion). There is increasing concern that the default uncertainty factor may not provide adequate protection in the case of certain polymorphisms. The extent to which such polymorphisms erode current safety margins is not yet clear, but should relevant toxicokinetic information be available on differences between phenotypes, either for the specific compound or for other compounds metabolised by the same polymorphic enzyme, then this information needs to be taken into account in deriving a guidance value. Advice to risk managers usually has to cover the whole population, although specific advice may be given to recognisable subgroups, for example patients with phenylketonuria are given advice related to the consumption of aspartame which is a source of phenylalanine. Population-wide screening for genetic polymorphisms is not currently conducted and so the possible influence of certain genetic polymorphisms may need to be highlighted as an additional uncertainty in the advice that is given to the risk manager. 
Intolerance including allergy. Under some circumstances the proposed guidance value(s) will not be protective of all recognisable subgroups within the population. Guidance values cannot allow for individuals who show extreme sensitivity, for example due to allergy to a food constituent. For such individuals, the only way of avoiding risk may be to avoid exposure. Under such circumstances the advice to risk managers should discuss the need to include adequate product labelling.

Life-stage. For some life-stages, e.g. the embryo and foetus, selection of the critical effect will have included consideration of sensitivity of that life-stage during hazard identification, and hence the proposed guidance value should provide adequate protection. Because of the lack of maturity of organs and metabolic systems in very young infants, the ADI is considered not to be applicable to infants under the age of 12 weeks (International Life Sciences Institute, 1992; Scientific Committee on Food, 1998). Available data indicate that neonates have reduced ability to metabolise foreign compounds, e.g. via CYP1A2 oxidation (Dorne et al., 2001a) and glucuronidation (Dorne et al., 2001b). Such variability should be taken into account in risk characterisation of chemicals that pass into breast milk or may be present in infant formulae. In contrast to neonates, most other groups of the population, including older infants and children, would be adequately covered by the default toxicokinetic uncertainty factors applied in the risk characterisation process for threshold toxicants (World Health Organization, 1999).

Current hazard identification and characterisation approaches may not adequately test for the possibly greater sensitivity of neonatal and pre-weaning infants with respect to contaminants in infant formula. There are no standard protocols for tests to assess the safety of chemicals proposed for use in, or present as a contaminant in, infant formula. In conventional reproduction studies, the newborn and pre-weaning animals can receive chemicals via maternal milk, but this will reflect maternal metabolism and does not mimic the mode of delivery to infants ingesting a chemical in infant formula directly. This uncertainty may be most relevant to effects such as neurobehavioural changes because certain aspects of brain development take place postnatally in rodents, but occur prenatally in humans. In addition, human brain development also extends over a much longer period of time postnatally as compared to rodents. Uncertainty arising from differences in the nature of the material delivered to the neonatal animal can be overcome by direct administration of the chemical by oral gavage to pre-weaning animals, but this can present practical difficulties. A number of studies of this nature have been performed to investigate developmental neurotoxicity, and the limited results available to date suggest that the current testing strategies are adequate to protect the developing foetus and infant.

\subsubsection{Advice relating to very low risks for irreversible} hazards such as cancer

Cancer is the hazard that raises the most public concern, but neither epidemiological studies nor animal studies are capable of directly measuring a level of risk sufficiently low that it could be described as negligible, such as a lifetime risk of 1 in $10^{6}$. In consequence, risk estimates for cancer are perhaps the most controversial issue in discussions on uncertainties in risk characterisation.

Few epidemiological studies are capable of identifying risk increases of the order of 1 in a 1000, much less 1 in a million. For example, it was estimated that when the background risk (cumulative probability) of developing lung cancer in United States men over age 15 is 0.06 , an occupational study of 4343 workers would be needed to detect an excess risk of 1 in a 100 and a study of 396 million workers would be needed to detect an excess risk of 1 in a million (Stayner et al., 1999). Thus, epidemiological studies have limited power to detect levels of risk that are of concern to regulators and the public.

In animal carcinogenicity studies, the number of animals per dose per sex is usually no more than 50 and a risk-specific dose corresponding to a maximum risk of 1 in a million is on average 380,000 times below the maximum tolerated dose given in the animal study (Gold et al., 1992). Thus, the actual risk at low exposures cannot be observed in the animal study and has to be derived by extrapolation which, as discussed in Section 5.2.5.3., introduces considerable uncertainty around the extrapolated value.

It is important that the advice to risk managers provides a narrative description of the nature of the evidence and the confidence intervals associated with the risk at defined human exposures or the exposures calculated to be associated with defined risks.

\subsubsection{Advice when data are sparse but indicate serious hazard}

Sometimes it may not be possible to carry out a science-based risk assessment, either because data of the required type and quality are not available, or because the current state of the science precludes the possibility of gathering the necessary data. In such circumstances, it is the task of the risk assessor to indicate clearly to the risk manager if there is the possibility of serious harmful effects on health. The risk manager then has to decide whether to adopt "the precautionary principle" and put in place appropriate risk management measures to ensure health protection.

Regarding application of the precautionary principle, the Commission of the European Communities (2000) has stated:

"The precautionary principle, which is essentially used by decision-makers in the management of risk, should not be confused with the element of caution that scientists apply in their assessment of scientific data. 
Recourse to the precautionary principle presupposes that potentially dangerous effects deriving from a phenomenon, product or process, have been identified, and that scientific evaluation does not allow the risk to be determined with sufficient certainty.

The implementation of an approach based on the precautionary principle should start with a scientific evaluation, as complete as possible, and where possible, identifying at each stage the degree of scientific uncertainty. Maintenance of the measures depends on the development of scientific knowledge in the light of which they should be re-evaluated".

It is important that the precautionary principle, which is adopted in the absence of data on intake and/or hazard, is not confused with the conservative/cautious approaches that are adopted as a normal part of risk characterisation and which have been discussed above.

\subsection{Special considerations for particular food categories}

The principles of risk characterisation for chemicals in food and diet have been extensively discussed in Sections 5.1.-5.3. In this section the applicability of these principles to the different food categories given in Section 2.4 is discussed and any special considerations highlighted.

Data from toxicological studies (in vitro and in laboratory animals) and from human studies (absorption, metabolism, excretion, tolerance trials and epidemiology) can all contribute to risk characterisation of chemicals in any food category. Nevertheless, it is recognised that the toxicological approach particularly lends itself to risk characterisation of low molecular weight chemicals and non-nutrients, while the epidemiological approach may be better suited for the risk characterisations of micronutrients, supplements, macronutrients, and whole foods.

The basis for risk characterisation is common to all food categories in that there has to be adequate characterisation of any hazard and sufficient dose-response data to establish intakes that would not result in an adverse effect, together with adequate exposure data. The main difference between the various food categories is the likelihood of developing a numerical, health-based guidance value and the methods used to convert the starting point (NOAEL, BMD, etc.) into a guidance value for adverse effects with an assumed threshold.

\subsubsection{Defined single chemical entities of low molecular weight}

This category includes low molecular weight substances deliberately added to food, such as additives and flavourings, substances present in food as residues, such as pesticides, veterinary medicines, migrants from food contact materials and processing aids, and substances present in food as inadvertent (and often unavoidable) contaminants, such as natural toxicants, inorganic and organic environmental chemicals and chemicals generated by processes such as frying. Toxicological studies play a prime role in risk characterisation of low molecular weight chemicals. A proposed decision tree on the special considerations for risk characterisation of low molecular weight chemicals is provided in Fig. 6.

\subsubsection{Hazard identification and hazard characterisa-}

tion aspects: There are well-established regulatory requirements on the range of in vitro and in vivo laboratory animal studies that should be undertaken prior to approval, together with guidelines on protocols for conducting such studies, for defined single chemical entities of low molecular weight such as additives, flavourings, substances used in food contact materials, pesticides and veterinary medicines. Such studies are designed to identify the hazards associated with all life stages. Information on human metabolism and from human trials and/or epidemiological studies may also be available, but it is often the case that only data from in vitro and animal toxicity studies will be available for defined single chemical entities of low molecular weight, particularly where pre-marketing approval is being sought. For such compounds, regulatory bodies may require gaps in the database to be filled before approval. Other low molecular weight compounds, such as natural toxicants (e.g. mycotoxins, phytoestrogens, glycoalkaloids) and organic and inorganic food contaminants (e.g. dioxins, PCBs, heavy metals) are also amenable to study by in vitro, animal and human approaches, but the availability of animal and human data varies considerably. Thus, there may be considerable uncertainties in the risk characterisation of contaminants due to gaps in the database.

It has to be acknowledged that the practical and ethical limitations on the number of animals and species that can be studied in hazard characterisation of low molecular weight chemicals contribute to uncertainty about whether all possible effects have been detected. There are also uncertainties regarding qualitative and quantitative interspecies differences which need to be acknowledged in any extrapolations that are made between animals and humans. These should be discussed in the advice to the risk manager.

5.4.1.2. Exposure assessment aspects: The methods used to make exposure assessments for low molecular weight chemicals and the matching of the exposure assessment with the hazard characterisation have been discussed earlier (see Sections 5.2.2.1. and 5.2.2.2.). The relationship between exposures used in animal toxicity studies and those encountered by humans is an important issue for low molecular weight chemicals. In animal studies the doses and duration of exposure are clearly defined and the upper end of the dose range is usually considerably 
higher than anticipated human exposure. This is done in order to enhance hazard identification, however it complicates the interpretation of the significance of animal findings for human health, particularly where they occur only at high doses. There are, for example, considerable uncertainties surrounding extrapolation from high to low doses, especially in cases where protective metabolic pathways may have been saturated. There may also be uncertainties if the route (e.g. non-oral rather than oral) or mode of administration (e.g. gavage rather than diet) in the animal studies differs from that in humans. These situations have been discussed in Section 5.2.4. These uncertainties need to be highlighted in the risk characterisation when trying to match actual or predicted human exposure to that for which hazard characterisation data are available.

Information on adverse effects in humans may be available from the literature on human poisoning cases,

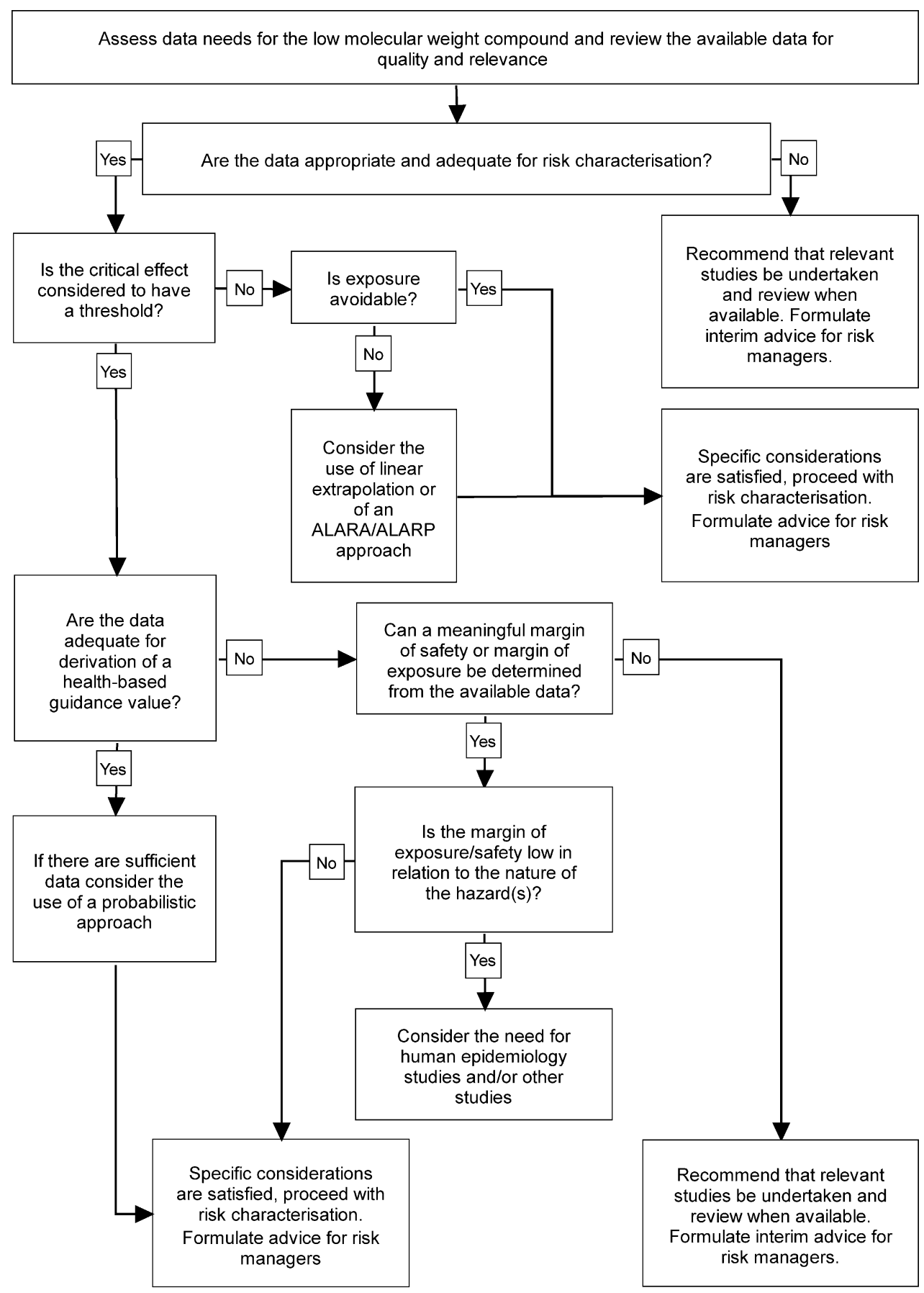

Fig. 6. Decision tree outlining the special considerations for risk characterisation of low molecular weight chemicals. (This is not intended to cover all eventualities but to indicate some matters of particular concern; it should be recognised that this is an iterative process that, depending on the availability of data in appropriate categories, may need to be revisited.) 
but the quantification of exposure may be undetermined or unreliable. In epidemiological studies, if exposure cannot be measured in individuals directly (e.g. by measuring quantitative biomarkers of exposure), reliable intake estimations can only be made if extensive data are available on the diet consumed and the actual concentrations of the low molecular weight chemical in all the relevant foods.

\subsubsection{Nature of the guidance values and other advice to} risk managers: For low molecular weight chemicals that require pre-marketing approval, the basis for the initial risk characterisation is usually a health-based guidance value with which predicted exposures can be compared. The derivation of guidance values has been common practice since the early 1960 s for the safety assessment of food additives and contaminants by the JECFA and is widely used by other regulatory and advisory bodies throughout the world, including the FAO/WHO Joint Meeting on Pesticide Residues (JMPR), the USA FDA and the EC SCF (see: International Life Sciences Institute, 2000b). As described earlier, the starting point for derivation of a health-based guidance value is usually the NOAEL. Default uncertainty factors or CSAFs are then applied to the NOAEL to derive a guidance value (see Sections 5.2.5.2. and 5.3.1.2.). The use of default uncertainty factors or CSAFs results in a deterministic estimate that does not indicate the uncertainty associated with the resulting guidance value. In most situations this is not a problem since the chemical is only approved for marketing under defined use conditions (e.g. types of food, crops, maximum levels of use, prescribed veterinary product withdrawal period, etc.) where it can be shown that the health-based guidance value is unlikely to be exceeded. However, even for chemicals requiring pre-marketing approval, their intakes after marketing may exceed the guidance value, albeit rarely on a regular basis. In such cases, the nature of the advice to risk managers about the possible health implications of exceeding the guidance value requires careful consideration and discussion (see Section 5.3.4.).

The uncertainty factors chosen for derivation of a guidance value also require discussion in the risk characterisation. Reviews of uncertainty factors (Dourson and Stara, 1983; Calabrese, 1985; Beck et al., 1993; Dourson et al., 1996; Renwick and Lazarus, 1998; Dorne et al., 2001a; Walton et al., 2001) have concluded that use of the $10 \times 10$ (100-fold) default factor to account for interspecies and inter-individual differences is appropriate in the absence of chemical-specific data, but that different situations could arise where these default factors would be either excessive or inadequate. For instance, there may be individuals with high internal exposure to a food chemical because of either enhanced absorption or reduced elimination. If this is combined with enhanced sensitivity due to either greater bioactivation or reduced cytoprotection, then those individuals might not be adequately covered by the default uncertainty factor of 10 for inter-individual differences. On the other hand, it may not be necessary to use an uncertainty factor greater than 1 for human variability when the hazard characterisation is based on a large study in humans, which includes a sufficient number of individuals from the age ranges that will be consuming the substance to cover possible differences in sensitivity. The use of additional uncertainty factors to compensate for deficiencies in the database has been discussed earlier (see Section 5.1.1.). In risk characterisation, the reasons for deviations from default values and the use of additional uncertainty factors should be fully discussed.

For low molecular weight chemicals to which there is only intermittent exposure and which do not bioaccumulate, a guidance value based on lifetime exposure may be overly conservative. If the risk characterisation shows that intermittent intakes, on the days that they occur, exceed the guidance value, then consideration must be given to the minimum duration of exposure necessary to induce the critical toxic effect on which the guidance value is based and the dose at which acute effects from exposure during one day may occur (see Section 5.3.3.1. for discussion on ARfD). It may be that further toxicological studies using different durations of dosing will be needed to provide a more secure basis for the risk characterisation.

5.4.1.4. Risk characterisation based on margin of safety: Alternative approaches used for defined single chemical entities of low molecular weight are the "margin of safety" (MOS) or "margin of exposure" (MOE) approaches. The MOS is the ratio between the NOAEL (from animal or human studies) and the actual amount of human intake/exposure. The MOE is the ratio between the NOAEL and the estimated amount of human intake/exposure. These approaches are normally used when limited toxicological data or human data exist but the hazard identification and hazard characterisation data are insufficient to set a health-based guidance value. Thus it is used most often for natural toxicants and other inadvertent food contaminants for which the hazard database may be sparse. Assessment of the adequacy of an MOS or MOE is done on a caseby-case basis. The acceptability of an MOS or MOE depends on its magnitude and is ultimately a risk management decision. To aid that decision, the nature of the toxicity involved and the uncertainties, from both the toxicological and exposure perspectives, involved in the derivation of the MOS or MOE should be clearly described in the risk characterisation. In some cases the data may even be insufficient to establish a NOAEL, but do indicate a LOAEL. Assessment of the adequacy of the margin between a LOAEL and actual or 
estimated exposures requires the same case-by-case considerations as the MOS/MOE.

In the case of some additives with good toxicity databases, but which do not show adverse effects even at the highest doses tested, the advice to risk managers may be that normal uses of such a substance (according to good manufacturing practice) would not represent a risk and that a numerical guidance value is not necessary. Such substances may be allocated an "ADI not specified" by JECFA (World Health Organization, 1987). Examples include modified starches and gums used as thickeners and stabilisers. It should be noted that "ADI not specified" is sometimes misinterpreted as meaning that an ADI had not been allocated because of toxicological concerns. An "ADI not specified" is only allocated when there are sufficient data to conclude there is no risk to be expected.

\subsubsection{Micronutrients}

Micronutrients comprise vitamins and minerals that are essential for normal growth, and physiological and biochemical functioning. The methods used for risk characterisation of low molecular weight chemicals are applicable in many respects to micronutrients, which are also defined chemical entities of low molecular weight. However there are some unique aspects, the most obvious ones being that some intake is essential for life and the margins between essential intakes and toxic intakes may be small, as is the case, for example, with vitamin A or sodium. A proposed decision tree on the special considerations for risk characterisation of micronutrients is provided in Fig. 7.

\subsubsection{Hazard identification and hazard characterisa-} tion aspects: There may be considerable prior knowledge on micronutrients, concerning chemical characterisation, absorption, distribution, excretion and metabolism, and physiological effects including benefits. However, hazard identification and hazard characterisation data are often deficient, which severely hampers the risk characterisation. Because of the importance of micronutrients for human health and nutrition, human trials and epidemiological data are much more likely to be available for micronutrients, including micronutrients administered as drugs, than for other types of low molecular weight chemical. However, such studies generally do not utilise high doses so, while they give clear indications of the doses that are nutritionally adequate or nutritionally inadequate, they provide only limited dose-response data and may provide little insight into whether higher doses cause adverse effects. This gap in essential knowledge for the risk characterisation cannot always be filled by data from laboratory animals. For many micronutrients there are only very limited hazard identification and hazard characterisation data from studies using animal models (e.g. vitamin
$\mathrm{B}_{12}$ ). In some cases it is known that some animal models are inappropriate for human risk assessment, for instance rodents do not absorb beta-carotene to the same extent as humans.

Since both deficiency and excess of a micronutrient can cause health problems, hazard characterisation requires the establishing of two separate dose-response curves. The first one is related to the intake required to satisfy minimal nutritional needs, in which the low end of the dose-response curve describes adverse effects from no intake or low intakes. Advice on this aspect is usually provided by specialists in nutrition. The second type of dose-response curve describes any adverse effects from intakes above those needed to satisfy nutritional needs. Advice on this aspect is usually provided by nutritionists and toxicologists working together.

Where animal studies are conducted for hazard identification, the generally narrow margin between the essential intake and a toxic intake has implications for dose setting. To properly explore a narrow margin, the number of doses used in a toxicity study may need to be increased and/or the range spanned by the doses may be smaller than in a conventional toxicity study for low molecular weight chemicals.

In laboratory studies attention must also be paid to possible interactions between micronutrients, especially minerals when these are given at levels in the diet that may saturate common transport and metabolic mechanisms. In some cases useful knowledge about the potential toxicity of micronutrients has been derived from field observations following poisoning or oversupplementation of farm animals (e.g. with molybdenum or selenium).

A further unique aspect of hazard identification and hazard characterisation of micronutrients is that not only does the potential toxicity of the nutrient itself need to be considered but also, for some micronutrients used for food fortification or in dietary supplements, the potential toxicity of the chemical form in which it is consumed should be considered. Micronutrients may be added or used as such, or as derivatives such as salts, enriched yeasts, pro-vitamins, acetylated amino acids, etc. Not only does the inherent toxicity of the chemical form of a micronutrient need to be considered, but also the bioavailability of the nutrient from the chemical form in which it is consumed. Either low or high bioavailability of a micronutrient from its source (compared with its bioavailability when naturally present in food) may affect the risk characterisation.

The mode of intake may also trigger the requirement for a particular type of risk characterisation. For example, risk characterisation of dietary supplements, taken as bolus doses in the form of tablets or capsules, may need to consider acute toxicity (see Section 5.3.3.1. for discussion of ARfDs). 
5.4.2.2. Exposure assessment aspects: Given the often small margin between essential intakes and toxic intakes of micronutrients, it is particularly important that intakes from all sources are taken into account. Micronutrients are found in foods, fortified foods, dietary supplements and drugs. Exposure information on micronutrients in whole foods is readily available from food composition tables that are traditionally focused on micro- and macronutrients. Information on levels of micronutrients in processed foods, the doses available in the form of dietary supplements (and who is consuming them) may be less readily available. Biomarkers of exposure, such as blood concentrations of nutrients may be valuable in determining total intakes.

5.4.2.3. Nature of the guidance values and other advice to risk managers: Since both deficiency and excess of a micronutrient can cause health problems, two guidance values for a micronutrient may be expressed. One type of guidance value refers to the recommended daily

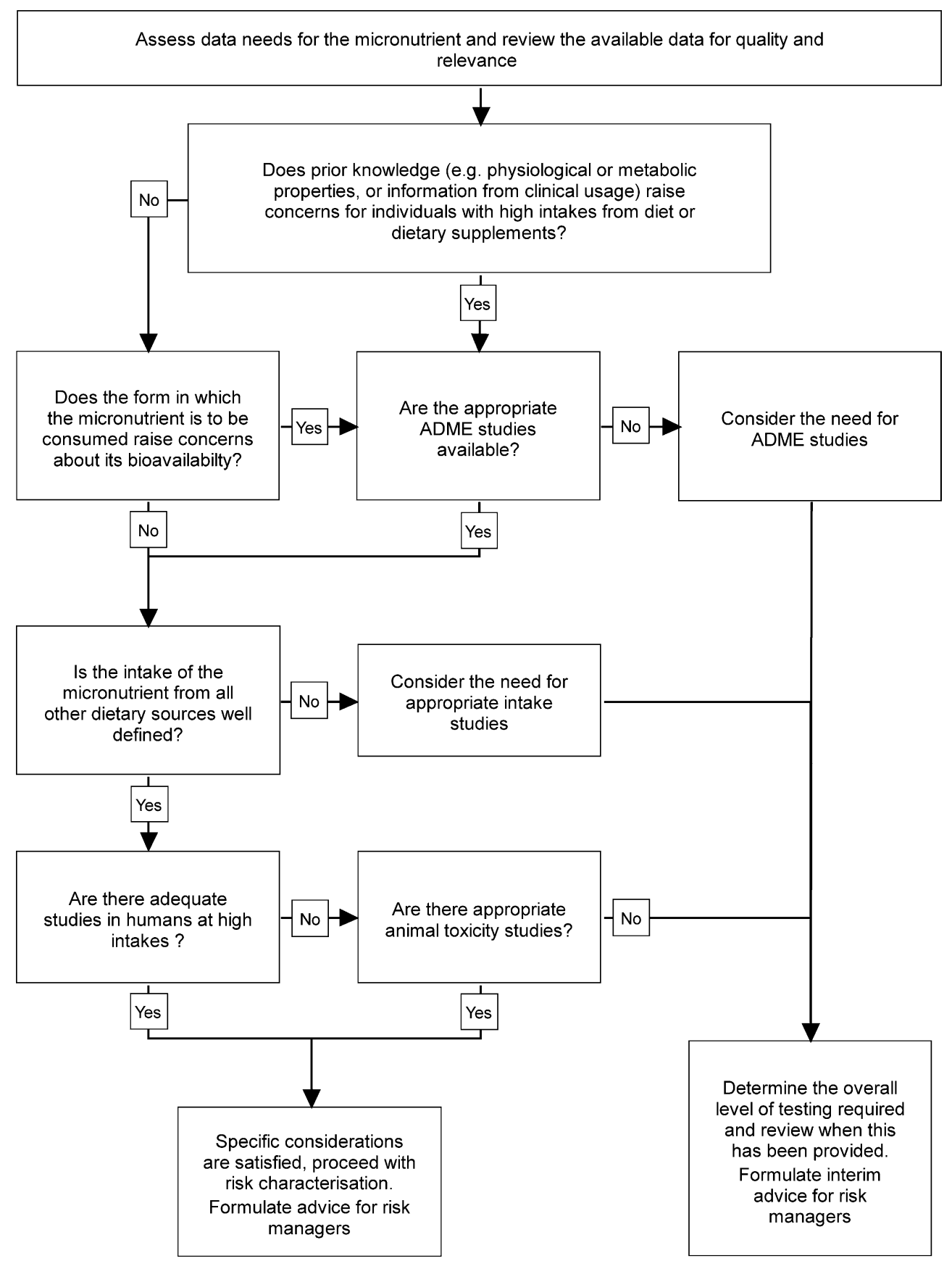

Fig. 7. Decision tree outlining the special considerations for risk characterisation of micronutrients. The parenthetical comments in the legend to Fig. 6 also apply here. 
amount below which there is risk of deficiency or suboptimal functioning, such as the Population Reference Intakes (PRIs) for micronutrients elaborated by the EC's Scientific Committee for Food (1993), or the Dietary Reference Intakes (DRIs) elaborated by the USA and Canada (Institute of Medicine, 1997). The other type of guidance value is a UL, which is defined as the maximum level of daily chronic intake of a nutrient from all sources judged to be unlikely to pose a risk of adverse health effects to humans (Scientific Committee on Food, 2000c). The setting of a UL includes consideration of what is physiologically tolerable as well as consideration of the probability of an adverse effect occurring at some specified level of exposure.

If UL guidance values for micronutrients are based on animal studies, it is often not possible to use the usual default uncertainty factor of 100 that is applied to experimental NOAELs in the case of low molecular weight chemicals, despite the fact that the same uncertainties are inherent in the use of such data for risk characterisation. While it may be possible to administer relatively high doses to animals for the purposes of hazard identification and characterisation, use of the usual default uncertainty factors could result in a UL below the recommended daily amount for nutritional needs. For this reason, margins of safety of between 1 and 10 are often considered appropriate; the size of the factor chosen depends on the quality of the database and whether the NOAEL is from human or animal studies. Interpretation of the adequacy of the margin of safety requires expert judgement on a case-by-case basis, taking into account the nature of the material, the potential hazard, if any, at high doses, and the highest potential exposure. In using low uncertainty factors, it is implicit that the risk characterisation may underestimate the true risk and this should be pointed out in the advice to risk managers. When margins of safety are small, the advice to risk managers should include a discussion of the nature of the adverse effect(s) and the dose-response, together with any uncertainties in the exposure estimates.

It should also be noted that both the lower and upper guidance values for micronutrients may differ for different human subpopulations, such as infants, children, pregnant women and the elderly. This is because of their differing physiological states and therefore differing nutritional requirements and susceptibilities to adverse effects at different life stages. Thus, the risk characterisation may need to consider a number of PRI/DRI and UL values for various population subgroups and ensure that the exposure estimate for the relevant subgroup is integrated with the appropriate guidance values.

A challenge for the interpretation of observational epidemiology studies is that the high correlation among micronutrients in the human diet makes it difficult to distinguish effects of specific vitamins or minerals. This problem arises because some nutrients cluster within foods or food groups. A large epidemiological study may be able to disentangle these factors, if the correlation between nutrients is not too high. However, statistical collinearity may represent an intractable problem. This problem has sometimes been addressed by examining food groups, rather than micronutrients; but this alters the question addressed, and does not provide risk characterisation for an individual nutrient.

\subsubsection{Macronutrients}

Macronutrients include dietary lipids, proteins and carbohydrates, their sub-components and macronutrient substitutes. The term 'macronutrients' does not refer to molecular size but derives from the fact that they are present in the food/diet in substantial quantities. They may be currently available macronutrients or new types of macronutrient (see Section 5.4.4. below for comment on scope with respect to novel foods). A proposed decision tree on the special considerations for risk characterisation of macronutrients is provided in Fig. 8 .

5.4.3.1. Hazard identification and hazard characterisation aspects: In the hazard characterisation of macronutrients consideration needs to be given to tolerance, toxicological potential and nutritional impact. As with micronutrients, there may be considerable prior knowledge about macronutrients, particularly concerning chemical characterisation, absorption, distribution, excretion and metabolism and nutritional effects. Human studies play a prime role in risk characterisation of macronutrients. Local effects on the gut, such as changes in the microflora, and on the absorption of other nutrients may be important for hazard characterisation.

A primary consideration is whether the macronutrient can be shown to be equivalent to a traditional food counterpart. If this is the case, prior knowledge and chemical characterisation may obviate the need for animal or human hazard characterisation studies. For example, a new type of fat replacer comprising microparticulated egg and milk proteins was approved for use by the UK Advisory Committee on Novel Foods and Processes (ACNFP), based on existing knowledge about the raw materials and the production process (Advisory Committee on Novel Foods and Processes, 1989). Even in cases where there is a close traditional counterpart, consideration may need to be given to the presence of new toxicants or changed levels of existing toxicants.

Hazard identification and hazard characterisation using animal studies may not be possible because addition of bulk macroingredients to experimental diets, in amounts that are exaggerated relative to the human diet, may render them unpalatable and/or cause nutritional imbalance. Under these circumstances, attribution of 
cause to any adverse effect observed becomes difficult, as does interpretation of its significance for human health. The issue of the utility of animal studies for macroingredients has been considered in some detail in an earlier FOSIE publication (Dybing et al., 2002). Tests for low molecular weight chemicals deliberately use high doses to compensate for a limited number of test animals: the dilemma for macronutrients could perhaps be partly mitigated by the use of larger numbers of animals to detect low incidence effects, but this would have animal welfare implications and may give only a small information gain at a high cost. In the absence of appropriate human data, a better approach might be the selection of an animal model that had similar gastrointestinal physiology to humans (e.g. the minipig), thereby reducing the uncertainties of interspecies extrapolation.

Because of these and other considerations, the role of human trials and observational studies are widely viewed as particularly important for macronutrients. However, it should be stressed that the utility of human trials is not primarily for establishing safety but for investigating aspects such as absorption, metabolism and excretion, nutritional issues and tolerance. Human trials should be conducted only when other considerations have given sufficient reassurance that untoward reactions are unlikely. In both animal and human studies on bulk components, careful consideration needs to be given to ways of obtaining as much information as possible, what endpoints and biomarkers (see Section 5.2.4.)

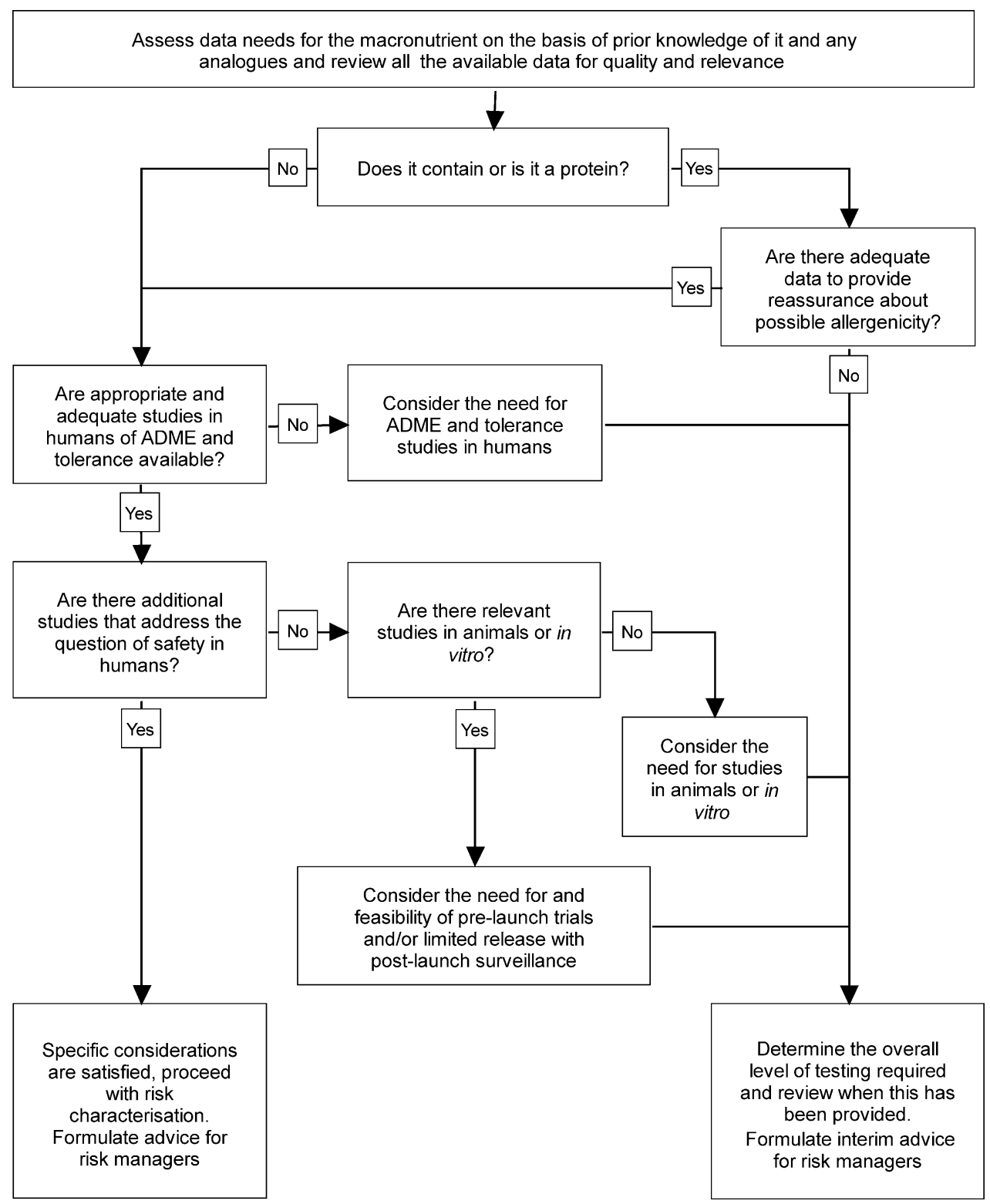

Fig. 8. Decision tree outlining the special considerations for risk characterisation of macronutrients. The parenthetical comments in the legend to Fig. 6 also apply here. 
might be used and how any measurements are to be interpreted in the risk characterisation. Apart from differences in metabolism by the gut flora, major species differences in the handling of macroingredients (biokinetics) are not expected, because they would be metabolised by the constitutive enzymes of intermediary metabolism and not by enzymes which can show wide interspecies differences, such as the cytochrome P450s. However, the suitability of the animal model needs to be considered; for example, rats do not handle fat in the same way as humans. Thus, the possibility of interspecies differences and human variability in the relevant metabolising enzymes need to be examined in each case in order to predict any likely exceptions.

Adverse effects in humans may be identified from the results of epidemiological studies. Human trials can also be used, in conjunction with prior knowledge on possible adverse effects, to look at relevant health endpoints in order to provide confirmation of the absence of adverse effects. For example, biomarkers of risk factors for coronary heart disease have been followed when fats are administered, or changes in serum beta-carotene levels with sustained consumption of phytosterol-enriched foods (Scientific Committee on Food, 2002a). In vitro or in silico studies can provide information on digestibility of macronutrients and assess the likely impact on gut microflora. In the case of proteins, particularly those resistant to pepsin digestion, ultimately only human data will establish any potential to cause allergy. However, in the case of new types of proteins, a theoretical consideration of sequence homologies and/ or structural similarities to known allergens can be made, which may provide an alert about the possibility of allergic reactions.

5.4.3.2. Exposure assessment aspects: As with micronutrients, exposure information on macronutrients in existing whole foods is readily available from food composition tables. Information on levels of macronutrients in processed foods is also frequently available from nutritional labelling. In the case of a new type of macronutrient, exposure will need to be estimated from proposed uses, in which case, the exposure considerations for future use/exposure discussed earlier will apply, see Section 5.2.2.2 and Table 1. Accurate prediction of the likely commercial success, and therefore intakes, of a new type of macroingredient is particularly difficult and post-launch monitoring can play an important role in checking whether the risk characterisation was appropriate to actual exposure. New types of macroingredients may also be incorporated into a wider variety of foods after approval than those originally anticipated. In such cases aggregate risks may need to be assessed (see Section 5.3.2.3.) as has been done, for example, for multiple dietary sources of phytosterol esters (Scientific Committee on Food, 2002a).
5.4.3.3. Nature of the guidance values and other advice to risk managers: Macronutrients are similar to micronutrients in that there may need to be more than one guidance value. One would be based on the minimal requirements to meet nutritional needs, for example, guidance values on protein, lipid and carbohydrate contents of infant formula to meet growing infants' nutritional requirements (Scientific Committee for Food, 1984). The other type of guidance value would be analogous to a UL and based on knowledge of any adverse effects at high intakes, such as a recommended upper limit for the daily intake of saturated fats in relation to the risk of cardiovascular disease, or an upper limit for the daily intake of alcohol in relation to the risk of breast or other cancers.

In some cases, a guidance value is not established, for example when the substance is a close analogue of a natural macronutrient and/or appropriately performed studies have not identified an adverse effect. Under such circumstances, a margin of safety approach may be preferable, since it is not possible in animal studies to exaggerate the dosage sufficiently to accommodate the usual uncertainty factors. Comparison of the potential human intake with experimental data showing no adverse effects will give the margin of safety. For essentially "non-toxic" macronutrients the experimental data may not show any adverse effects at the doses tested, in which case the calculated margin of safety can be regarded as the minimum likely value. As discussed earlier, see Section 5.4.2.3., interpretation of the adequacy of the margin of safety requires expert judgement on a case-by-case basis. Alternatively, the advice to risk managers may be that normal uses of the macronutrient would not represent a risk and that a numerical guidance value is not necessary.

\subsubsection{Whole foods}

Whole foods may be defined as single foods, which are usually complex mixtures that may or may not be modified by processing. They may form a high proportion of the diet for some consumers, with possible undesirable nutritional impacts, depending on amounts consumed. Their metabolism is complex and some interactions with other dietary components can be expected. They may be foods currently on the market or new types of foods not yet on the market for which approval is being sought under novel foods legislation. A proposed decision tree for assessing the special data needs for risk characterisation of whole foods is provided in Fig. 9.

5.4.4.1. Hazard identification and hazard characterisation aspects: Whole foods, like macronutrients, need consideration of the matter of tolerance, toxicological potential and nutritional impact during the hazard characterisation. As part of problem formulation, it 
should be considered whether assessment of the whole food could be done by breaking it down into its individual components, since there is likely to be prior knowledge on many of the components. Hazard characterisation can then proceed by exclusion of traditionally safe components from further consideration (unless present in higher amounts than in traditional foods) and focusing on the remaining component(s). If the remaining components fall into other food categories, i.e. low molecular weight chemicals, micronutrients, or macronutrients, and are amenable to separate evaluation, then the hazard characterisation should be appropriate to the relevant food category. A fractional approach to testing may be possible, with the advantage of better identifying any hazardous components, but there are as yet no formal guidelines for such an approach. Even in cases

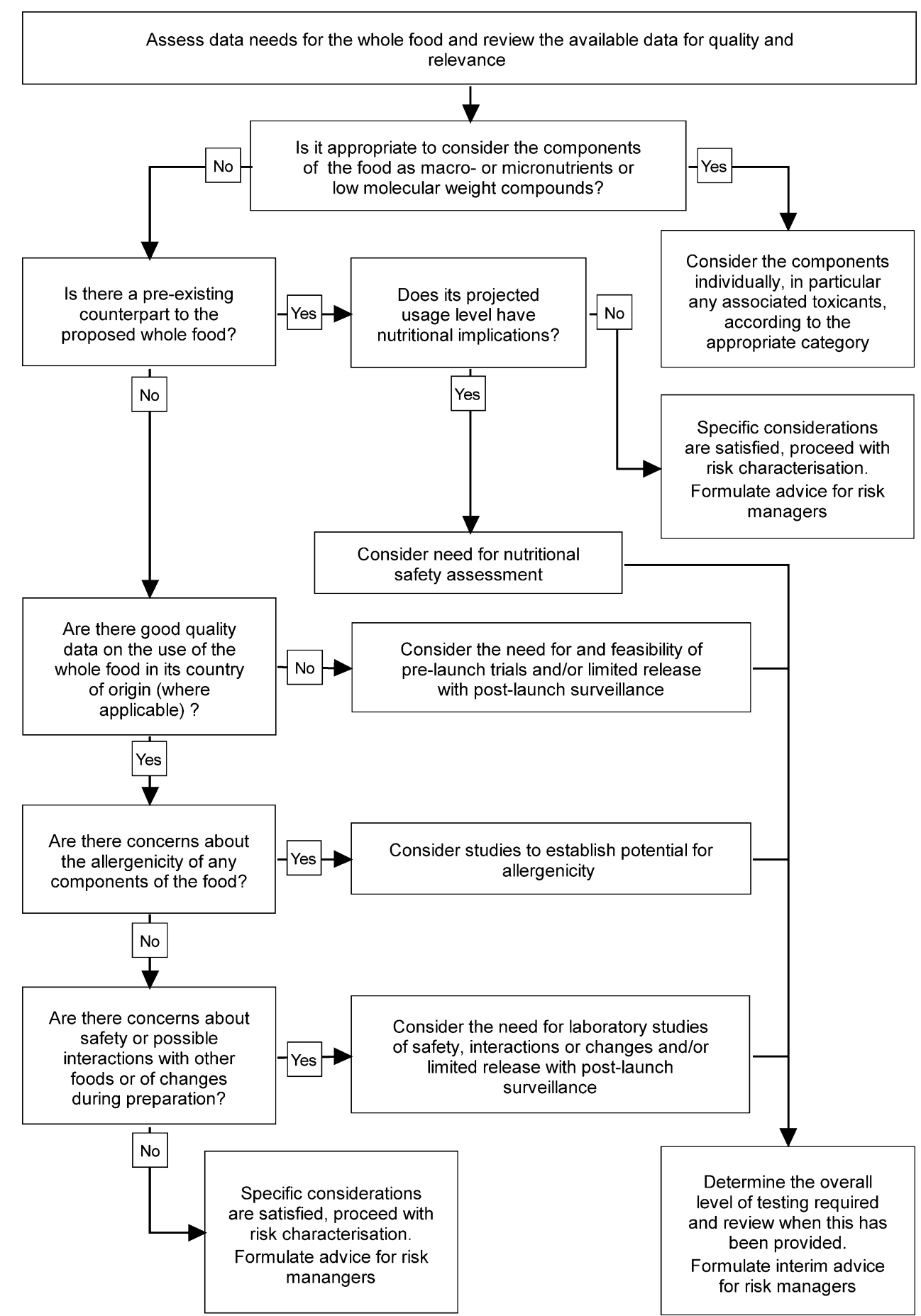

Fig. 9. Decision tree outlining the special considerations for risk characterisation of whole foods. The parenthetical comments in the legend to Fig. 6 also apply here. 
where there is a close traditional counterpart, consideration may need to be given to the presence of new toxicants or changed levels of existing toxicants.

Part of the hazard characterisation may need to focus on the potential for interactions of substances within the food. One such example would be the potential of the amino acid asparagine in potatoes to interact with other naturally-occurring components to form acrylamide when the potato is cooked at high temperatures such as in frying (Mottram et al., 2002). The hazard characterisation may also need to consider interactions between the whole food and other dietary components. For example, whole foods may interact with mineral micronutrients in other foods, altering the bioavailability of the mineral, as in the case of fibrous foods reducing mineral bioavailability, or orange juice enhancing the absorption of iron. Hazard identification and characterisation may also need to consider the presence of natural toxicants.

Methods for hazard characterisation of new types of foods have been discussed in national and international fora (Advisory Committee on Novel Foods and Processes, 1991, 1992; Health Canada, 1994; Scientific Committee for Food, 1997; Life Sciences Research Office, 1999), but as yet there is no world-wide consensus on the most appropriate approaches, other than to recommend that a case-by-case consideration is needed. The initial approach for new types of foods requires consideration of the extent to which the novel food differs from any traditional counterpart, or other related products, and hence whether it can be considered as safe as traditional counterparts/related products. Where a food is deemed novel because it has not hitherto been consumed in EU countries, there may be relevant hazard characterisation data from other areas of the world where the food has been consumed.

As for macronutrients, hazard identification and hazard characterisation using animal studies may not be possible because addition of bulk foods to experimental diets of rodents, in amounts that are exaggerated relative to the human diet, may cause problems. Other animal models with gastrointestinal physiology closer to that of humans may need to be considered.

Human trials on whole foods, including novel foods, will only be performed when no serious adverse effects are expected. Trials may be blinded or unblinded, involve cross-over, in which the effects of the food are compared with a conventional counterpart in the same individuals, or randomised controlled trials. Advantages of randomised controlled trials are that the exposure can be well characterised and the substance can be administered in a blinded way, in order to remove participant and observer bias. Additionally, random allocation to exposure groups reduces the probability of confounding, particularly in large trials. Such studies may be particularly suitable for the pre-launch assessment of novel foods. Post-launch monitoring can be used to survey the occurrence of unexpected hazards associated with new products or ingredients.

As for macronutrients, observational epidemiological data may also be available, identifying adverse effects, including allergic reactions. For novel foods that contain proteins, in vitro digestibility and sequence homology and/or structural similarity to known allergens may provide clues about possible adverse effects.

5.4.4.2. Exposure assessment aspects: Exposure information on whole foods currently on the market will be available from dietary surveys. Where the food is novel, exposure will need to be estimated from proposed uses, in which case, the exposure considerations for future use/exposure discussed earlier will apply, see Section 5.2.2.2 and Table 1. As for novel macronutrients, accurate prediction of the likely commercial success, and therefore intakes, of a novel food is particularly difficult and here post-launch monitoring can play an important role in checking whether the risk characterisation was appropriate to the exposure.

5.4.4.3. Nature of the guidance values and other advice to risk managers: Advice on whole foods does not usually involve guidance values, but instead takes the form of recommendations, which may include a quantitative element. For example, advice may be to increase or decrease daily intakes (e.g. to decrease the consumption of red meat). For novel foods, the advice to risk managers is usually whether to approve its use or not, but may also include advice about recommended maximum daily intakes.

The advice to risk managers may need to include an explanation of why higher margins of safety are used for low molecular weight chemicals, whereas for whole foods and novel foods margins are lower between the amounts normally/likely to be consumed and the amounts which may have been tested and found to be without effects in animal or human trials. Acceptance or not of these lower margins of safety is a risk management decision, but historically acceptance of lower margins of safety has been common for whole foods (as well as for nutrients and micronutrients). Both whole foods and novel foods may also have known or claimed benefits and these will need to be taken into account by risk managers in reaching a decision on the balance of risks and benefits.

\subsubsection{Food processing}

The effects of processing on foods require a separate evaluation from the safety assessment of the unprocessed food itself. New food processes, such as ohmic heating for food sterilisation, in which an alternating current is passed through foods resulting in their heating up due to their resistance (Advisory Committee on Novel Foods and Processes, 1992), or established food 
processes, such as the influence of cooking practices on polycyclic aromatic hydrocarbon formation (Scientific Committee on Food, 2002b) may require risk characterisation. The risk characterisation is strongly weighted towards characterisation of the processed material itself, with a view to identifying any changes in components as a result of the processing, assessing the potential variability of the process and any resulting variability in product characterisation. Novel processes should be compared with any comparable existing processes to establish the nature of any differences and whether these may need further evaluation.

The advice to risk managers in such cases needs to focus on any nutritional, toxicological or microbiological consequences of the process for food safety, rather than on the safety of the process itself. It will need to address the extent to which the foods processed remain the same and the extent to which the process results in any constituents that are usually present disappearing, while other chemicals not normally present may be newly generated. Some of the constituents that disappear may be important micronutrients, such as heat-labile vitamins. Some processes may induce the formation of undesirable substances, for example acrylamide from frying, grilling and baking of carbohydrate-containing foods (Swedish National Food Authority, 2002).

\section{Gaps and research needs}

In the previous sections various areas of the risk characterisation process have been identified as requiring either further developments of methodology or improvements in background scientific knowledge in order to strengthen the outcome of the overall process. In this section the key research needs identified are summarised. Some research needs have been included that are not directly related to the risk characterisation process, however, if these were to be satisfied, they would provide a more secure foundation for the process. These research needs are grouped into categories covering:

1. Structured approaches to risk assessment,

2. Methodology of hazard identification,

3. Methodology of intake estimation,

4. Assessment of critical endpoints,

5. Increased use of mechanistic knowledge

6. Use of critical endpoints in determining guidance values for food chemicals,

7. Use of probabilistic approaches and categorical regression,

8. Risk/benefit.

The research needs within each category are prioritised with the higher priority items at the head of the list. The research will require a multidisciplinary approach involving the co-operation of, for example, toxicologists, epidemiologists, mathematicians, model developers and risk assessors.

\subsection{Structured approaches to risk assessment}

The introduction of new procedures into the risk characterisation process will increase its complexity and, in order to improve its transparency, will require that it should be reported in a manner that will be easily comprehended by individuals not directly involved in the process. Various steps could be taken to reduce the uncertainties in the risk assessment process and to increase the transparency:

1. Improvement of the scientific basis for quantitative risk assessment methodologies for thresholded and non-thresholded toxicity to allow the prediction of risks and thus enable risk managers to decide better what measures are appropriate.

2. Replacement of general default uncertainty factors, e.g. 10 for different species and 10 for human variability, by the development and validation of appropriate uncertainty factors that are related to the metabolic fate of the compound and its mechanism/mode of action.

3. Development of a concept to combine aspects of uncertainty (safety) factors and nutritional homeostasis, in particular for micronutrients, macronutrients, novel foods and whole foods.

4. Development of methodologies on Good Evaluation Practice.

5. Development of a narrative framework for describing critical conclusions on, e.g. mechanism, absence of threshold, and species specificity of effect.

\subsection{Methodology of hazard identification}

There is a need for the development and validation of:

1. Better interspecies extrapolations by close collaboration of nutritionists, toxicologists, epidemiologists and mathematicians, particularly in areas such as peri- and postnatal development (see also Section 6.1., items 2 and 3).

2. Improved methods for the detection of delayed onset toxicity and, in particular, better models for human neuropsychological syndromes.

3. Animal models for the identification of acute adverse reactions to foods and food chemicals, which are classifiable as intolerance or allergy.

4. Animal models for common disease states in humans to allow targeted evaluation of the effect of such states on the toxicity of foods and food chemicals when necessary. 


\subsection{Methodology of intake estimation}

There are requirements for the development of:

1. Improved harmonisation of European food consumption survey methods in order to provide better assessments of exposure to food chemicals, with particular emphasis on the determination of reasonable values for extreme exposure and potential under-reporting.

2. Improved procedures for the assessment of exposure arising from intake by different routes, or by the intake of different chemicals with similar modes of action.

3. A harmonised pan-European food composition database comprising all food categories and specifying appropriate sampling and analytical methods.

4. Models for the study of the effects of variation of the food matrix on the absorption of individual chemical components of that food.

5. A better understanding of the effects of processing on food constituents in so far as it affects exposure assessment.

6. Models for exposure to allergens and methods to predict the probability of adverse effects arising from such exposures (e.g. by including the incorporation of variables representing individual susceptibility in probabilistic models).

7. Procedures to provide a link between models of exposure that evaluate the amount of a chemical entering the digestive tract and those models that estimate internal exposure.

\subsection{Assessment of critical endpoints}

There is a need for refinements related to critical endpoints, from either human or animal studies (possibly supported by retrospective analysis of existing data) to provide or improve:

1. Identification and development of reliable and sensitive molecular or clinical biomarkers of toxicologically-relevant endpoints (including those in the fields of genomics and proteomics). These biomarkers should be effective in shortterm studies, predictive of the risk of disease, and capable of being used in epidemiological studies on a relatively large scale at low cost.

2. Knowledge of the shape of the dose-response curve at low doses of genotoxic and non-genotoxic carcinogens (see also Section 6.1., item 1).

3. Further development of a parallelogram approach based on the comparison of in vitro assays utilising animal and human cells, and of biomarker-based analysis of both animal and human exposure data.
4. Development of an accepted framework for the classification of effects as either adverse or adaptive.

5. Optimisation of study design with respect to the numbers of animals and doses used.

\subsection{Increased use of mechanistic knowledge}

For extrapolation of the consequences of adverse effects from observations in animals to predictions in humans it is necessary to have a good understanding of the differences between species of the manner in which adverse effects may be induced; some key points are the need for:

1. Improved understanding of the biological basis for the extrapolation of different endpoints to humans by including toxicokinetics and target dose estimation in a combined toxicokinetic/ toxicodynamic model.

2. Increased insights into the processes that lead to certain toxic endpoints, such as those related to reproduction, the immune system and the central nervous system, in order to assess an endpoint's reliability and relevance in human risk assessment.

3. Improved understanding of the biological basis for combination effects of two or more compounds.

\subsection{Use of critical endpoints in determining guidance values for food chemicals}

Having determined critical endpoints and been satisfied that there is no mechanistic evidence that would exclude an endpoint from occurring in humans, the group undertaking the risk assessment would proceed to the establishment of guidance values for the food component involved. At this stage various aspects would benefit from further investigation:

1. Identification and application of practical "thresholds" for genotoxic compounds, based on data from epidemiological studies of unavoidable contaminants.

2. Development of a structured approach to the application of data on known polymorphisms in humans and animals in the risk assessment process.

3. Role of genetic inheritance and its interaction with other factors (e.g. nutritional health, microbial disease status, gut flora, age, gender, physiological status) in accounting for variation and the values of relevant uncertainty factors to be applied.

4. Reassessment of the validity of the default uncertainty factors by analysis of historical data on differences between humans and experimental animals or within human subpopulations. 
5. Identifying circumstances for which it would be appropriate to derive acute reference doses for compounds other than pesticides.

\subsection{Use of probabilistic approaches and categorical regression}

Alternative probabilistic approaches within the risk assessment process may allow better use of the available data, and could provide a basis for the validation of the overall process:

1. Introduction and validation of probabilistic approaches to exposure assessment, estimation of the dose-response curve, toxicokinetic and toxicodynamic parameters, measures of human variation and the precision of the data would provide a more rational and realistic framework for risk characterisation and facilitate the integration of toxicology and epidemiology.

2. Evaluation of the contribution of techniques of sparse data analysis in study design and interpretation for hazard characterisation and exposure assessment.

3. Development of criteria for the assignment of categories of severity of effect (magnitude of effect, not nature of effect) and for combining studies (e.g. by weighting small and large scale studies) in order to apply categorical regression.

\subsection{Risk/benefit}

The FOSIE project addressed the overall process of risk assessment but did not address the issue of riskbenefit analysis. It became clear during the course of the FOSIE discussions that there is a need to consider the development and validation of methods to combine assessment of risk and benefits:

1. Development and validation of methods to combine health risks and health benefits. Since exposures can be protective for particular disease(s) whilst enhancing the risk of other diseases, the further development and use of composite public health measures will be valuable in the estimation of optimal ranges of exposures.

\section{Conclusions}

Risk characterisation is the process that brings together the available data on hazard characterisation and exposure assessment, the nature, quantity, and quality of which will vary from substance to substance. In consequence it is not possible, or even desirable, to provide detailed guidelines or a check list for how to perform risk characterisation. This paper has therefore discussed the key features of risk characterisation for food chemicals; whilst the focus has inevitably been on low molecular weight chemicals, about which there is most knowledge, the applicability of the paradigm to other food categories has been discussed.

Risk characterisation is an iterative process to which all stakeholders should contribute, as appropriate, and which may evolve as the hazard and exposure data are collected and assessed. Central to risk characterisation is the scientific evaluation of data on hazard identification and characterisation, and on exposure. It is important that the scientific evaluation is independent and not influenced by outside interests, such as industrial sponsors or pressure groups, or by risk management considerations. However it is also important that the output of the process is usable by risk managers, and is in a form that can be communicated to, and understood by, all stakeholders.

The output of risk characterisation may take various forms including:

- Advice that current intakes or patterns of use do not represent an appreciable risk to human health,

- Derivation of a health-based guidance value, such as an ADI, to indicate a level of intake that could be consumed daily over long periods without appreciable risk to human health,

- A quantitative estimate of the risks associated with different levels of intake,

- Advice on the ratio between doses known not to produce adverse effects in experimental systems and the estimated human intake, i.e. a margin of safety, and

- Advice that, because of the nature of the hazard, an intake that would be without appreciable risk to human health cannot be determined.

For low molecular weight compounds, the extents of hazard and exposure data considered to be necessary for adequate risk characterisation are related to the predicted exposure. For substances such as flavours and packaging migrants, for which human intakes are extremely low, it may be possible to undertake risk characterisation and formulate advice in the absence of chemical-specific toxicity data. In contrast, an extensive hazard identification and characterisation database would be necessary for compounds that may be present in significant amounts in food due to intentional uses in the food chain or from direct addition to food. Risk characterisation for contaminants may be required in situations when the available data may not be adequate for a comprehensive evaluation, and under these circumstances additional areas of uncertainty would need to be taken into account. 
The advice to risk managers should describe clearly the nature of the available hazard identification and characterisation data, and the way that the data have been interpreted to derive the advice given to risk managers. There are always uncertainties in the interpretation of toxicity data, such as extrapolation of data from high dose to lower intake levels, inter-species extrapolation, and consideration of human variability and subgroups of the population. The method(s) by which uncertainties have been taken into account, for example by the use of default uncertainty factors, should be described as part of the advice to risk managers.

There are also uncertainties in intake estimations, such as prediction of the extent of use for a substance prior to its approval, or the consistency of individual patterns of food intake over time; for example, average intakes from periods as short as one week are used as estimates of average long-term intake. Again the nature and extent of any uncertainty and imprecision in the intake part of the risk characterisation should be identified in the advice to risk managers.

In practice any uncertainties inherent in risk characterisation are taken into account by the use of conservative assumptions and approaches. As more information becomes available on a substance, then uncertainties are reduced and default approaches can be replaced by chemical-specific information. However, the generation of chemical-specific information cannot be produced without costs in terms of the need for more studies in humans and/or animals. Therefore some degree of uncertainty will remain a constitutive part of risk characterisation. Refinements to default approaches and assumptions will provide a more secure basis to risk characterisation. A number of areas of generic research have been identified in this report that could improve the process of risk characterisation without the need for an increase in chemical-specific information.

\section{Acknowledgements}

ILSI Europe would like to thank all of the contributors to the FOSIE concerted action. For the final phase of the project, we would like to thank all of the Individual Theme Group (ITG) G members for preparing the paper, workshop participants of the Third Plenary Meeting for having reviewed the paper prior to its publication and especially Prof. Andrew Renwick, Dr. Sue Barlow and Prof. Irva Hertz-Picciotto for finalising the paper. We are grateful to Prof. Robert Kroes for having acted as chair for the ITG G. Overall, we would like to thank the ILSI Europe Risk Assessment of Chemicals in Food Task Force members and FOSIE steering committee members for their support and guidance. Finally, we would like to thank the European Commission for the financial support. As co-ordinator of the Concerted Action, ILSI Europe would like to express their profound gratefulness to all of them.
Co-ordinator:
Dr. Berry Danse ${ }^{\dagger}$
Scientific Advisor:
Project Manager:
Dr. Juliane Kleiner
Sandra Tuijtelaars, Msc
ILSI Europe, 83 Avenue E. Mounier, Box 6, B-1200 Brussels, Belgium

Annex 1. List of participants of the Third FOSIE Plenary Meeting, 23-25 October 2002, Lisbon, Portugal

Dr. P. Abbott

Dr. S. Barlow

Dr. R. Battaglia

Dr. S. Bausch

Dr. D. Benford

Dr. A. Boenke

Prof. A. Boobis

Prof. J. Bridges

Prof. B. Brunekreef

Prof. E. Dybing

Dr. L. Edler

Prof. G. Eisenbrand

Dr. P. Fenner-Crisp

Prof. C.L. Galli

Prof. M. Gibney

Prof. J. Gilbert

Dr. J. Godfrey

Dr. J. Greig

Ir. C. Guittard

Prof. I. Hertz-Picciotto

Dr. N. Ito
Food Standards Australia New Zealand (FSANZ)

MRC Institute for Environment and Health

Swiss Quality Testing Services (SQTS)

TNO Nutrition and Food Research Institute

Food Standards Agency

European Commission- DG Research

Imperial College

University of Surrey

IRAS - University of Utrecht

Norwegian Institute of Public Health (NIPH)

German Cancer Research Centre

University of Kaiserslautern

ILSI Risk Science Institute (RSI)

University of Milan

Institute of European Food Studies (IEFS)

DEFRA - Central Science Laboratory

European Research into Consumer Affairs (ERICA)

NutraSweet

University of California, Davis

University of Nagoya City
AUS

UK

$\mathrm{CH}$

$\mathrm{NL}$

UK

B

UK

UK

NL

N

D

D

USA

I

IRL

UK

UK

UK

F

USA

J 
Dr. A. Kadry

Prof. F. Kemper

Ms. B. Kettlitz

Dr. J. Kleiner

Dr. A. Knaap

Dr. M. Knowles

Dr. I. Knudsen

Prof. R. Kroes

Dr. P. Krykant

Dr. J. Larsen

Dr. D. Liem

Dr. M. Lützow

Dr. D. Mantelis

Mrs. J. Martinez Sanchez

Dr. S. Mayer

Dr. B. Meek

Prof. B. Moseley

Dr. D. Müller

Dr. I. Munro

Dr. H. Nordmann

Dr. J. O'Brien

Dr. S. Page

Dr. G. Pascal

Prof. P. Peters

Prof. F. Pfannkuch

Dr. I. Pratt

Mrs. P. Raunemaa

Prof. A. Renwick

Dr. J. Schlatter

Dr. C. Simoneau

Dr. M. Smith

Prof. A. Somogyi

Dr. W. Stargel

Prof. L. Szponar

Ms. A. Takei

Dr. A. Tritscher

Ir. S. Tuijtelaars

Prof. V. Tutelyan

Prof. P. Van den Brandt

Dr. W. Vas Dias

Dr. P. Verger

Dr. A. Wadge

Prof. R. Walker

Dr. G. Würtzen
US Department of Agriculture/ Food Safety and Inspection Service (USDA/FSIS)

University of Münster

The European Consumers Organisation (BEUC)

ILSI Europe

National Institute of Public Health and Environmental Protection (RIVM)

Coca-Cola

Danish Veterinary and Food Administration

IRAS - University of Utrecht

Masterfoods

Danish Veterinary and Food Administration

European Commission - DG SANCO

Food and Agricultural Organization (FAO)

General Chemical State Laboratory

Confederation of the food and drink industries of the EU (CIAA)

Red Bull

Health Canada

University of Reading

Procter \& Gamble

CanTox Health Sciences International

Ajinomoto

Groupe Danone

World Health Organization (WHO)

National Institute for Agricultural Research (INRA)

Inspectorate for Health Protection and Veterinary Public Health

F. Hoffman-La Roche

Food Safety Authority Ireland

National Food Agency

University of Southampton

Swiss Federal Office of Public Health

European Commission Joint Research Centre - Institute for Health and Consumer

Protection

Unilever

European Commission- DG SANCO

NutraSweet

National Food and Nutrition Institute

ILSI Japan

Nestlé

ILSI Europe

National Nutrition Institute

University of Maastricht

Seven Seas

National Institute for Agricultural Research (INRA)

Food Standards Agency

University of Surrey

Coca-Cola Nordic \& Baltics
USA

$\mathrm{D}$

B

B

NL

B

DK

NL

UK

DK

B

I

GR

B

A

CDN

UK

D

CDN

$\mathrm{CH}$

$\mathrm{F}$

$\mathrm{CH}$

$\mathrm{F}$

NL

$\mathrm{CH}$

IRL

FIN

UK

$\mathrm{CH}$

I

NL

B

USA

PL

JPN

$\mathrm{CH}$

B

RUS

NL

UK

F

UK

UK

DK

\section{References}

Advisory Committee on Novel Foods and Processes, 1989. Advisory Committee on Novel Foods and Processes Annual Report, 1989. Department of Health, Ministry of Agriculture, Fisheries and Food, London. pp. 7-8.

Advisory Committee on Novel Foods and Processes, 1991. Guidelines on the Assessment of Novel Foods and Processes. Department of Health Report on Health and Social Subjects, p. 38.

Advisory Committee on Novel Foods and Processes, 1992. Advisory Committee on Novel Foods and Processes Annual Report 1992. Department of Health, Ministry of Agriculture, Fisheries and Food, London, Ohmic heating, p. 8.

Alpha-tocopherol, Beta Carotene Cancer Prevention Study Group,
1994. The effect of vitamin $\mathrm{E}$ and beta carotene on the incidence of lung cancer and other cancers in male smokers. New England Journal of Medicine 330, 1029-1035.

Arnold, S.F., Klotz, D.M., Collins, B.M., Vonier, P.M., Guillette, L.J., McLachlan, J.A., 1996. Synergistic activation of estrogen receptor with combinations of environmental chemicals. Science 272, 1489-1492.

Ashby, J., Lefevre, P.A., Odum, J., Harris, C.A., Routledge, E.J., Sumpter, J.P., 1997. Synergy between synthetic oestrogens? Nature $385,494$.

Baldwin, J.K., Hoover, B.K., 1991. Quality assurance and quality control in toxicology: how might these concepts apply to epidemiology? Journal of Occupational Medicine 33, 1247-1249.

Barlow, S.M., Greig, J.B., Bridges, J.W., Carere, A., Carpy, A.J.M., 
Galli, C.L., Kleiner, J., Knudsen, I., Koëter, H.B.W.M., Levy, L.S., Madsen, C., Mayer, S., Narbonne, J.-F., Pfannkuch, F., Prodanchuk, M.G., Smith, M.R., Steinberg, P., 2002. Hazard identification by methods of animal-based toxicology. Food and Chemical Toxicology $40(2 / 3), 145-191$.

Becher, H., Steindorf, K., Flesch-Janys, D., 1998. Quantitative cancer risk assessment for dioxins using an occupational cohort. Environmental Health Perspectives 106, 663-670.

Beck, B.D., Conolly, R.B., Dourson, M.L., Guth, D., Hattis, D., Kimmel, C., Lewis, S.C., 1993. Improvements in quantitative noncancer risk assessment. Sponsored by the Risk Assessment Specialty Section of the Society of Toxicology. Fundamental and Applied Toxicology 20, 1-14.

Buss, N.E., Tembe, E.A., Prendergast, B.D., Renwick, A.G., George, C.F., 1994. The teratogenic metabolites of vitamin A in women following supplements and liver. Human and Experimental Toxicology 13, 33-43.

Calabrese, E.J., 1985. Uncertainty factors and interindividual variation. Regulatory Toxicology and Pharmacology 5, 190-196.

Cheeseman, M.A., Machuga, E.J., Bailey, A.B., 1999. A tiered approach to threshold of regulation. Food and Chemical Toxicology $37,387-412$.

Codex Committee on Pesticide Residues, 2001. Report of the Thirty-third Session of the CCPR, The Hague, 2-7 April 2001, ALINORM 01/24A. Collaborative Group on Hormonal Factors in Breast Cancer, 2002. Alcohol, tobacco and breast cancer-collaborative reanalysis of individual data from 53 epidemiological studies, including 58515 women with breast cancer and 95067 women without the disease. British Journal of Cancer 87, 1234-1245.

Commission of the European Communities, 1987. Council Directive 87/18/EEC of 18 December 1986 on the harmonisation of laws, regulations and administrative provisions relating to the application of the principles of good laboratory practice and the verification of their applications for tests on chemical substances. Official Journal of the European Communities No. L 15 (17.1.1987), 29.

Commission of the European Communities, 1988. Council Directive 88/320/EEC of 9 June 1988 on the inspection and verification of Good Laboratory Practice (GLP). Official Journal of the European Communities No. L 145 (11.6.1988), 35.

Commission of the European Communities, 2000. Communication from the Commission on the Precautionary Principle. Brussels 2.2.2000. $\operatorname{COM}(2000) .1$ final.

Committee on Toxicity of Chemicals in Food, Consumer Products and the Environment, 2001. Annual Report of the Committees on Toxicity, Mutagenicity and Carcinogenicity of Chemicals in Food, Consumer Products and the Environment. Food Standards Agency/ Department of Health, published in August 2002.

Committee on Toxicity of Chemicals in Food, Consumer Products and the Environment, 2002. Risk Assessment of Mixtures of Pesticides and Similar Substances. Food Standards Agency, London. (Available at http://www.food.gov.uk/science/our advisors/toxicity/ COTwg/wigramp/).

Cramer, G.M., Ford, R.A., Hall, R.L., 1978. Estimation of toxic hazard - a decision tree approach. Food and Cosmetics Toxicology $16,255-276$

Dorne, J.-L.C.M., Walton, K., Renwick, A.G., 2001a. Uncertainty factors for chemical risk assessment. Human variability in the pharmacokinetics of CYP1A2 probe substrates. Food and Chemical Toxicology 39, 681-696.

Dorne, J.-L.C.M., Walton, K., Renwick, A.G., 2001b. Human variability in glucuronidation in relation to uncertainty factors for risk assessment. Food and Chemical Toxicology 39, 1153-1173.

Dorne, J.-L.C.M., Walton, K., Slob, W., Renwick, A.G., 2002. Human variability in polymorphic CYP2D6 metabolism: is the kinetic default uncertainty factor adequate? Food and Chemical Toxicology 40, 1633-1656.

Dorne, J.-L.C.M., Walton, K., Renwick, A.G., 2003. Polymorphic CYP2C19 and N-acetylation: human variability in kinetics and pathway-related uncertainty factors. Food and Chemical Toxicology 41, 225-245.

Dourson, M.L., Stara, J.F., 1983. Regulatory history and experimental support of uncertainty (safety) factors. Regulatory Toxicology and Pharmacology 3, 224-238.

Dourson, M.L., Felter, S.P., Robinson, D., 1996. Evolution of science-based uncertainty factors in noncancer risk assessment. Regulatory Toxicology and Pharmacology 24, 108-120.

Duffus, J.H., 1993. Glossary for chemists of terms used in toxicology (International Union of Pure and Applied Chemistry recommendations 1993). Pure and Applied Chemistry 65, 2003-2122.

Duffus, J.H., 2000. Some terms used in toxicology, chemical safety and allied subjects. Department of Community Health Sciences, University of Edinburgh Medical School, Edinburgh, Scotland. (Available at http://www.med.ed.ac.uk/HEW/tox/glossall.html; last updated: April 28, 2000; downloaded November 9, 2002).

Dybing, E., Sanner, T., Roelfzema, H., Kroese, D., Tennant, R.W., 1997. T25: a simplified carcinogenic potency index: description of the system and study of correlations between carcinogenic potency and species/site specificity and mutagenicity. Pharmacology and Toxicology 80, 272-279.

Dybing, E., Doe, J., Groten, J., Kleiner, J., O'Brien, J., Renwick, A.G., Schlatter, J., Steinberg, P., Tritscher, A., Walker, R., Younes, M., 2002. Hazard characterisation of chemicals in food and diet: dose response, mechanisms and extrapolation issues. Food and Chemical Toxicology 40 (2/3), 237-282.

Edler, L., Poirier, K., Dourson, M., Kleiner, J., Mileson, B., Nordmann, H., Renwick, A., Slob, W., Walton, K., Würtzen, G., 2002. Mathematical modelling and quantitative methods. Food and Chemical Toxicology 40 (2/3), 283-326.

Eisenbrand, G., Pool-Zobel, B., Baker, V., Balls, M., Blaauboer, B.J., Boobis, A., Carere, A., Kevekordes, S., Lhuguenot, J.-C., Pieters, R., Kleiner, J., 2002. Methods of in vitro toxicology. Food and Chemical Toxicology 40 (2/3), 193-236.

Environmental Protection Agency, 1997. EPA Terms of Environment. U.S. Environmental Protection Agency, Office of Communications, Education, and Public Affairs, Washington, DC. (Available at http://www.epa.gov/OCEPAterms/; last updated May 13, 1998; downloaded May 5, 2000).

Environmental Protection Agency, 1999a. Standard Operating Procedures (SOPs) for the Use of the FQPA Factor. US Environmental Protection Agency, Docket Number OPP-06610.

(Available at http://www.epa.gov/oppfead1/trac/science).

Environmental Protection Agency, 1999b. Guidelines for Carcinogen Risk Assessment. NCEA-F-0644. July 1999. Review Draft. Risk Assessment Forum, US Environmental Protection Agency, Washington DC. (Available at http://www.epa.gov).

Environmental Protection Agency, 2001. General Principles for Performing Aggregate Exposure and Risk Assessments. US Environmental Protection Agency, Docket Number OPP-00732. (Available at http://www.epa.gov/oppfead1/trac/science).

Environmental Protection Agency, 2002. Guidance on Cumulative Risk Assessment of Pesticide Chemicals that have a Common Mechanism of Toxicity. US Environmental Protection Agency, Docket Number OPP-00610.

(Available at http://www.epa.gov/oppfead1/trac/science).

European Commission, 1993. Reports of the scientific committee for food (thirty-first series). Nutrient and energy intakes for the European Community. (Opinion expressed on 11 December 1992.) Directorate-General Industry. Commission of the European Communities, Luxembourg.

European Commission, 2000. Report of the EC Scientific Steering Committee's Working Group on Harmonisation of Risk Assessment Procedures in the Scientific Committees advising the European Commission in the area of human and environmental health- 26-27 October 2000. Part 2, Appendix 1- Glossary of Terms. (Available at http://europa.eu.int/comm/food/fs/sc/ssc/out82_en.html). 
European Commission, 2002. Regulation (EC) No 178/2002 of the European parliament and of the council of 28 January 2002 laying down the general principles and requirements of food law, establishing the European food safety authority and laying down procedures in matters of food safety. Official Journal of the European Commission: L31/1-24.

Fletcher, R.H., Fletcher, S.W., Wagner, E.H., 1988. Clinical Epidemiology; the essentials. Williams \& Wilkins, Baltimore.

Food and Drug Administration, 1993. Food additives; threshold of regulation for substances used in food-contact articles. Federal Register 58, 52719-52727.

Food and Drug Administration, 1995. Food additives: threshold of regulation for substances used in food-contact articles; final rule. Federal Register 60, 36582-36596.

Food and Drug Administration, 1997. Federal Register 62(90, May 9), 25691-25709. (Available at http://www.fda.gov/gcp/).

Food Safety in Europe, 2002. Food Safety in Europe (FOSIE): Risk Assessment of Chemicals in Food and Diet, Barlow, S., Dybing, E., Edler, L., Eisenbrand, G., Kroes, R., van den Brandt, P. (Eds.). Food and Chemical Toxicology 40(2/3), pp. 137-427.

German Professional Society of Epidemiology, 2000. Leitlinien und Empfehlungen zur Sicherung von Guter Epidemiologischer Praxis (GEP) [Guidelines and recommendations for ensuring good epidemiological practice (GEP)]. Gesundheitswesen 62, 295-302.

Gold, L.S., Manley, N.B., Ames, B.N., 1992. Extrapolation of carcinogenicity between species: qualitative and quantitative factors. Risk Analysis 12, 579-588.

Goldman, L.R., 2001. Epidemiology in the regulatory arena. American Journal of Epidemiology 154 (Supplement), S18-S26.

Groten, J.P., Schoen, E.D., van Bladeren, P.J., Kuper, F.C.F., van Zorge, J.A., Feron, V.J., 1997. Subacute toxicity of a mixture of nine chemicals in rats: detecting interactive effects with a fractionated two-level factorial design. Fundamental and Applied Toxicology 36, 15-29.

Groten, J.P., Butler, W., Feron, V.J., Kozianowski, G., Renwick, A.G., Walker, R., 2000. An analysis of the possibility for health implications of joint actions and interactions between food additives. Regulatory Toxicology and Pharmacology 31, 77-91.

Hayes, A.W., 2001. Glossary. Principles and Methods of Toxicology, fourth edition. Wallace, Hayes, A. Taylor and Francis Publishers, Philadelphia, pp. 1807-1835.

Health Canada, 1994. Guidelines for the assessment of novel foods.

Hertz-Picciotto, I., 1995. Epidemiology and quantitative risk assessment: a bridge from science to policy. American Journal of Public Health 85, 484-491.

Hertz-Picciotto, I., Holtzman, D.A., 1989. Issues in conducting a cancer risk assessment using epidemiologic data: arsenic as a case study. Experimental Pathology 37, 219-223.

Hertz-Picciotto, I., Hu, S.W., 1994. Contribution of cadmium in cigarettes to lung cancer: an evaluation of risk assessment methodologies. Archives of Environmental Health 49, 297-302.

Institute of Medicine, 1997. Dietary Reference Intakes for Calcium, Phophorus, Magnesium, Vitamin D, and Fluoride. Standing Committee on the Scientific Evaluation of Dietary Reference Intakes. Food and Nutrition Board, Institute of Medicine. National Academy Press, Washington DC, USA.

International Life Sciences Institute, 1992. Similarities and differences between children and adults: implications for risk assessment. ILSI Europe, Brussels.

International Life Sciences Institute, 1999a. Significance of excursions of intake above the acceptable daily intake. Report of a Workshop held in April 1998. ILSI Europe, Brussels.

International Life Sciences Institute, 1999b. ILSI Europe workshop on the significance of excursions of intake above the acceptable daily intake (ADI). Barlow, S., Pascal, G., Larsen, J.C., Richold, M. (Eds.). Regulatory Toxicology and Pharmacology 30(2), Part 2 of 2 Parts, S1-S121.
International Life Sciences Institute, 2000a. Threshold of Toxicological Concern for Chemical Substances Present in the Diet. Report of a Workshop held in October 1999. ILSI Europe Report Series 2000. ILSI Europe, Brussels.

International Life Sciences Institute, 2000b. The Acceptable Daily Intake: A Tool for Ensuring Food Safety. By Diane Benford. ILSI Europe Concise Monograph Series. ILSI Europe, Brussels.

International Programme on Chemical Safety, 2001a. Guidance document for the use of data in development of chemical-specific adjustment factors (CSAFs) for interspecies differences and human variability in dose /concentration response assessments. Document number $\mathrm{WHO} / \mathrm{IPCS} / 01.4$.

(Available at http://www.ipcsharmonize.org).

International Programme on Chemical Safety, 2001b. Glossary of key exposure assessment terms. Prepared by the Exposure Terminology Subcommittee of the IPCS Exposure Assessment Planning Workgroup for the Harmonization of Approaches to the Assessment of Risk from Exposure to Chemicals. November 1, 2001.

Joint Expert Committee on Food Additives, 1993. Evaluation of certain food additives and contaminants. Safety evaluation of flavouring agents. Forty-first report of the Joint FAO/WHO Expert Committee on Food Additives. WHO Technical Report Series 837. Geneva: World Health Organization.

Joint Expert Committee on Food Additives, 1995. Evaluation of certain food additives and contaminants. Safety evaluation of flavouring agents. Forty-fourth report of the Joint FAO/WHO Expert Committee on Food Additives. WHO Technical Report Series 859. Geneva: World Health Organization.

Joint Expert Committee on Food Additives, 1999. Evaluation of certain food additives and contaminants. Forty-ninth report of the Joint FAO/WHO Expert Committee on Food Additives. WHO Technical Report Series 884. Geneva: World Health Organization.

Joint Expert Committee on Food Additives, 2000. Evaluation of certain food additives. Fifty-first report of the Joint FAO/WHO Expert Committee on Food Additives. WHO Technical Report Series 891. Geneva: World Health Organization.

Joint FAO/WHO Meeting on Pesticide Residues, 2002. Report of the 2002 joint FAO/WHO meeting on pesticide residues, Rome, 16-25 September 2002. (Available at

http://www.fao.org/ag/agp/agpp/pesticid/jmpr/pm_jmpr.htm).

Jonker, D., Woutersen, R.A., Feron, V.J., 1996. Toxicity of mixtures of nephrotoxicants with similar or dissimilar mode of action. Food and Chemical Toxicology 34, 1075-1082.

Knudsen, L.E., Loft, S.H., Autrup, H., 2001. Risk assessment: the importance of genetic polymorphisms in man. Mutation Research 482, 83-88.

Kroes, R., Galli, C., Munro, I., Schilter, B., Tran, L.-A., Walker, R., Würtzen, G., 2000. Threshold of toxicological concern for chemical substances present in the diet: a practical tool for assessing the need for toxicity testing. Food and Chemical Toxicology 38, 255-312.

Kroes, R., Müller, D., Lambe, J., Löwik, M.R.H., van Klaveren, J., Kleiner, J., Massey, R., Mayer, S., Urieta, I., Verger, P., Visconti, A., 2002. Assessment of intake from the diet. Food and Chemical Toxicology 40 (2/3), 327-385.

Lambe, J., Kearney, J., Leclerc, C., Berardi, D., Zunft, H.F., Sulzer, S., De Henauw, S., De Volder, M., Lamberg-Allardt, C.J., Karkkainen, M.U., Dunne, A., Gibney, M.J., 2000. Enhancing the capacity of food consumption surveys of short duration to estimate long-term consumer-only intakes by combination with a qualitative food frequency questionnaire. Food Additives and Contaminants 17, 177-187.

Lovell, D.P., Thomas, G., 1996. Quantitative risk assessment and the limitations of the linearized multistage model. Human and Experimental Toxicology 15, 87-104.

Life Sciences Research Office, 1999. The LSRO Report on Alternative and Traditional Models for Safety Evaluation of Food Ingredients. Raiten, D.J. (Ed.). Life Sciences Research Office, American Society for Nutritional Sciences, Bethesda MD, USA. 
Lutz, W.K., 1998. Dose-response relationships in chemical carcinogenesis: superposition of different mechanisms of action, resulting in linear-nonlinear curves, practical thresholds, J-shapes. Mutation Research 405, 117-124.

Malmfors, T., Di Marco, P. and Savolainen, K., 2001. Good evaluation practice. Guidelines proposal.

(Available at http://www.toxicology.org/iutox/GEP.htm).

McLachlan, J.A., 1997. Synergistic effect of environmental estrogens: Report withdrawn. Science 277, 462-463.

Mottram, D.S., Wedzicha, B.L., Dodson, A.T., 2002. Acrylamide is formed in the Maillard reaction. Nature 419, 448-449.

Munro, I.C., 1990. Safety Assessment Procedures for Indirect Food Additives: an overview. Report of a workshop. Regulatory Toxicology and Pharmacology 12, 2-12.

Munro, I.C., Ford, R.A., Kennepohl, E., Sprenger, J.G., 1996. Correlation of structural class with no-observed effect levels: a proposal for establishing a threshold of concern. Food and Chemical Toxicology 34, 829-867.

Omenn, G.S., Goodman, G.E., Thornquist, M.D., Balmes, J., Cullen, M.R., Glass, A., Keogh, J.P., Meyskens, F.L., Valanis, B., Williams, J.H., Barnhart, S., Cherniack, M.G., Brodkin, C.A., Hammar, S., 1996. Risk factors for lung cancer and for intervention effects in CARET, the beta-carotene and retinol efficacy trial. Journal of the National Cancer Institute 88, 1550-1559.

Organisation for Economic Co-operation and Development, 1998. OECD Guideline for the Testing of Chemicals. Updated Guideline 408, adopted 21 September 1998. OECD, Paris.

Organisation for Economic Co-operation and Development, 2000. OECD Guidelines for the Testing of Chemicals. Eleventh Addendum, subsequent revisions. OECD, Paris.

Organisation for Economic Co-operation and Development, 2002. Chemical definitions of generic terms used in hazard and risk assessment. Joint OECD/IPCS Project on the Harmonisation of Hazard/Risk Assessment Terminology. Draft Report January 2002.

Pesticides Safety Directorate, 2001. Data Requirements Handbook. (Available at http://www.pesticides.gov.uk/applicant/registration_ guides/data_reqs_handbook/datareqhandbook.pdf).

Rajapakse, N., Ong, D., Kortenkamp, A., 2001. Defining the impact of weakly estrogenic chemicals on the action of steroidal estrogens. Toxicological Sciences 60, 296-304.

Renwick, A.G., 1993. Data-derived safety factors for the evaluation of food additives and environmental contaminants. Food Additives and Contaminants 10, 275-305.

Renwick, A.G., 1995. The use of an additional safety or uncertainty factor for nature of toxicity in the estimation of acceptable daily intake and tolerable daily intake values. Regulatory Toxicology and Pharmacology 22, 250-261.

Renwick, A.G., 1999a. Incidence and severity in relation to magnitude of intake above the ADI or TDI: use of critical effect data. Regulatory Toxicology and Pharmacology 30, S79-S86.

Renwick, A.G., 1999b. Duration of intake above the ADI/TDI in relation to toxicodynamics and toxicokinetics. Regulatory Toxicology and Pharmacology 30, S69-S78.

Renwick, A.G., 2000. The use of safety or uncertainty factors in the setting of acute reference doses. Food Additives and Contaminants 17, 627-635.

Renwick, A.G., 2002. Pesticide residue analysis and its relationship to hazard characterisation (ADI/ARfD) and intake estimations (NEDI/NESTI). Pest Management Science 58, 1073-1082.

Renwick, A.G., Lazarus, N.R., 1998. Human variability and noncancer risk assessment-an analysis of the default uncertainty factor. Regulatory Toxicology and Pharmacology 27, 3-20.

Renwick, A.G., Walton, K., 2001. The use of surrogate endpoints to assess potential toxicity in humans. Toxicology Letters 120, 97-110.

Rushton, L., 2000. Reporting of occupational and environmental research: use and misuse of statistical and epidemiological methods. Occupational and Environmental Medicine 57, 1-9.
Sanner, T., Dybing, E., Willems, M.I., Kroese, E.D., 2001. A simple method for quantitative risk assessment of non-threshold carcinogens based on the dose descriptor T25. Pharmacology and Toxicology $88,331-341$.

Schantz, S.L., 1996. Developmental neurotoxicity of PCBs in humans: what do we know and where do we go from here? Neurotoxicology and Teratology 18, 217-227.

Scientific Committee for Food, 1984. Commission of the European Communities. Reports of the Scientific Committee for Food of the European Community. Fourteenth series. Essential requirements of infant formulae and follow-up milks based on cows' milk proteins. Commission of the European Communities, Luxembourg.

Scientific Committee for Food, 1993. Commission of the European Communities. Reports of the Scientific Committee for Food of the European Community (Thirty-first series). Nutrient and energy intakes for the European Community. (Opinion expressed on 11 December 1992.) Directorate-General Industry. Commission of the European Communities, Luxembourg.

Scientific Committee for Food, 1997. Recommendations concerning the scientific aspects of information necessary to support applications for placing on the market of novel foods and novel food ingredients. Part I. Opinion expressed on 7 June 1996. Scientific Committee for Food (Thirty-ninth Series). Part II. Opinion expressed on 13 December 1996. Scientific Committee for Food (Fortieth Series). Part III. Opinion expressed on 13 December 1996. Scientific Committee for Food (Fortieth Series).

Scientific Committee on Food, 1998. Opinion on the applicability of the ADI (Acceptable Daily Intake) for food additives to infants, expressed on 17/09/1998. Scientific Commitee on Food. Annex 1 to Doc XXIV/2/10/98.

Scientific Committee on Food, 2000a. Guidelines for presentation of an application for assessment of a substance to be used in food contact materials prior to its authorisation. Opinion of the Scientific Committee on Food. Annex III to the Minutes of the 124th Plenary Meeting of the Scientific Committee on Food held on 21-22 November 2000, Brussels.

Scientific Committee on Food, 2000b. Opinion of the Scientific Committee on Food on the risk assessment of dioxins amd dioxin-like PCBs in food. Adopted on 22 November 2000. (Available at http://europa.eu.int/comm/food/fs/sc/scf/outcome_en.html).

Scientific Committee on Food, 2000c. Guidelines of the Scientific Committee on Food for the development of tolerable upper intake levels for vitamins and minerals. Opinion expressed on 19 October 2000. (Available at http://europa.eu.int/comm/food/fs/sc/scf/out80a_en.pdf).

Scientific Committee on Food, 2001. Opinion of the Scientific Committee on Food on the risk assessment of dioxins and dioxin-like PCBs in food (update based on the new scientific information available since the adoption of the SCF opinion on 22 November 2000). Adopted by the SCF on 30 May 2001. (Available at http://europa.eu.int/comm/food/fs/sc/scf/outcome_en.html).

Scientific Committee on Food, 2002a. A general view on the long-term effects of the intake of elevated levels of phytosterols from multiple dietary sources, with particular attention to the effects on b-carotene. Opinion expressed on 26 September 2002. (Available at: http://www.europa.eu.int/comm/food/fs/sc/scf/index_en.html).

Scientific Committee on Food, 2002b. Opinion of the Scientific Committee on Food on the risks to human health of Polycyclic Aromatic Hydrocarbons in food. Opinion expressed on 4 December 2002. (Available at http://www.europa.eu.int/comm/food/fs/sc/scf/index_en.html).

Shore, R.E., Iyer, V., Altshuler, B., Pasternack, B.S., 1992. Use of human data in quantitative risk assessment of carcinogens: impact on epidemiologic practice and the regulatory process. Regulatory Toxicology and Pharmacology 15, 180-221.

Smith, M., 2002. Food Safety in Europe (FOSIE): risk assessment of chemicals in food and diet: overall introduction. Food and Chemical Toxicology 40 (2/3), 141-144. 
Stayner, L., Bailer, A.J., Smith, R., Gilbert, S., Rice, F., Kuempel, E., 1999. Sources of uncertainty in dose-response modeling of epidemiological data for cancer risk assessment. Annals of the New York Academy of Sciences 895, 212-222.

Steenland, K., Deddens, J., Piacitelli, L., 2001. Risk assessment for 2,3,7,8-tetrachlorodibenzo-p-dioxin (TCDD) based on an epidemiologic study. American Journal of Epidemiology 154, 451-458.

Swedish National Food Authority, 2002. Acrylamide is formed during the prepartion of food and occurs in many food stuffs. (Available at http://www2.slv.se).

Tajima, O., Schoen, E.D., Feron, V.J., Groten, J.P., 2002. Statistically designed experiments in a tiered approach to screen mixtures of Fusarium mycotoxins for possible interactions. Food and Chemical Toxicology 40, 685-695.

van den Brandt, P., Voorrips, L., Hertz-Picciotto, I., Shuker, D., Boeing, H., Speijers, G., Guittard, C., Kleiner, J., Knowles, M., Wolk, A., Goldbohm, A., 2002. The contribution of epidemiology to risk assessment of chemicals in food and diet. Food and Chemical Toxicology 40 (2/3), 387-424.

Van Landingham, C.B., Allen, B.C., Shipp, A.M., Crump, K.S., 2001. Comparison of the EU T25 single point estimate method with benchmark dose response modeling for estimating potency of carcinogens. Risk Analysis 21, 641-656.

van Leeuwen, C.L., Hermens, J. (Eds.), 1996. Risk Assessment of Chemicals: An Introduction. Kluwer Academic Publishers, Dordrecht, Boston and London.

Vermeire, T., Stevenson, H., Peiters, M.N., Rennen, M., Slob, W., Hakkert, B.C., 1999. Assessment factors for human health risk assessment: a discussion paper. Critical Reviews in Toxicology 29, 439-490.

Vineis, P., Schulte, P., McMichael, A.J., 2001. Misconceptions about the use of genetic tests in populations. Lancet 357, 709-712.

Vrijens, B., De Henauw, S., Dewettinck, K., Talloen, W., Goeyens, L., De Backer, G., Willems, J.L., 2002. Probabilistic intake assessment and body burden estimation of dioxin-like substances in background conditions and during a short food contamination episode. Food Additives and Contaminants 19, 687-700.

Wade, M.G., Desaulniers, D., Leingartner, K., Foster, W.G., 1997. Interactions between endosulfan and dieldrin on estrogen-mediated processes in vitro and in vivo. Reproductive Toxicology 11, 791798.

Walton, K., Dorne, J.-L.C.M., Renwick, A.G., 2001. Default factors for interspecies differences in the major route of xenobiotic elimination. Human and Ecological Risk Assessment 7, 181-201.

Wilkinson, C.F., Christoph, G.R., Julien, E., Kelley, J.M., Kronenberg, J., McCarthy, J., Reiss, R., 2000. Assessing the risks of exposures to multiple chemicals with a common mechanism of toxicity: how to cumulate? Regulatory Toxicology and Pharmacology 31 (1), $30-43$.

World Health Organization, 1987. Principles for the Safety Assessment of Food Additives and Contaminants in Food. Environmental Health Criteria 70. International Programme on Chemical Safety, Geneva.

World Health Organization, 1990. Principles for the Toxicological Assessment of Pesticide Residues in Food. Environment Health Criteria 104. International Programme on Chemical Safety, Geneva.

World Health Organization, 1994. Assessing Human Health Risks of Chemicals: Derivation of Guidance Values for Health-based Exposure Limits. Environment Health Criteria 170. International Programme on Chemical Safety, Geneva.

World Health Organization, 1997. Guidelines for predicting dietary intake of pesticide residues. Programme of Food Safety and Food Aid. World Health Organization, Geneva.

World Health Organization, 1999. Principles for the Assessment of Risks to Human Health from Exposure to Chemicals. International Programme on Chemical Safety. Environment Health Criteria 210. WHO, Geneva.
World Health Organization, 2002. Safety evaluation of certain food additives and contaminants. WHO Food Additive Series 48, 451658. World Health Organization, Geneva.

Zito, R., 2001. Low doses and thresholds in genotoxicity: from theories to experiments. Journal of Experimental and Clinical Cancer Research 20, 315-325.

\section{Glossary}

Term:

Acceptable

daily intake:

Acceptable risk: The probability of suffering an adverse effect that will be tolerated by an individual, group, system or population. The acceptability of the risk depends on scientific data, social, economic, and political factors, and on the perceived benefits arising from exposure to an agent (Organisation for Economic Co-operation and Development, 2002).

Accumulation: Repeated doses of an agent which results in progressive increase in the concentration of that agent in an organism, organ or tissue leading to potentially more marked effects with successive doses (Duffus, 2000).

Acute toxicity: Adverse effects occurring within a short time of administration of a single dose of an agent, or immediately following short or continuous exposure, or multiple doses over 24 hours or less (Duffus, 2000).

Additivity: $\quad$ Consequence of two or more agents acting together. It is the simple sum of the effects acting independently (Duffus, 2000).

Acute reference The estimated amount of a substance dose: in food or drinking-water, expressed on a body weight basis, that can be 
ingested over 24 hours or less, without appreciable health risk to the consumer on the basis of all the known facts at the time of the evaluation (Joint Meeting on Pesticide Residues, 2002).

$A D M E$ :

Adverse effect: Absorption, distribution, metabolism, and excretion. The processes that determine the disposition and fate of an administered agent (Hayes, 2001).

Change in the morphology, physiology, growth, development or life span of an organism, system or (sub) population that results in an impairment of functional capacity, an impairment of the capacity to compensate for additional stress, or an increase in susceptibility to other external influences (Organisation for Economic Co-operation and Development, 2002).

Aggregate risk assessment:

Term introduced under the Food Quality Protection Act 1996 in the USA, to describe risk assessment taking all sources of intake of a given pesticide into account (Committee on Toxicity of Chemicals in Food, Consumer Products and the Environment, 2002).

Antagonism: Combined effect of two or more agents that is smaller than the solitary effect of any one of those agents (Duffus, 1993).

Assessment: $\quad$ Evaluation or appraisal of analysis of facts and inference of possible consequences concerning a particular object or process (Organisation for Economic Co-operation and Development, 2002).

Assessment Numerical adjustment used to extraFactor: $\quad$ polate from dose-response relationships to estimate the agent exposure below which an adverse effect is not likely to occur (Organisation for Economic Co-operation and Development, 2002). Related terms: Uncertainty Factor and Safety Factor.

Bench Mark The lower confidence limit on a dose Dose (BMD): associated with a specified level of response (Hayes, 2001).

Bioavailability: The fraction or percentage of the administered dose what enters the general circulation as the parent compound (Hayes, 2001).

Biomarker: Measurement that indicates exposure to an agent, the effect of such expo- sure, or susceptibility to effect of such an exposure (Hayes, 2001).

Carcinogenicity: Process of induction of malignant neoplasms by an agent (Duffus, 1993).

Case Control A study in which the past histories of Study: those with a specific disease (the cases) are compared with those who do not have the disease (the controls) (Hayes, 2001).

Chronic Adverse effects occurring as a result toxicity: $\quad$ of repeated dosing of an agent on a daily basis, or exposure to that agent, for a large part of an organism's lifespan, usually more than 50\% (Duffus, 2000).

Cohort Study: Method of epidemiology study in which a group of people (a cohort) is assembled, none of whom has experienced the outcome of interest. On entry to the study, people in the cohort are classifed according to their observed exposure and other characteristics that might be related to outcome. These people are then followed over time to see which of them experience the outcome. In a prospective cohort study, the exposure is defined before the outcome events occur. This ensures that the time relationship between exposure and outcome is appropriate; the follow-up procedures allow direct measurement of the incidence rate of the outcome(s) in each of the exposure groups studied (Fletcher et al., 1988).

Confounder: An alternative cause for the disease in question that is unequally distributed among those exposed and nonexposed to the putative agent of interest (Hayes, 2001).

Cumulative risk Term introduced under the Food assessment: Quality Protection Act 1996 in the USA, to describe risk assessment taking intake of more than one pesticide into account (Committee on Toxicity of Chemicals in Food, Consumer Products and the Environment, 2002).

Cumulative The sum of exposures of an organism, exposure: system or (sub) population to an agent over a period of time (Environmental Protection Agency, 1997).

Dietary reference Reference values that can be used for intakes (DRI's): planning and assessing diets for healthy populations and for many other purposes (Institute of Medicine, 1997). 
Dose:

Dose-response assessment:

Dose-response curve:

Dose-response relationship:

\section{Ecological Risk} Assessment:

Effect:

Effect

Assessment:

Expert judgement:

Exposure:

Exposure assessment:
Total amount of an agent administered to, taken or absorbed by an organism, system or (sub) population (Organisation for Economic Cooperation and Development, 2002).

The determination of the relationship between the magnitude of exposure to risk source(s) and the magnitude or frequency and/or severity of associated adverse effect(s) (European Commission, 2000).

Graphical presentation of a doseresponse relationship (van Leeuwen and Hermens, 1996).

Relationship between the amount of an agent administered to, taken or absorbed by an organism, system or (sub) population and the change developed in that organism, system or (sub) population in reaction to the agent (Organisation for Economic Cooperation and Development, 2002).

Risk Assessment process that evaluates the likelihood that adverse ecological effects may occur or are occurring as result of exposure to one or more stressors (Organisation for Economic Co-operation and Development, 2002).

Change in the state or dynamics of an organism, system or (sub) population caused by the action of an agent (Organisation for Economic Cooperation and Development 2002).

Combination of analysis and inference of possible consequences of the exposure to a particular agent based on knowledge of the dose-effect relationship associated with that agent in a specific target organism, system or (sub) population (Organisation for Economic Co-operation and Development, 2002).

Opinion of an authoritative person on a particular subject (Organisation for Economic Co-operation and Development, 2002).

Concentration or amount of a particular agent that reaches a target organism, system or (sub) population in a specific frequency for a defined duration (Organisation for Economic Co-operation and Development, 2002).

The process of estimating or measuring the intensity, frequency, and
Exposure

scenario:

Fate:

Food:

Genotoxic:

Good

laboratory

practice:

Good evaluation practice:

Hazard:

Hazard characterisation: evaluation of the nature of the duration of exposure to an agent. Ideally, it describes the sources, pathways, routes, magnitude, duration, and pattern of exposure; the characteristics of the populations exposed; and the uncertainties in the assessment (International Programme on Chemical Safety, 2001b).

A set of conditions or assumptions about sources, exposure pathways, amount or concentrations of agent(s), and exposed organism, system or (sub) population (i.e. numbers, characteristics, habits) used to aid in the evaluation and quantification of exposure(s) in a given situation (Organisation for Economic Cooperation and Development, 2002).

Pattern of distribution of an agent, its derivatives or metabolites in an organism, system or (sub) population or compartment of concern as a result of transport, partitioning, transformation or degradation (Organisation for Economic Co-operation and Development, 2002).

Any substance or product, whether processed, partially processed or unprocessed, intended to be, or reasonably expected to be ingested by humans. "Food" includes drink, chewing gum and any substance, including water, intentionally incorporated into the food during its manufacture, preparation or treatment (European Commission, 2002).

Property of an agent to cause harmful changes to DNA (Duffus, 2000).

Fundamental rules incorporated in national regulations concerning the process of effective organisation and the conditions under which laboratory studies are properly planned, performed, monitored, recorded, and reported (Duffus, 1993).

Principles concerning the effective organisation and the conditions under which risk assessments are properly planned, performed, monitored, recorded and reported (Malmfors et al., 2001)

The potential of a risk source to cause and adverse effect(s)/event(s) (European Commission, 2000).

The quantitative or semi-quantitative 
adverse health effects to humans and/ or the environment following exposure to a risk source(s). This must, where possible, include a doseresponse assessment (European Commission, 2000).

Hazard The identification of a risk source(s) identification: capable of causing adverse effect(s)/event(s) to humans or the environment species, together with a qualitative description of the nature of these effect(s)/event(s) (European Commission, 2000).

Homeostasis: Inherent tendency in an organism toward maintenance of physiological and psychological stability (Duffus, 2000).

Interaction: $\quad$ A result in which chemicals in a mixture produce an increased or different response as compared with the sum of the responses of the individual chemicals present in the mixture (Committee on Toxicity of Chemicals in Food, Consumer Products and the Environment, 2002).

Internal dose: Amount of an agent penetrating the absorption barriers via either physical or biological processes (Hayes, 2001).

In vitro:

Study carried out in isolation from the living organism in an experimental system (Duffus, 2000).

In vivo: $\quad$ Study carried out within the living organism (Duffus, 2000).

Irreversible: Persisting change in structure or function (Duffus, 1993).

Lowest observed Lowest amount or concentration of adverse effect an agent, found by experiment or level ( $L O A E L$ ): observation, that causes an adverse alteration of morphology, functional capacity, growth, development or life span in an organism, system or (sub) population (Duffus, 1993).

Margin of Ratio of the critical no observed exposure adverse effect level (NOAEL) to the (MOE): theoretical, predicted or estimated exposure dose or concentration (Organisation for Economic Cooperation and Development, 2002).

Margin of Ratio of the critical no-observedsafety (MOS): adverse-effect level (NOAEL) to the actual exposure dose or concentration (Environmental Protection Agency, 1997).

Measurement Endpoint:
Measurable ecological characteristic that is related to the valued characteristic chosen as an assessment point
(Organisation for Economic Co-operation and Development, 2002).

No observed Greatest concentration or amount of adverse effect an agent, found by experiment or level (NOAEL): observation, that causes no detectable adverse alteration of morphology, functional capacity, growth, development or life span in an organism, system or (sub) population (Duffus, 2000).

PBTK model: $\quad$ Physiologically based toxicokinetic model: a mechanistic model that describes quantitatively the uptake, distribution, metabolism and excretion of an agent in an organism, system or (sub) population (Hayes, 2001).

Population The intake that is enough for virreference intake tually all healthy people in a group (PRI):

Post-launch monitoring

(PLM): (European Commission, 1993).

Assessment of the amount and pattern of human intake and determination of the nature and degree of expected and unexpected effects after the introduction of a new product on the market (van den Brandt et al., 2002).

Problem Process by which the issues and formulation: questions are defined and the plan for analysing and characterising risk is developed. (This article, see Section 2.1)

\section{Prospective \\ See: Cohort study} (cohort) study: $Q S A R$ :

Quantitative structure-activity Relationship: Quantitative analysis of the dependence of the biological effects of an agent upon its molecular structure (Duffus, 2000).

Reference dose: An estimate of the daily exposure dose that is likely to be without appreciable health effect even if continued exposure occurs over a lifetime (Organisation for Economic Cooperation and Development, 2002). Related term: Acceptable Daily Intake.

Response: Change developed in the state or dynamics of an organism, system or (sub) population in reaction to exposure to an agent (Organisation for Economic Co-operation and Development, 2002).

Risk: The probability and severity of an adverse effect/event occurring to man or the environment following exposure, under defined conditions, to a 
risk source(s) (European Commission, 2000).

Risk analysis: A process consisting of three components: risk assessment, risk management and risk communication (European Commission, 2000).

Risk assessment: A process of evaluation including identification of the attendant uncertainties, of the likelihood and severity of an adverse effect(s)/event(s) occurring to man or the environment following exposure under defined conditions to a risk source(s). A risk assessment comprises hazard identification, hazard characterisation, exposure assessment and risk characterisation (European Commission, 2000).

Risk The quantitative or semi-quantitative characterisation: estimate, including attendant uncertainties, of the probability of occurrence and severity of adverse effect(s)/ event(s) in a given population under defined exposure conditions based on hazard identification, hazard characterisation and exposure assessment (European Commission, 2000). Risk characterisation is the fourth step in the risk assessment process

Risk The interactive exchange of informacommunication: tion and science based opinions concerning risk among risk assessors, risk managers, consumers and other actual or potential stakeholders (European Commission, 2000).

Risk estimation: Quantification of the probability, including attendant uncertainties, that specific adverse effects will occur in an organism, system or (sub) specific effect population due to actual or predicted exposure (Organisation for Economic Co-operation and Development, 2002).

Risk The process of weighing policy altermanagement: natives in the light of the result of a risk assessment and other relevant evaluation and, if required, selecting and implementing appropriate control options (which should, where appropriate, include monitoring/surveillance) (European Commission, 2000). Risk management consists of three parts: risk evaluation; emission and exposure control; risk monitoring.

Risk monitoring: Process of following up the decisions and actions within risk management in order to ascertain that risk containment or reduction with respect to a particular hazard is assured (Organisation for Economic Co-operation and Development, 2002).

Risk source: Agent, medium, commercial/industrial process, procedure or site with the potential to cause and adverse effect(s)/event(s) (European Commission, 2000).

Safety: $\quad$ Practical certainty that adverse effects will not be caused by an agent under defined circumstances (Organisation for Economic Co-operation and Development, 2002).

Safety factor: Reductive factor by which an observed or estimated no observed adverse effect level (NOAEL) concentration or dose is divided to arrive at a criterion or standard that is considered safe or without appreciable risk (Organisation for Economic Cooperation and Development, 2002). Related terms: Uncertainty factor, Assessment factor.

Structure-activity Qualitative analysis of the depenrelationship dence of the biological effects of an (SAR): $\quad$ agent upon its molecular structure Subchronic toxicity: (Duffus, 2000).

The adverse effects occurring as a result of the repeated daily dosing of a chemical to experimental animals for part (not exceeding 10 per cent) of the life span (Organisation for Economic Co-operation and Development 1998).

Threshold: $\quad$ Dose or exposure concentration of an agent below which a stated effect is not observed or expected to occur (Organisation for Economic Cooperation and Development, 2002).

Threshold of Human exposure threshold value for toxicological classes of substances in the diet below concern (TTC): which there is no significant risk to human health (Kroes et al., 2000).

Tolerable daily Analogous to acceptable daily intake. intake (TDI):

Tolerable (daily weekly, monthly) intake:

Estimate of the amount of an agent that can be ingested or absorbed in the period specified, over a life time without appreciable health risk (Duffus, 2000).

Tolerable upper The maximum level of total chronic intake level $(U L)$ : daily intake of a nutrient (from all sources) judged to be unlikely to pose a risk of adverse health effects to 
humans. 'Tolerable intake' in this context connotes what is physiologically tolerable and is a scientific judgement as determined by assessment of risk, i.e. the probablity of an adverse effect occurring at some specified level of exposure. ULs may be derived for various groups in the population (Scientific Committee on Food, 2000c).

Toxicant: An agent capable of causing an adverse effect in a biological system (van Leeuwen, 1996)

Toxicity: Inherent property of an agent to cause an adverse biological effect (Organisation for Economic Cooperation and Development, 2002).

Toxic Factor obtained by taking the ratio equivalence of the POD (point of departure) of factor $(T E F)$ : the index compound to that of each of the other members in a group with a common mechanism of toxicity. Exposure to each chemical in the group is then multiplied by the appropriate TEF to express all exposures in terms of the index compound (Wilkinson et al., 2000).

Toxicodynamics: The process of interaction of chemical substances with target sites and the subsequent reactions leading to adverse effects (World Health Organization, 1994).
Toxicokinetics: The process of the uptake of potentially toxic substances by the body, the biotransformation they undergo, the distribution of the substances and their metabolites in the tissues and the elimination of the substances and their metabolites from the body. Both the amounts and concentrations of the substances and their metabolites are studied. The term has essentially the same meaning as pharmacokinetics, but the latter term should be restricted to the study of pharmaceutical substances (World Health Organization, 1994).

Upper level $(U L)$ :

The maximum level of total chronic daily intake of a nutrient (from all sources) judged to be unlikely to pose a risk of adverse health effects to humans (Scientific Committee on Food, 2000c).

Uncertainty: Imperfect knowledge concerning the present or future state of an organism, system or (sub) population under consideration (Organisation for Economic Co-operation and Development, 2002).

Uncertainty Synonymous with Safety Factor factor:

Xenobiotic: A chemical which is not a natural component of the organism, system or (sub) population exposed to it (Duffus, 2000). 